\title{
fluid flow in drying drops
}

Hanneke Gelderblom

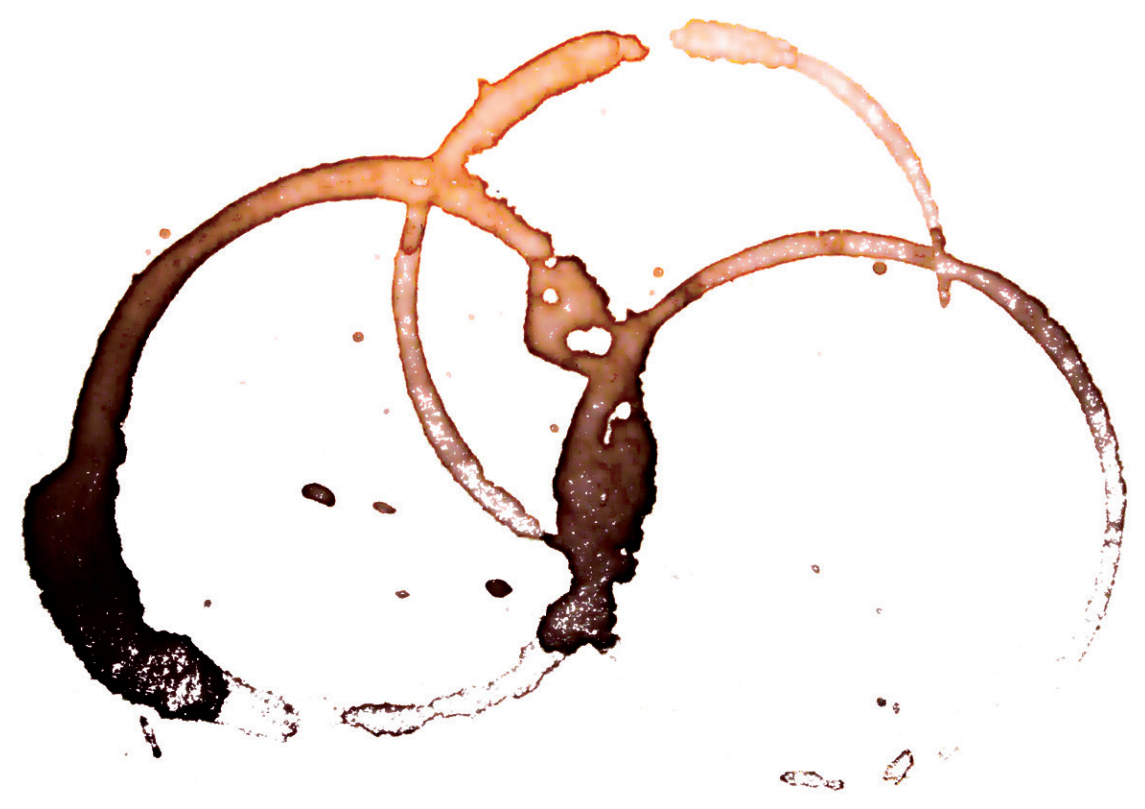


Fluid flow in drying drops

Hanneke Gelderblom 


\section{Samenstelling promotiecommissie:}

Prof. dr. ir. Leen van Wijngaarden (voorzitter) Universiteit Twente Prof. dr. rer. nat. Detlef Lohse (promotor) Universiteit Twente Dr. ir. Jacco H. Snoeijer (assistent-promotor) Universiteit Twente Dr. ir. Ramin Badie Prof. dr. ir. Jos P. H. Benschop Prof. dr. ir. Hans Hilgenkamp Prof. dr. ir. Frans N. van de Vosse ASML

Universiteit Twente \& ASML Universiteit Twente Technische Universiteit Eindhoven Prof. dr. Stephen K. Wilson University of Strathclyde

The work described in this thesis was carried out at the Physics of Fluids group of the Faculty of Science \& Technology of the University of Twente. It was financially supported by the Spinoza program of the "Nederlandse Organisatie voor Wetenschappelijk Onderzoek" (NWO).

Nederlandse titel:

Vloeistofstroming in opdrogende druppels

Publisher:

Hanneke Gelderblom, Physics of Fluids, University of Twente, P.O. Box 217, 7500 AE Enschede, The Netherlands pof.tnw.utwente.nl

Cover design: Xanthe van Haaften www.xanthevanhaaften.nl

Print: Gildeprint Drukkerijen, Enschede

(c) Hanneke Gelderblom, Enschede, The Netherlands, 2013

No part of this work may be reproduced by print photocopy or any other means without the permission in writing from the publisher ISBN: 978-90-365-3525-0

DOI: $10.3990 / 1.9789036535250$ 


\section{FLUID FLOW IN DRYING DROPS}

\section{PROEFSCHRIFT}

ter verkrijging van

de graad van doctor aan de Universiteit Twente,

op gezag van de rector magnificus,

Prof. dr. H. Brinksma,

volgens besluit van het College voor Promoties

in het openbaar te verdedigen

op vrijdag 19 april 2013 om 16.45 uur

door

Hanneke Gelderblom

geboren op 30 september 1985

te Eindhoven 
Dit proefschrift is goedgekeurd door de promotor:

Prof. dr. rer. nat. Detlef Lohse

en de assistent-promotor:

Dr. ir. Jacco H. Snoeijer 


\section{Contents}

1 Introduction 1

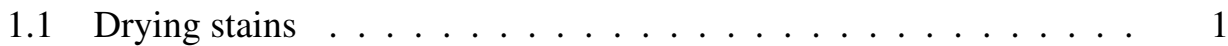

1.2 Physics of drying drops . . . . . . . . . . . . . . . . . . 3

1.3 Fluid flow in drying drops $\ldots \ldots \ldots \ldots$. . . . . . . . 7

1.4 Guide through the thesis $\ldots \ldots \ldots \ldots$

2 How water drops evaporate from a superhydrophobic substrate 15

2.1 Introduction . . . . . . . . . . . . . . . . . . . . . . . 16

2.2 Experimental methods . . . . . . . . . . . . . . . . . 18

2.3 Experimental results . . . . . . . . . . . . . . . . 20

2.4 Theory of drop evaporation . . . . . . . . . . . . . . . . 21

2.5 Comparison between theory and experiment . . . . . . . . . 24

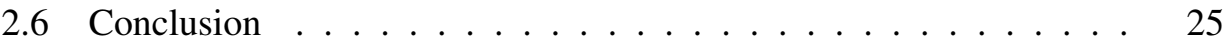

3 Stokes flow near the contact line of an evaporating drop 29

3.1 Introduction . . . . . . . . . . . . . . . . . 30

3.2 Corner solutions . . . . . . . . . . . . . . . . . . . . 31

3.3 Results . . . . . . . . . . . . . . . . . . . . . . . . . 39

3.4 Discussion . . . . . . . . . . . . . . . . 43

4 Stokes flow in a drop evaporating from a liquid subphase 51

4.1 Introduction . . . . . . . . . . . . . . 52

4.2 Problem formulation . . . . . . . . . . . . . . 54

4.3 Results . . . . . . . . . . . . . . . . . . . . 60

4.4 Discussion . . . . . . . . . . . . . . . . . 67

5 Order-to-disorder transition in ring-shaped colloidal stains 73

5.1 Introduction . . . . . . . . . . . . . . . . . 74

5.2 Analysis of the colloidal stain . . . . . . . . . . . . . . 74

5.3 Rush hour in the drying drop . . . . . . . . . . . . . . . . 76 
5.4 Order-to-disorder transition . . . . . . . . . . . . . . . . . . 79

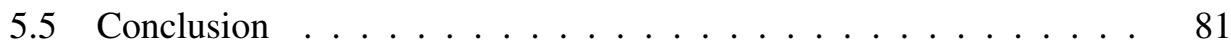

6 Building microscopic soccer balls with evaporating colloidal fakir drops 91

6.1 Introduction . . . . . . . . . . . . . . . . 92

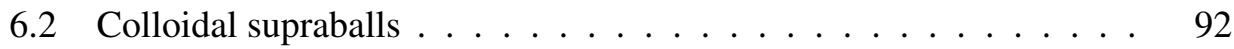

6.3 Drop evaporation . . . . . . . . . . . . . . . . . . . . . 94

6.4 Packing fraction of the supraballs . . . . . . . . . . . . . . . 96

6.5 Order-to-disorder transition . . . . . . . . . . . . . . . . . . 98

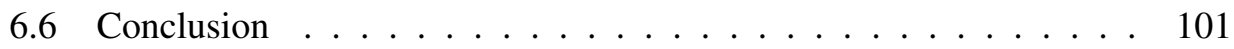

7 Oscillations of a gas pocket on a liquid-covered solid surface 105

7.1 Introduction . . . . . . . . . . . . . . . . . . 105

7.2 Problem formulation . . . . . . . . . . . . . . . . . 107

7.3 Potential flow . . . . . . . . . . . . . . . . . . . 110

7.4 Weak viscous effects . . . . . . . . . . . . . . . . . . 112

7.5 Unsteady Stokes flow . . . . . . . . . . . . . . . . . . . . . . 114

7.6 Numerical solution method . . . . . . . . . . . . . . . . . . . 115

7.7 Results . . . . . . . . . . . . . . . . . . . . . . . . 116

7.8 Conclusion . . . . . . . . . . . . . . . . 120

8 Summary and Outlook 129

$\begin{array}{ll}\text { Samenvatting } & 137\end{array}$

$\begin{array}{ll}\text { Acknowledgements } & 141\end{array}$

$\begin{array}{ll}\text { About the author } & 145\end{array}$ 


\section{1 \\ Introduction}

\subsection{Drying stains}

When you spill a drop of coffee or tea on a table and let it dry, it leaves behind a distinct stain (see Fig. 1.1). All the coffee particles, which were initially uniformly distributed over the drop, have collected into a dark ring at the edge of the drop. This so-called coffee-stain effect was first investigated by Robert Deegan and co-workers $[1,2]$. They discovered how particle transport occurs in drying drops where the edge of the drop, the contact line, is pinned to the underlying substrate, e.g. the table top. It turns out that a strong radially outward flow is generated inside such an evaporating drop. This flow drags the particles along towards the contact line, where the water evaporates while coffee particles are left behind and form the ring stain. The coffeestain effect is not specific for coffee and tea, but it can happen with any type of colloidal suspension of tiny particles such as ink, paint, salty water, and blood, as shown in Fig. 1.2.

Drying stains are not only encountered in daily life, they also appear in many industrial applications. In the coating and printing industry, the challenge is to prevent the formation of ring-shaped drying stains in order to obtain uniform coatings or homogeneously dried ink drops $[1,2,4]$. Ring-stain formation is also problematic in the analysis of DNA and proteins in microarrays: the inhomogeneous distribution of molecules in the dried spots leads to spurious changes in the signal intensity [5-7], as 


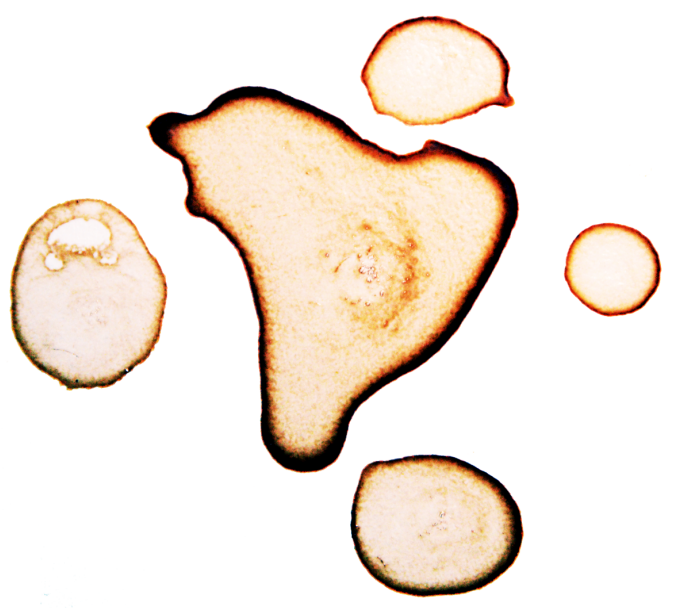

Figure 1.1: The coffee-stain effect demonstrated by tea stains on a desk: when the tea drop has dried, a dark ring of tea particles is left behind.
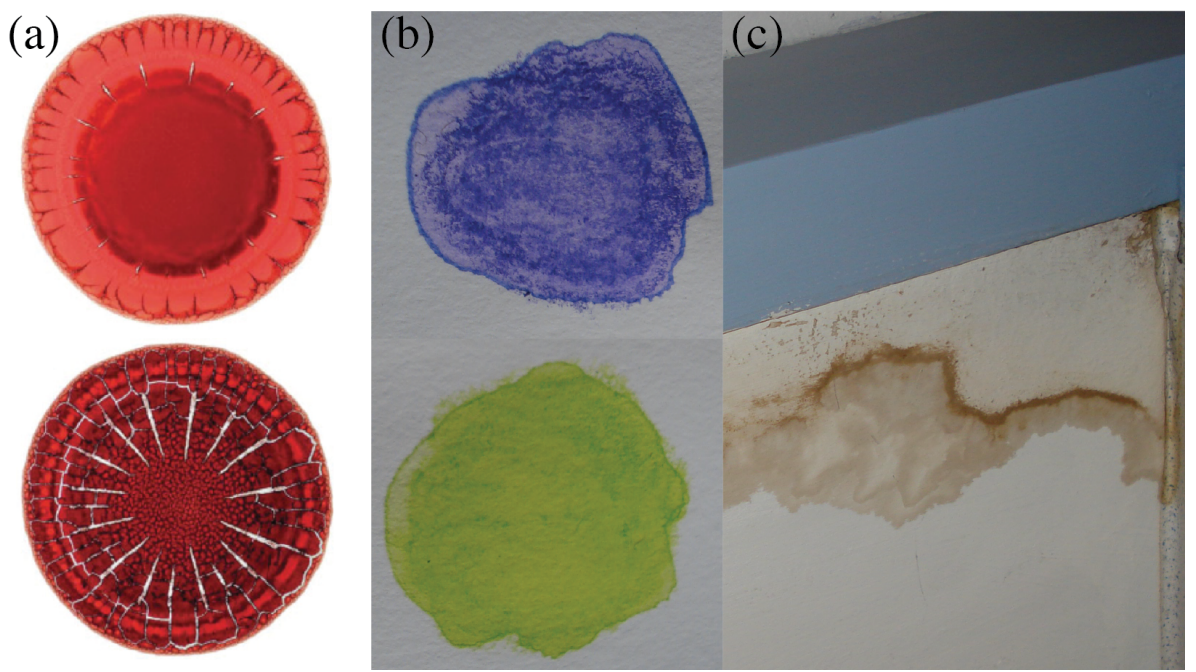

Figure 1.2: (a) Drying stains of blood drops (adapted from Brutin \& Sobac [3]), (b) stains formed by dried water colour paint, (c) pattern formation on a ceiling in the Molvense Erven, Nuenen (NL). 
(a)

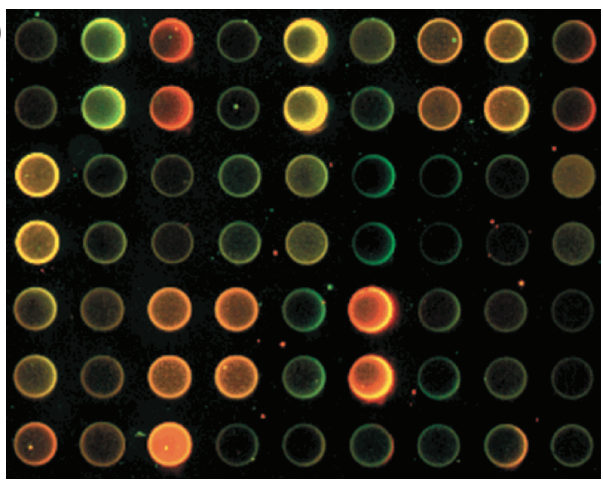

(b)

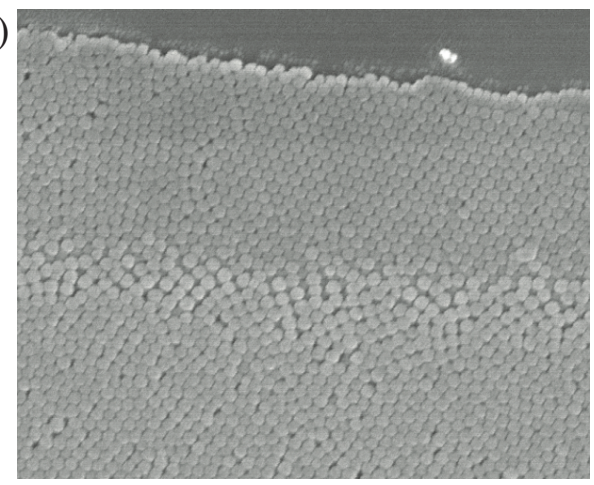

Figure 1.3: (a) Ring-stain formation in a DNA microarray leads to spurious changes in the signal intensity (adapted from Blossey \& Bosio [6]). (b) Stripe patterns of ordered silica particles formed by evaporation (adapted from Watanabe et al. [19]).

demonstrated in Fig. 1.3(a). Recently, it has been shown that this unwanted ring-stain formation can be suppressed by using non-spherical particles [8] or by shaking the drying drops with strong electric fields [9]. Stain formation by drop evaporation can however also be useful: it provides a way to self-assemble small particles into a fine pattern on a scale where direct manipulation is impossible [10-12], see Fig. 1.3(b). In this way, evaporation is used to generate self-assembled monolayers of tiny particles [13] and colloidal crystals [14-18].

Hence, pattern formation by evaporating drops can be disadvantageous when it leads to unwanted ring-stains, but it can also be used to self-assemble particles into tiny structures. In order to make use of the coffee-stain effect, one needs to be able to predict and control the stain formation. At present, there is no way of predicting beforehand the structure of the stain that will form, due to a lack of understanding of the basic physical mechanisms that play a role during drop evaporation. In particular, to know how an evaporating drop can shape a pattern, the physics of drying drops and their internal fluid flow has to be understood. The purpose of the work described in this thesis is therefore to address the fundamental physics of drop evaporation, evaporation-driven flow inside the drying drop, and the subsequent particle transport.

\subsection{Physics of drying drops}

We first discuss the basic physical mechanism of evaporation. When a liquid drop is deposited onto a substrate, such as a coffee drop on a table top, it attains a spherical- 

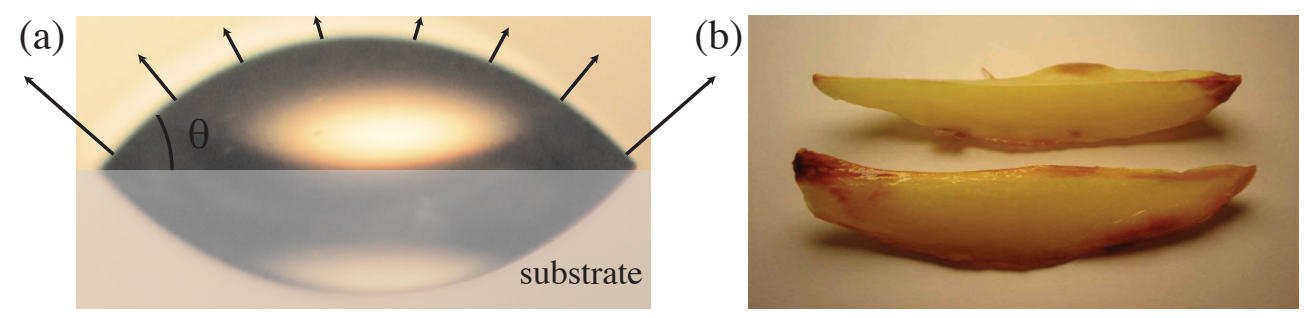

Figure 1.4: (a) A water drop on a glass slide with a contact angle $\theta$ of about $45^{\circ}$. The reflection of the drop in the substrate illustrates the sharp edge that appears when $\theta<90^{\circ}$. The arrows indicate the non-uniform evaporative flux from the surface of the drop. (b) Rapid dehydration from sharp edges causes potato wedges to burn at the edges first (adapted from Bocquet [20]).

cap shape with a contact angle $\theta$ with the substrate. Fig. 1.4(a) shows a picture of such a macroscopic water drop on a glass slide. Evaporation causes the volume of the sessile drop to decrease, until eventually a dry spot remains. There are three physical mechanisms that can influence the drying rate of a sessile drop. The first mechanism is the phase change from liquid to vapour: the rate at which molecules can cross the liquid-air interface $[21,22]$. The second mechanism is the transport of the vapour molecules away from the drop in the surrounding air. This transport can be purely diffusive [1,23, 24], or a combination of diffusive and free convective transport [25]. The third rate-limiting step is the evaporative cooling and heat transfer to the liquidair interface [22, 26-28]. In this thesis, we focus on macroscopic sessile water drops under atmospheric conditions. In this case, the evaporation rate is usually determined by the diffusive transport of water vapour in the surrounding air [1, 2, 21, 23, 24].

\subsubsection{Singular evaporative flux}

Under atmospheric conditions, liquid molecules rapidly cross the liquid-air interface, such that the air just above the drop becomes saturated with vapour. If the surrounding air far from the drop surface is not saturated, the vapour diffuses outward. The rate at which the drop volume decreases is determined by this diffusive transport of vapour away from the drop. Due to evaporation, the liquid-air interface is not stationary, but slowly moves as the volume of the drop decreases. The time-dependent vapour concentration $c$ in the air surrounding the drop is governed by the unsteady diffusion equation

$$
\frac{\partial c}{\partial t}=D \nabla^{2} c
$$


where $D$ is the diffusion constant for vapour in air. However, the evaporation-induced motion of the drop surface is typically much slower than the diffusion time for the vapour transport, so that the time-dependent term in (1.1) can be neglected and the quasi-steady concentration field is given by

$$
\nabla^{2} c=0
$$

The strength of the evaporative flux $J$ from the drop surface is determined by the gradient in the vapour concentration according to

$$
\mathbf{J}=-D \nabla c .
$$

To find the evaporative flux from the vapour concentration field, (1.2) is solved subject to the boundary conditions that the vapour concentration equals the saturated vapour concentration $c_{s a t}$ just above the drop surface, and $c_{\infty}$ far away. When $c_{\infty}<c_{s a t}$, a diffusive outflux arises and the drop evaporates. A third boundary condition is that the substrate on which the drop is deposited is impermeable to the vapour. The evaporative flux is found from (1.3). It turns out that this evaporative flux is not uniform, but stronger at the pointy edge of the drop if the contact angle is below $90^{\circ}$, as indicated by the arrows in Fig. 1.4(a). To understand the origin of this non-uniform evaporative flux, we focus on the region close to the contact line of the drop. Mathematically the impermeability condition is equivalent to mirror-imaging the drop, as shown in Fig. 1.4(a). From the picture of the drop and its reflection it is clear that the drop has a pointy, singular shape near the contact line. The shape of the drop near the contact line can be approximated by a wedge geometry and a self-similar solution to the evaporative flux can be obtained, which scales as [1]

$$
J \sim \frac{D \Delta c}{R}\left(\frac{\rho}{R}\right)^{\lambda(\theta)-1},
$$

with $\Delta c=c_{s a t}-c_{\infty}, R$ the drop base radius, $\rho$ the radial distance to the contact line, and

$$
\lambda(\theta)=\frac{\pi}{2(\pi-\theta)} .
$$

Hence, for contact angles below $90^{\circ}$ (when the edge is sharp) $\lambda<1$, and the evaporative flux diverges as the contact line is approached. The sharper the edge of the drop, the stronger this divergence. For very flat drops $\left(\theta \ll 90^{\circ}\right)$ a square-root divergence of the evaporative flux is found. The mathematical problem of finding the vapour concentration field around a sharp edge has an electrostatic equivalent: the electrostatic potential around a charged conductor. At the sharp tip of e.g. a lightning rod all equipotential lines come close together, which leads to a divergence of 
the electric field and an accumulation of charge on the tip of the rod. Similarly, all the iso-concentration lines come close together at the contact line of an evaporating drop with a contact angle below $90^{\circ}$, so that the evaporative flux diverges. This rapid dehydration from sharp edges is also the reason why potato wedges in the oven burn at the edges first, as shown in Fig. 1.4(b) [20].

\subsubsection{Lifetime of a liquid drop}

To find the lifetime of a sessile drop, the evaporative flux from the entire drop surface has to be known. However, without knowing the exact expression for the evaporative flux, one can estimate the rate at which the drop volume decreases from dimensional arguments. The typical drop volume $V$ scales as $V \sim R^{3}$. The time scale on which vapour transport occurs is the diffusion time $t_{d}=R^{2} / D$. Hence, the rate of volume change scales as

$$
\frac{d V}{d t} \sim-\frac{R^{3}}{t_{d}}=-D R,
$$

which implies that the rate of volume change is proportional to the perimeter of the drop, and not to its surface area.

The way the liquid-air interface moves as the drop volume decreases depends on the mode along which the evaporation takes place. Drop evaporation can occur along two basic modes [2, 29-31]. The first mode is the constant contact-angle mode, in which the contact line is free to move and the contact area of the drop with the substrate decreases, see Fig. 1.5(a) . The second mode is the constant contact-area mode, in which the contact line is pinned and the contact angle of the drop slowly decreases, as shown in Fig. 1.5(b). If the contact line is free to move, we can obtain the time-dependent drop radius by using that for a constant contact angle

$$
\frac{d V}{d t} \sim R^{2} \frac{d R}{d t},
$$

owing to the self-similarity of the spherical cap in case the contact angle does not change. Combining (1.6) and (1.7) we find that the drop radius

$$
R(t) \sim \sqrt{D\left(t_{f}-t\right)},
$$

with $t_{f}$ the total evaporation time. This means that the drop radius very rapidly shrinks during the final stages of evaporation [25, 32].

In case the contact line is pinned, the drop radius is constant in time and the rate of mass loss at each instant is solely dependent on the contact angle. The expressions for the rate of mass loss and the evolution of the contact angle have to be obtained from 
(a)

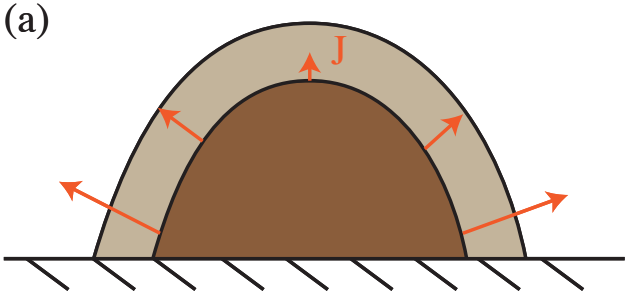

(b)

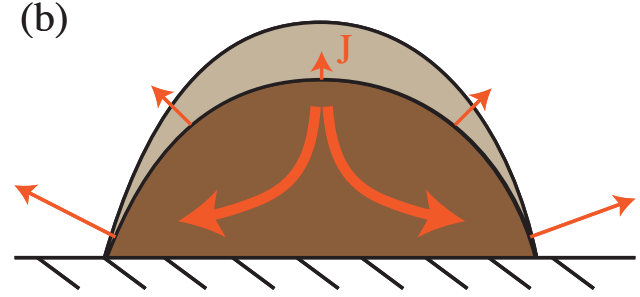

Figure 1.5: (a) A drop evaporating in constant contact-angle mode: the contact area decreases while the contact angle remains constant. The flow in the drop has an outward contribution that comes from the evaporative flux $J$ from the drop surface and an inward contribution that comes from the receding motion of the contact line. (b) A drop evaporating in constant contact-area mode: the contact angle decreases while the contact area remains constant. The evaporative flux from the surface of the drop generates a radially outward flow.

the drop geometry and the exact solution to the evaporative flux from the drop surface. In the limit of small contact angles $\left(\theta \ll 90^{\circ}\right)$ simple analytical expressions for this evaporative flux can be found [2]. Finding the evaporative flux from a spherical-cap shaped drop of arbitrary contact angle however is more complicated and involves the use of non-trivial special functions [24, 33]. Until now, the validity of these diffusionbased evaporation models has only been investigated for drops with contact angles below $90^{\circ}[2,23]$. In this thesis, we will study drops on superhydrophobic substrates, where the initial contact angle $\gg 90^{\circ}$.

\subsection{Fluid flow in drying drops}

In drying coffee drops, the evaporative flux from the drop surface drives a radially outward flow inside the drop which transports the coffee particles. When the contact line of the drop is pinned, as in Fig. 1.5(b), the liquid that evaporates from the contact line region must be replenished by liquid from the interior of the drop $[1,2]$. A capillary mechanism drives the flow: in order to maintain the drop's spherical-cap shape dictated by surface tension, a compensating flow is required to refill the liquid that evaporates from the pinned contact line. Pinning of the contact line occurs by pre-existing roughness of the substrate. On top of this, suspended particles that arrive at the contact line due to the coffee-stain effect re-inforce the pinning, and thereby generate a self-pinning mechanism [34].

The amount of flow inside an evaporating drop can be calculated from mass con- 


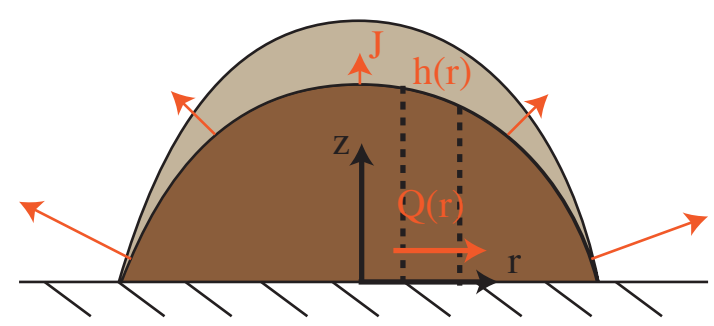

Figure 1.6: Schematic representation of an axisymmetric drop that is evaporating, described in a cylindrical coordinate system $(r, z)$. The evaporative flux $J$ from the drop surface drives a flow $Q(r)$ inside the drop with height profile $h(r)$. The dashed lines indicate the control volume of width $d r$.

servation once the evaporative flux (1.3) is known. To illustrate this, we consider an axisymmetric drop with a pinned contact line, described in a cylindrical coordinate system $(r, z)$, see Fig. 1.6. Inside this drop, we define an infinitesimally small control volume of width $d r$, located at a distance $r$ from the drop center. Mass conservation requires that the rate of change of the amount of liquid inside this control volume equals the net inflow of liquid into the control volume, minus the amount of liquid that has evaporated from the drop surface. This can be expressed as [2]

$$
\frac{\partial h}{\partial t}=-\frac{1}{r} \frac{\partial}{\partial r} Q-\frac{1}{\rho_{\ell}} J,
$$

where $h(r, t)$ is the local drop height, $t$ the time, $Q$ the volume flow and $\rho_{\ell}$ the liquid density. Hence, the decrease in drop height of a volume element equals the evaporative flux through the free surface of the element plus the net outflow to the neighbouring elements.

In the present context, we use the mass conservation (1.9) to compute the liquid flow $Q$ inside the drop. Namely, $\partial h / \partial t$ can be obtained by combining the total rate of volume change (as explained in the previous paragraph), and the assumption that the drop shape remains that of a spherical cap. The evaporative flux is known from the vapour concentration field (1.3), and by integration over the drop surface the total rate of volume change can be found. Hence, once the evaporative flux is known, (1.9) is an equation for the flow inside the drop. For relatively flat drops, with a contact angle $\theta \ll 90^{\circ}$, the expressions for the drop volume and evaporative flux are simple and the flow can be obtained from (1.9) by integration [1]. From drops of arbitrary contact angle however, this is much more complicated [24], and closed-form expressions cannot be obtained.

Although the volume flow inside the drop can be found from mass conservation, 
the exact velocity profile inside the drop remains unknown. In the small contactangle regime, the lubrication approximation is often used to describe the velocity field inside the drop [35, 36]. However, even for relatively flat drops $\left(\theta \ll 90^{\circ}\right)$ the validity of this approximation near the contact line, where the evaporative flux diverges, has been questioned [37]. On top of that, Marangoni stresses originating from temperature differences in the drop could lead to additional recirculation near the contact line [38-40]. Furthermore, if the contact line is not pinned but free to move, there will be an additional contribution to the velocity field that comes from the motion of the contact line $[35,36]$. Hence, it is not yet known what the velocity field inside the drop looks like, how it evolves over time and how this affects the dynamics of the stain formation.

\subsection{Guide through the thesis}

In this thesis we investigate the evaporation of colloidal dispersion drops in order to understand how evaporation and flow control the particle deposition. As a simple model system, we use macroscopic water drops that contain spherical polystyrene particles, and study the evaporation of these drops under atmospheric conditions.

In Chapter 2 we investigate what determines the evaporation rate of a water drop on a superhydrophobic substrate. We show that the evaporation rate of such a drop is indeed entirely determined by diffusive transport of the water vapour in the surrounding air and occurs in a universal way for all drop sizes and arbitrary contact angles. In Chapter 3 we focus on the inside of the drop: how does the internal flow look as the drop evaporates from a flat solid substrate? We derive analytical solutions to the velocity field close to the contact line of the drop and show that the nature of the flow changes in a spectacular way with the contact angle. We extend this concept to drops evaporating from a (non-volatile) soft or liquid substrate in Chapter 4. It turns out that the evaporation of such a drop also generates a flow pattern in the non-volatile liquid subphase. Again, the nature of the flow near the contact line strongly depends on the contact angles between the two liquids and the surrounding air.

Once the flow inside the evaporating drop is known, we can predict what type of stain will be obtained when we add colloidal particles. In Chapter 5 we study the evaporation of colloidal dispersion drops with pinned contact lines, and obtain detailed information on the dynamics of the ring-stain formation. We show that from the deposition dynamics, we can predict and control the pattern that forms. Pattern formation by drops with moving contact lines is discussed in Chapter 6. We let drops evaporate from pillared superhydrophobic substrates on which there is no pinning of the contact line. These pillars are shaped in such a way that the drop remains in the 
fakir state on top of the pillars during evaporation. In that case the end result is not a deposited ring stain, but an ordered, soccer-ball-like three-dimensional object.

In Chapter 7 we study the inverse of a liquid drop in air: an air bubble in liquid. We develop a model to study the response of the liquid-air interface to a pressure pulse, and calculate the resonance frequency and interface shape of the insonated bubble.

\section{References}

[1] R. D. Deegan, O. Bakajin, T. F. Dupont, G. Huber, S. R. Nagel, and T. A. Witten, "Capillary flow as the cause of ring stains from dried liquid drops", Nature 389, 827-828 (1997).

[2] R. D. Deegan, O. Bakajin, T. F. Dupont, G. Huber, S. R. Nagel, and T. A. Witten, "Contact line deposits in an evaporating drop", Phys. Rev. E 62, 756765 (2000).

[3] D. Brutin, B. Sobac, B. Loquet, and J. Sampol, "Pattern formation in drying drops of blood", J. Fluid Mech. 667, 85-95 (2011).

[4] J. Perelaer, P. J. Smith, C. E. Hendriks, A. M. J. van den berg, and U. S. Schubert, "The preferential deposition of silica micro-particles at the boundary of inkjet printed droplets", Soft Matter 4, 1072 (2008).

[5] Y. Kim, G. Hurst, M. Doktycz, and M. Buchanan, "Improving spot homogeneity by using polymer substrates in matrix-assisted laser desorption/ionization mass spectrometry of oligonucleotides", Anal. Chem. 73, 2617-2624 (2001).

[6] R. Blossey and A. Bosio, "Contact line deposits on cDNA microarrays: a twinspot effect”, Langmuir 18, 2952-2954 (2002).

[7] G. McHale, "Surface free energy and microarray deposition technology", Analyst 132, 192-195 (2007).

[8] P. J. Yunker, T. Still, M. A. Lohr, and A. G. Yodh, "Suppression of the coffeering effect by shape-dependent capillary interactions", Nature 476, 308-311 (2011).

[9] H. B. Eral, D. M. Augustine, M. H. G. Duits, and F. Mugele, "Suppressing the coffee stain effect: how to control colloidal self-assembly in evaporating drops using electrowetting", Soft Matter 7, 1-5 (2011). 
[10] J. Boneberg, F. Burmeister, C. Schäfle, P. Leiderer, D. Reim, A. Fery, and S. Herminghaus, "The formation of nano-dot and nano-ring structures in colloidal monolayer lithography", Langmuir 13, 7080-7084 (1997).

[11] L. Meng, H. Wei, A. Nagel, B. J. Wiley, L. E. Scriven, and D. J. Norris, “The role of thickness transitions in convective assembly", Nano Lett. 6, 2249-2253 (2006).

[12] M. Layani, M. Gruchko, O. Milo, I. Balberg, D. Azulay, and S. Magdassi, "Transparent conductive coatings by printing coffee ring arrays obtained at room temperature", ACS Nano 3, 3537-3542 (2009).

[13] T. P. Bigioni, X. M. Lin, T. T. Nguyen, E. I. Corwin, T. A. Witten, and H. M. Jaeger, "Kinetically driven self assembly of highly ordered nanoparticle monolayers", Nat. Mater. 5, 265-270 (2006).

[14] K. P. Velikov, C. G. Christova, R. P. A. Dullens, and A. van Blaaderen, "Layerby-layer growth of binary colloidal crystals", Science 296, $106-109$ (2002).

[15] E. Dufresne, E. Corwin, N. A. Greenblatt, J. Ashmore, D. Wang, A. Dinsmore, J. Cheng, X. Xie, J. Hutchinson, and D. Weitz, "Flow and fracture in drying nanoparticle suspensions", Phys. Rev. Lett. 91, 224501 (2003).

[16] E. Kumacheva, P. Garstecki, H. K. Wu, and G. M. Whitesides, "Twodimensional colloid crystals obtained by coupling of flow and confinement", Phys. Rev. Lett. 91, 128301 (2003).

[17] M. Abkarian, J. Nunes, and H. A. Stone, "Colloidal crystallization and banding in a cylindrical geometry”, J. Am. Chem. Soc. 126, 5978-5979 (2004).

[18] F. Fan and K. J. Stebe, "Assembly of colloidal particles by evaporation on surfaces with patterned hydrophobicity", Langmuir 20, 3062-3067 (2004).

[19] S. Watanabe, K. Inukai, S. Mizuta, and M. Miyahara, "Mechanism for stripe pattern formation on hydrophilic surfaces by using convective self-assembly", Langmuir 25, 7287-7295 (2009).

[20] L. Bocquet, “Tasting edge effects”, Am. J. Phys. 75, 148 (2007).

[21] A. M. Cazabat and G. Guéna, "Evaporation of macroscopic sessile droplets", Soft Matter 6, 2591-2612 (2010). 
[22] N. Murisic and L. Kondic, "On evaporation of sessile drops with moving contact lines”, J. Fluid Mech. 679, 219-246 (2011).

[23] H. Hu and R. G. Larson, "Evaporation of a sessile droplet on a substrate", J. Phys. Chem. B 106, 1334-1344 (2002).

[24] Y. O. Popov, "Evaporative deposition patterns: Spatial dimensions of the deposit”, Phys. Rev. E 71, 036313 (2005).

[25] N. Shahidzadeh-Bonn, S. Rafai, A. Azouni, and D. Bonn, "Evaporating droplets”, J. Fluid Mech. 549, 307-313 (2006).

[26] S. David, K. Sefiane, and L. Tadrist, "Experimental investigation of the effect of thermal properties of the substrate in the wetting and evaporation of sessile drops", Colloids Surf. A 298, 108-114 (2007).

[27] G. J. Dunn, S. K. Wilson, B. R. Duffy, S. David, and K. Sefiane, "A mathematical model for the evaporation of a thin sessile liquid droplet: Comparison between experiment and theory", Colloids Surf. A 323, 50-55 (2008).

[28] G. J. Dunn, S. K. Wilson, B. R. Duffy, S. David, and K. Sefiane, "The strong influence of substrate conductivity on droplet evaporation", J. Fluid Mech. 623, 329-351 (2009).

[29] R. G. Picknett and R. Bexon, "The evaporation of sessile or pendant drops in still air”, J. Colloid Interface Sci. 61, 336-350 (1977).

[30] K. S. Birdi, D. T. Vu, and A. Winter, "A study of the evaporation rates of small water drops placed on a solid surface”, J. Phys. Chem. 93, 3702-3703 (1989).

[31] H. Y. Erbil, G. McHale, and M. I. Newton, "Drop evaporation on solid surfaces: Constant contact angle mode", Langmuir 18, 2636-2641 (2002).

[32] G. Guéna, C. Poulard, and A. M. Cazabat, "The leading edge of evaporating droplets”, J. Colloid Interface Sci. 312, 164-171 (2007).

[33] N. N. Lebedev, Special functions and their applications (Prentice-Hall) (1965).

[34] R. D. Deegan, "Pattern formation in drying drops", Phys. Rev. E 61, 475-485 (2000).

[35] G. Berteloot, C. T. Pham, A. Daerr, F. Lequeux, and L. Limat, "Evaporationinduced flow near a contact line: consequences on coating and contact angle", Europhys. Lett. 83, 14003 (2008). 
[36] J. Eggers and L. M. Pismen, "Nonlocal description of evaporating drops", Phys. Fluids 22, 112101 (2010).

[37] H. Hu and R. G. Larson, "Analysis of the microfluid flow in an evaporating sessile droplet”, Langmuir 21, 3963-3971 (2005).

[38] H. Bodiguel and J. Leng, "Imaging the drying of a colloidal suspension", Soft Matter 6, 5451-5460 (2010).

[39] H. Hu and R. Larson, "Marangoni effect reverses coffee-ring depositions", J. Phys. Chem. B 110, 7090-7094 (2006).

[40] W. D. Ristenpart, P. G. Kim, C. Domingues, J. Wan, and H. A. Stone, "Influence of substrate conductivity on circulation reversal in evaporating drops", Phys. Rev. Lett. 99, 234502 (2007). 


\section{2 \\ How water drops evaporate from a superhydrophobic substrate ${ }^{* \dagger}$}

In this chapter, the evaporation of water drops from a superhydrophobic substrate on which the contact line is pinned is investigated. While previous studies mainly focused on drops with contact angles smaller than $90^{\circ}$, here we analyse almost the full range of possible contact angles $\left(10^{\circ}-150^{\circ}\right)$. The greater contact angles and pinned contact lines can be achieved by the use of superhydrophobic Carbon Nanofiber substrates. The time-evolutions of the contact angle and the drop mass are examined. The experimental data is in good quantitative agreement with the model presented by Popov [Physical Review E 71, 036313 (2005)], demonstrating that the evaporation process is quasi-static, diffusion-driven, and that thermal effects play no role. Furthermore, we show that the experimental data for the evolution of both the contact angle and the drop mass can be collapsed onto one respective universal curve for all drop sizes and initial contact angles.

*Published as: Hanneke Gelderblom, Álvaro G. Marín, Hrudya Nair, Arie van Houselt, Leon Lefferts, Jacco H. Snoeijer, and Detlef Lohse, "How water droplets evaporate on a superhydrophobic substrate", Phys. Rev. E 83, 026306 (2011).

${ }^{\dagger}$ The experimental work is performed by Álvaro G. Marín and Hrudya Nair. 


\subsection{Introduction}

Evaporation of sessile drops with small contact angles $\left(<90^{\circ}\right)$ has been studied extensively. Several evaporation modes have been explored: the constant contact-angle mode $[1,2]$, in which the contact area of the drop on the substrate vanishes; the constant contact-area mode [1,3-6], in which the contact angle vanishes; and the combination of both modes $[1,7,8]$. A thorough understanding of drop evaporation is of vital importance for examining the drying rate $[1,3,6,7,9-11]$, the flow patterns observed inside drying drops [12-14], and the residual deposits $[5,15,16]$.

In early modelling of evaporating drops $[3,4,7,17]$, the evaporative flux was assumed to be uniform in the radial direction, as it is for evaporation from a sphere. However, in his study of contact-line deposits, Deegan [5] argued that the evaporative flux from a sessile drop with a spherical-cap shape is generally not uniform, but diverges near the edge of the drop for contact angles smaller than $90^{\circ}$ (see Chapter 1). Hu and Larson [6] later used a numerical model to find an expression for the rate of mass loss from a drop in terms of its contact angle, taking this divergence into account. Their model applies to contact angles smaller than $90^{\circ}$.

For larger contact angles, few theoretical descriptions exist for diffusion around a spherical-cap drop. In [1], the rate of mass loss was expressed in terms of a series solution, which can be approximated in both the small and the large contact angle regimes. Popov [16] described an analytical solution for the rate of mass loss in terms of the contact angle that applies to the full range of contact angles. However, this model has never been validated against experimental data in the large contactangle regime.

Apart from the diffusive spreading of water vapour described by the models mentioned above, there are other factors that may influence the evaporation rate (see e.g. [18] for an overview). Firstly, the evaporation models discussed assume a stationary contact line. When the contact line is moving, dynamic effects may complicate the problem, for both the vapour concentration outside and the viscous flow inside the drop [8]. Secondly, evaporative cooling of the drop can reduce the evaporation rate [9-11] and the resulting temperature gradients on the drop surface can induce a Marangoni flow [13, 14]. Finally, in addition to the diffusion of water vapour, free convective transport may play a role, increasing the evaporation rate [11, 19]. However, the influence of these factors on the evaporation rate still has to be confirmed experimentally.

In this chapter, we describe our investigation into the evaporation of water drops from Carbon Nanofibre (CNF) substrates; see Fig. 2.1. These substrates belong to the family of ordered carbonaceous structures: the graphitic planes are oriented under an angle to the central axis. CNF substrates can exhibit superhydrophobicity [20]. The 


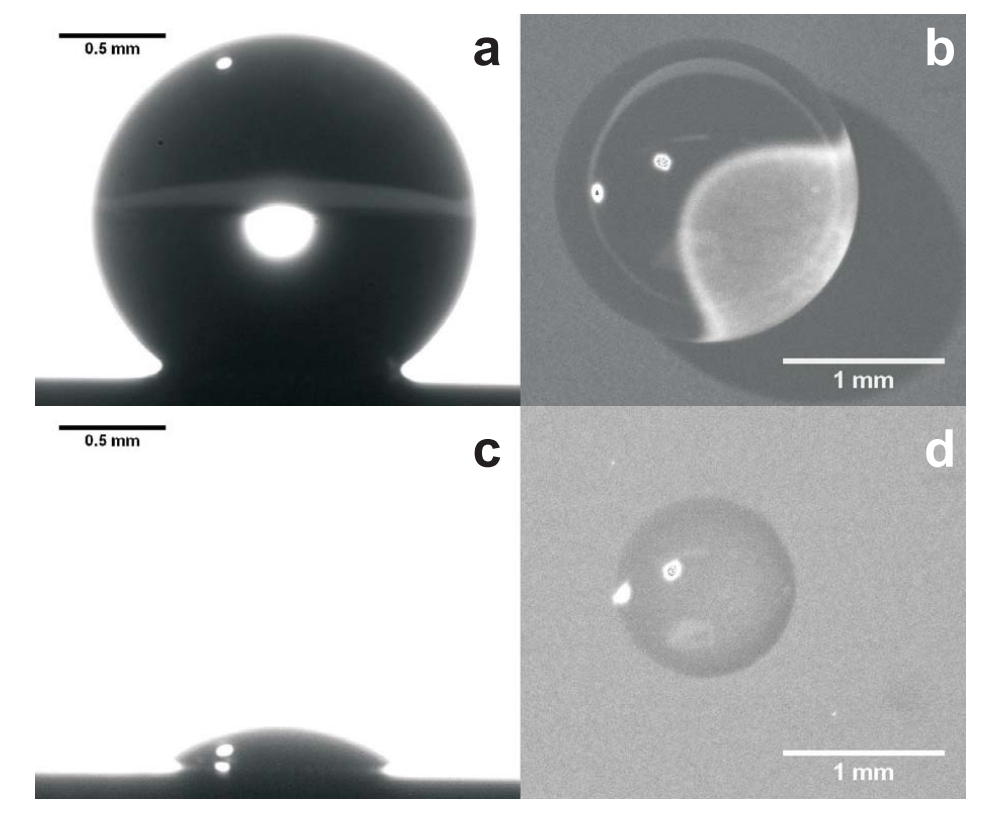

Figure 2.1: Side view (a) and top view (b) of a $8 \mu \mathrm{l}$ drop on a CNF substrate in the initial moments. (c) and (d): the same drop in the last moments before being completely evaporated. Note that the contact line remains perfectly circular and completely pinned until almost the end of the process.

samples used here have contact angles with water ranging from $150^{\circ}$ up to $170^{\circ}$. On superhydrophobic substrates, all evaporation modes can occur; the constant contactangle mode is mostly observed when the contact angle hysteresis is low, the constant contact-area mode when the hysteresis is high $[21,22]$. On our CNF substrates, the contact line remains pinned throughout nearly the entire experiment, hence evaporation takes place in the constant contact-area mode. Therefore, we can study evaporating drops in almost the full range of possible contact angles $\left(0^{\circ}-150^{\circ}\right)$. The rate of mass loss and contact-angle evolution over time are obtained experimentally for various drop sizes. We show that the evaporation dynamics is accurately described by the diffusion-based model of Popov [16], suggesting that thermal and free-convection effects are unimportant in our experiment. In addition, we show that the evolutions of the drop mass and contact angle can be described by a universal relation, that is, independent of the drop size and initial contact angle.

In Section 2.2, the experimental set-up and preparation of the CNF substrates are 


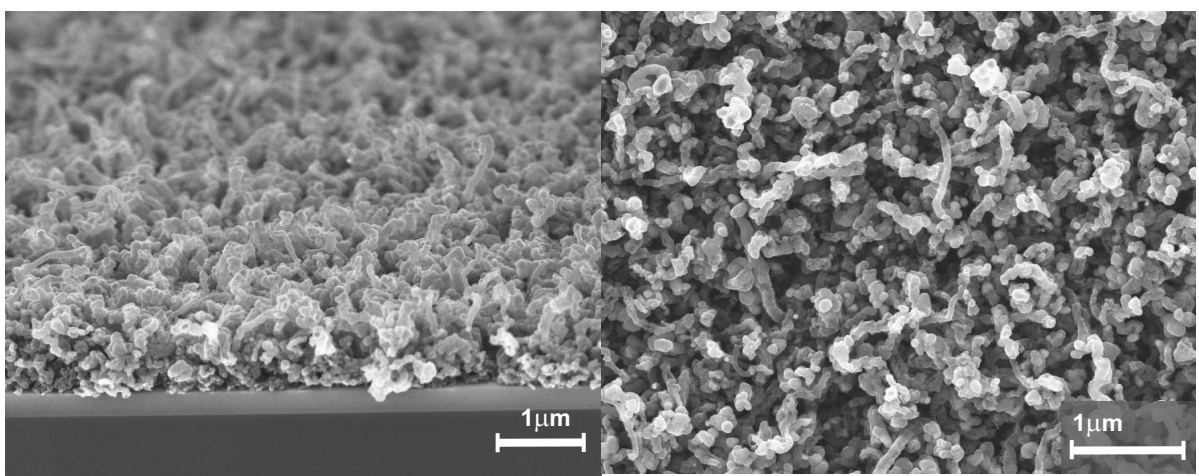

Figure 2.2: SEM images of the Carbon Nanofibres (CNFs) used as superhydrophobic substrates. Tilted side view (left) and augmented top view (right).

described. The experimental results are discussed in Section 2.3. The theoretical model for drop evaporation adopted from Popov [16] is briefly described in Section 2.4. Finally, in Section 2.5, it is shown that the theoretical results are in good quantitative agreement with the experimental data.

\subsection{Experimental methods}

\subsubsection{Preparation of the CNF substrates}

The drops were left evaporating in an empty room ${ }^{\ddagger}$ at a constant temperature of $23^{\circ}$ and a humidity of $30 \%$ over Carbon Nanofibre substrates; see Fig. 2.2. Carbon nanofibres were grown on oxidized silicon substrates using a $\mathrm{Ni}$ thin film as catalyst. A $250 \mathrm{~nm}$ thick $\mathrm{SiO}_{2}$ layer was grown on $p$-type $\mathrm{Si}(001)$ via wet oxidation. On top of this oxide layer $10 \mathrm{~nm}$ Ta was deposited, followed by a $25 \mathrm{~nm}$ thick Ni layer. The samples were pretreated prior to the CNF synthesis in a quartz reactor. The substrates were placed on a flat quartz boat placed centrally inside a quartz reactor, and the temperature was increased at a rate of $5^{\circ} \mathrm{C} \mathrm{min}^{-1}$ from room temperature up to $500^{\circ} \mathrm{C}$ in a $\mathrm{N}_{2}(99.999 \%$, Indugas) atmosphere. During this pretreatment step, the samples were subjected to $20 \mathrm{vol} . \%$ of $\mathrm{H}_{2}$ in $\mathrm{N}_{2}$ at a total flow rate of $50 \mathrm{ml}$ $\min ^{-1}$ at $500{ }^{\circ} \mathrm{C}$ for 2 hours; then the temperature was increased up to $635^{\circ} \mathrm{C}$. At 635 ${ }^{\circ} \mathrm{C}, 25$ vol.\% ethylene (99.95\% Praxair) in $\mathrm{N}_{2}$ was passed through the reactor for 1 hour, while 6.25 vol. $\% \mathrm{H}_{2}(99.999 \%$, Indugas) was added for the first minutes of the

\footnotetext{
${ }^{\ddagger}$ No human heat sources were present.
} 
reaction time. After the reaction time, the substrates were cooled down in $\mathrm{N}_{2}$ at a rate of $10^{\circ} \mathrm{C} \mathrm{min}{ }^{-1}$ until room temperature was reached. The CNF samples were used without further functionalization.

\subsubsection{Measurement of drop evaporation}

To analyse the evaporation of drops on CNF substrates, the drops were observed during their total evaporation time and photographed at $1 \mathrm{~s}$ time intervals. Two synchronized cameras (Lumenera Lm135, 1392 x 1040 pixels) were used for this purpose, one taking side-view images and another one taking top-view images; see Fig. 2.1. Side-view images allowed us to compute volume (mass), contact angle, area, drop radius, mass loss, and spreading velocity at every instant. The image analysis was performed using a custom-made MATLAB code in which the detected drop profile was fitted to an ellipse. The drops considered in this study are much smaller than the capillary length (which is $2.7 \mathrm{~mm}$ for a water drop [18]), hence we can neglect flattening of the drops by gravity. Nevertheless, we used an elliptical rather than a spherical fitting. The elliptical fit allowed us to use three fitting parameters (two semi axes and the angle of the ellipse with the horizontal plane) instead of only one (drop radius), thereby increasing the precision of the determination of the volume and contact angle of the drops. The ellipticity of the drops, defined as the ratio between both semi axes, was always below $7 \%$.

The contact line of the drops was detected automatically; the contact angles were then measured by finding the tangent of the ellipse at the contact line. The error in the determination of the contact angle, based on the quality of the fits, was found to be below $1 \%$. The volume of the drop was obtained by calculating the ellipse area above the contact line and assuming rotational symmetry with respect to the vertical axis, with an error below $10 \%$. The rate of mass loss was computed applying a fourth-order finite differentiation of the ellipse volume over time.

Top-view images were used to obtain qualitative information on the stability and circular symmetry of the contact line; using this information, we rejected those few experiments in which the contact line had a highly irregular shape.

Due to the chaotic three-dimensional distribution of the nanofibres, the way the liquid wets the structure is more complex than for ordered superhydrophobic microstructures [23, 24], for which two wetting states can be defined: the Cassie-Baxter state, in which the contact of the liquid with the substrate is minimum, and the Wenzel state, in which the contact is maximum. In our case, it is assumed that the liquid remains in a mixed state and that the transitions from one intermediate state to another are sufficiently smooth to be undetectable. Therefore, we will not use this terminology in this chapter. 

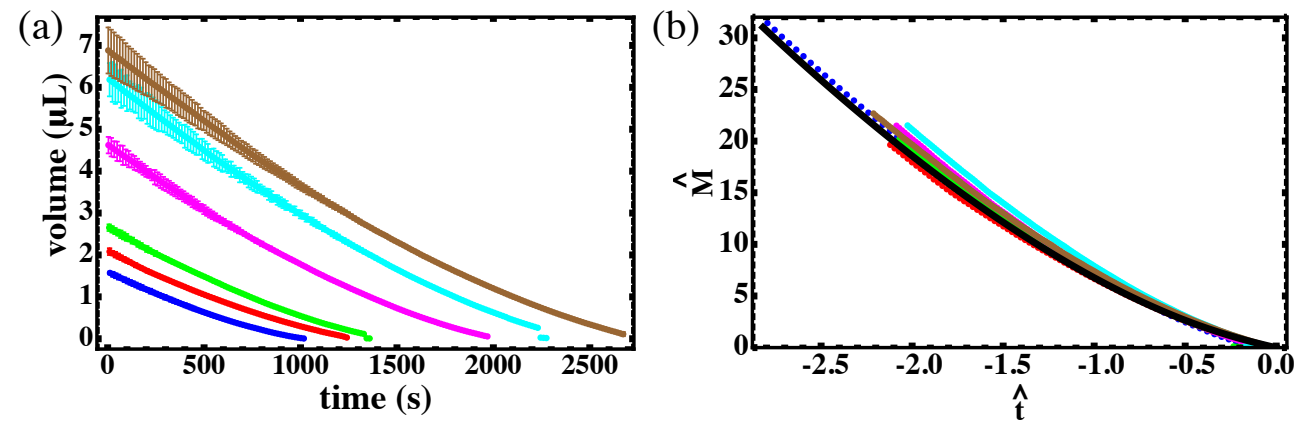

Figure 2.3: (a) Drop volume versus time for initial drop volumes of $1.6 \mu 1$ (blue), $2.1 \mu \mathrm{l}$ (red), $2.9 \mu \mathrm{l}$ (magenta), $4.6 \mu \mathrm{l}$ (green), $6.2 \mu \mathrm{l}$ (cyan), and $6.9 \mu 1$ (brown). The error bars are deduced from the elliptical fit to the data. The measurements were performed with a time resolution of $1 \mathrm{~s}$, but for clarity we show the error bars with a $15 \mathrm{~s}$ resolution. (b) The dimensionless drop mass plotted against the dimensionless time. The black solid line represents the theoretical prediction according to the Popov model. The experimental data is scaled according to (2.6). The time is set to 0 at the end of the drop life (see text).

\subsection{Experimental results}

The drop volume, contact angle, and radius were determined from the experimental data with a time resolution of $1 \mathrm{~s}$. The drop volume versus time plot, clearly shows nonlinear behaviour; see Fig. 2.3(a). Hence, a model based on the small contactangle approximation, which predicts the drop volume to decrease linearly in time $[5,6]$, will not suffice to describe the evolution of the drop volume over time. From the drop volume measurements, the rate of mass loss of the drop $d M / d t$ was derived, as described in Section 2.2. Figure 2.4(a) shows that $d M / d t$ decreases with decreasing contact angle; hence it also decreases in time. Again, nonlinear behaviour is observed, with a steep decline for larger contact angles, but this levels off to a constant rate of mass loss for contact angles smaller than $70^{\circ}$.

During the evaporation, the contact angle of the drops decreases over time from about $150^{\circ}$ to $0^{\circ}$, as shown in Fig. 2.5(a). Initially, the contact angle decreases slowly over time. This is followed by a more rapid, linear decrease over time when the contact angle becomes smaller than approximately $70^{\circ}$. The initial contact angles of the drops differ somewhat owing to irregularities in the substrate. For comparison, not only the experimental data, but also the predictions based on the Popov model are 

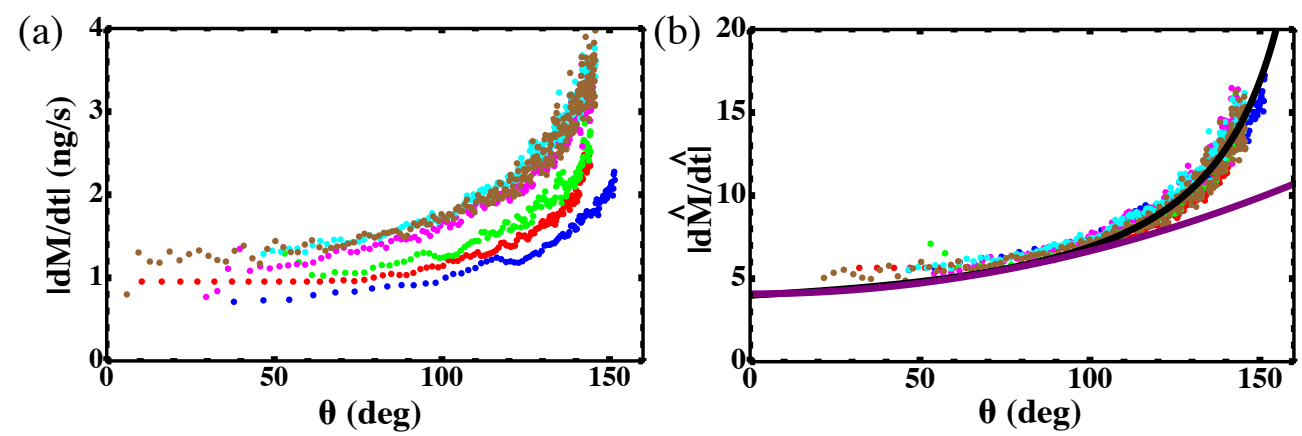

Figure 2.4: (a) The rate of mass loss of the drop (derived from the measured drop volume) versus the contact angle. Colours are as in Fig. 2.3. (b) The same data, but now scaled according to (2.6). Predictions from the Popov model (black solid line) and the model of $\mathrm{Hu} \&$ Larson (purple solid line) are shown.

shown in Fig. 2.5(a). A detailed explanation of this model is given in Section 2.4.

One advantage of the CNF substrates is that the contact lines of the drops remain pinned throughout almost the entire experiment. Therefore, drop evaporation in the constant contact-area mode can be studied, in the absence of any contact-line dynamics. Similar behaviour of the contact angle in the pinned situation has been reported for natural lotus leaves [22], synthetic superhydrophobic surfaces with high contact angle hysteresis [21], and aligned carbon nanotube (CNT) samples [25]. Figure 2.6 shows that depinning only occurs during the final moments of the drop life. In the depinning phase, the contact angle is typically smaller than $40^{\circ}$. Once the drop starts to depin, also the measurements error shoots up, since the contact line does not depin homogeneously, and is therefore no longer exactly circular.

\subsection{Theory of drop evaporation}

To describe theoretically the measured time-evolution of a drop's contact angle and mass, we need to know the evaporative flux from the drop surface. This flux depends on the rate-limiting step in the vapour transport. We assume that vapour transport by free convection, induced by the density difference between dry and humid air [19], is negligible compared to diffusive transport. The influence of evaporative cooling of the drop on the evaporation rate [11] is also neglected. Hence, the vapour transport occurs mainly by diffusive spreading of the water vapour in air, and is characterized by diffusion time $t_{d}=R^{2} / D$, with $R$ the drop radius in the plane of the substrate, 

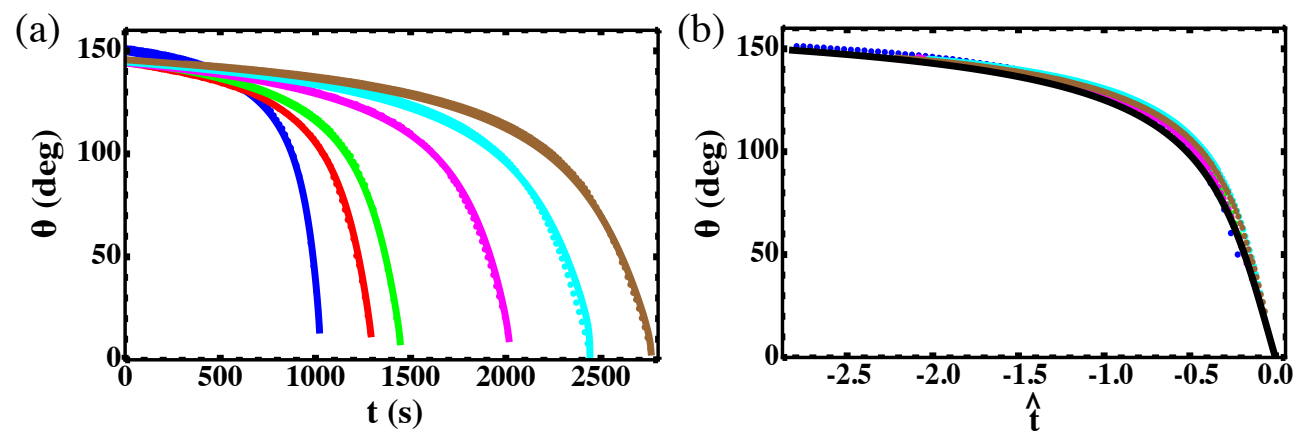

Figure 2.5: (a) The evolution of the contact angle over time. The experimental data $(\bullet)$ can be very well described by the theoretical model of Popov (-), by adjusting the drop radius according to its experimental value (see Section 2.5). The error in the experimental data is not shown, since it is below $1 \%$. (b) The same data, but with the time scaled according to (2.6), and set to 0 at the end of the drop life (see text). The black solid line represents the theoretical prediction according to the Popov model. Colours are as in Fig. 2.3.

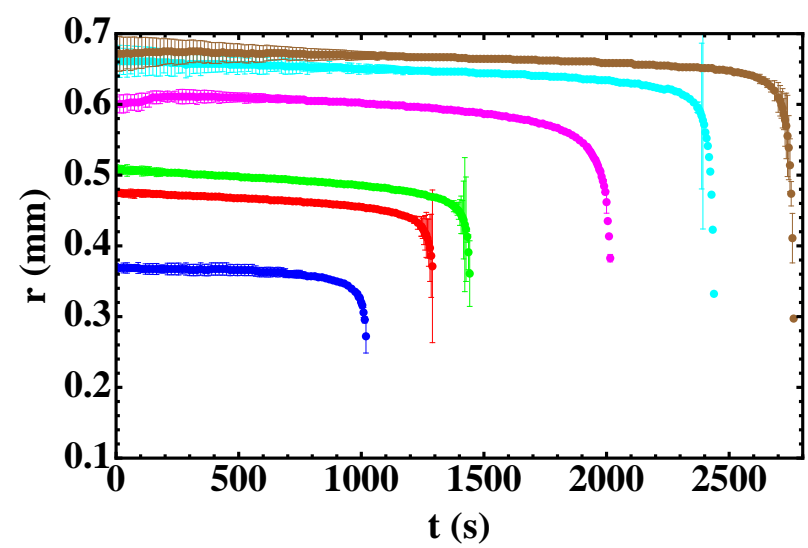

Figure 2.6: The drop radius versus time. Significant depinning of the contact line is observed during the final $4 \%$ of the drop lifetime. Data are shown with $15 \mathrm{~s}$ timeresolution. During the depinning, a resolution of $5 \mathrm{~s}$ is used. Colours are as in Fig. 2.3. 
and $D$ the diffusion coefficient. The diffusion time for water vapour in air is of the order of $10^{-2} \mathrm{~s}$. The evaporation occurs in a quasi-steady fashion: the timescale for diffusion is much smaller than the typical drop evaporation time $t_{f}=\rho_{\ell} /\left(c_{s}-c_{\infty}\right) t_{d}$. Here, $t_{f} / t_{d}=\rho_{\ell} /\left(c_{s}-c_{\infty}\right)$-the density of water divided by the vapour concentration difference between the drop surface and the surroundings- is of the order of $10^{5}$. We do not take into account the Kelvin correction to the vapour pressure, since this effect is negligible for drops of the size considered here.

To determine the diffusive outflux from the drop surface, the vapour concentration field around the drop has to be calculated. We follow the approach taken by Popov [16]. For completeness, we briefly formulate the problem below.

A cylindrical coordinate system $(r, z, \phi)$ is adopted, with $r$ being the radial coordinate, $z$ the direction normal to the substrate, and $\phi$ the circumferential coordinate. The origin of this system is chosen such that $z=0$ corresponds to the substrate, and $r=0$ to the center of the drop. In this case the problem is axisymmetric, i.e. $\phi$ independent. In the quasi-steady, diffusion-limited case the concentration field $c(r, z)$ around the drop is given by

$$
\nabla^{2} c=0
$$

The boundary conditions imposed along the spherical-cap shaped drop with arbitrary contact angle $\theta$ are: (i) $c=c_{s}$, the saturated vapour concentration, along the drop surface; (ii) $c=c_{\infty}$, the ambient vapour concentration, far away from the drop; and (iii) the substrate is impermeable, hence $\partial c / \partial z=0$ along the substrate. The diffusive flux is given by $\boldsymbol{J}=-D \nabla c$. In our experiments, the ambient temperature was $23^{\circ} \mathrm{C}$, the humidity $H=0.3$. At this temperature, $D=24.6 \times 10^{-6} \mathrm{~m}^{2} / \mathrm{s}, \rho_{\ell}=997.6 \mathrm{~kg} / \mathrm{m}^{3}$, and $c_{s}=2.08 \times 10^{-2} \mathrm{~kg} / \mathrm{m}^{3}$ (obtained from [26, p. 6-1, 6-191] by linear interpolation); furthermore $c_{\infty}=H c_{s}$.

In the limit of small contact angles, simplified solutions to (2.1) subject to the boundary conditions (i)-(iii) can be used, as presented by Deegan [5] and Hu \& Larson [6]. In our case a more advanced model is needed, since we consider drops evaporating on a superhydrobic substrate, with initial contact angles of approximately $150^{\circ}$. The analytical solution to the equivalent problem of finding the electric potential around a charged lens-shaped conductor is described in [27]. Popov [16] used this result to determine the rate of mass loss from a drop of arbitrary contact angle:

$$
\frac{d M}{d t}=-\int_{0}^{R} J(r) \sqrt{1+\left(\partial_{r} h\right)^{2}} 2 \pi r d r=-\pi R D\left(c_{s}-c_{\infty}\right) f(\theta),
$$

with $M$ the drop mass, $J$ the diffusive outflux from the drop surface, $h(r, t)$ the drop height, $t$ the time, and

$$
f(\theta)=\frac{\sin \theta}{1+\cos \theta}+4 \int_{0}^{\infty} \frac{1+\cosh 2 \theta \tau}{\sinh 2 \pi \tau} \tanh [(\pi-\theta) \tau] d \tau .
$$


The drop mass can be expressed in terms of $\theta$ by the geometric relation

$$
M(\theta)=\rho_{\ell} \pi R^{3} \frac{\cos ^{3} \theta-3 \cos \theta+2}{3 \sin ^{3} \theta},
$$

which yields an ordinary differential equation for $\theta$ as a function of $t$

$$
\frac{d \theta}{d t}=-\frac{D\left(c_{s}-c_{\infty}\right)}{\rho_{\ell} R^{2}}(1+\cos \theta)^{2} f(\theta) .
$$

Numerical integration then gives $\theta$ as a function of $t$. Once $\theta$ is known, $M(\theta)$ and $d M / d t$ can be derived.

In Fig. 2.3(a)-2.5(a) we showed the evolution of the drop mass and contact angle in time for various drop sizes. Based on the theory described above, one would expect a universal behaviour that is independent of the drop size and the other problem parameters $c_{s}, H, \rho_{\ell}$, and $D$. To demonstrate this, we introduce the nondimensional mass and time as

$$
\hat{M}=\frac{M}{\rho_{\ell} R^{3}} \quad \hat{t}=\frac{c_{s}-c_{\infty}}{\rho_{\ell}} \frac{t}{R^{2} / D} .
$$

By substituting (2.6) into (2.2)-(2.5), we obtain

$$
\begin{aligned}
\frac{d \hat{M}}{d \hat{t}} & =-\pi f(\theta), \\
\hat{M} & =\pi \frac{\cos ^{3} \theta-3 \cos \theta+2}{3 \sin ^{3} \theta}, \\
\frac{d \theta}{d \hat{t}} & =-(1+\cos \theta)^{2} f(\theta) .
\end{aligned}
$$

The relations (2.7)-(2.9) no longer depend on the size of the drops, but only on the contact angle. This implies that when we rescale the experimental data according to (2.6), they should all collapse onto the theoretical curves described by (2.7)-(2.9).

\subsection{Comparison between theory and experiment}

In Section 2.4 we explained that it should be possible to collapse the experimental data for all drop sizes measured onto a single theoretical curve. In order to test this, we have to scale the experimental data according to (2.6). As a characteristic length scale, we would like to use the drop radius. However, during the final moments of the drop's lifetime, the drop radius is a time-dependent quantity. Therefore, we discarded all data where the drop radius was changing significantly $(>10 \%)$ in the results that follow, and used the initial drop radius for scaling. 
The most direct prediction from the Popov model, which involves no time-integration, is the dependence of the rate of mass loss on the contact angle (2.7). Indeed, the scaled experimental data collapse onto a single curve, which is in excellent agreement with the theoretical prediction (2.7), as shown in Fig. 2.4(b). For comparison, the result obtained from applying the model of $\mathrm{Hu}$ and Larson [6] is also shown. Their approximation works well up to $\theta=90^{\circ}$, but for larger contact angles Popov's fully analytical model is required to adequately describe the data.

Figure 2.5(b) shows that the experimental data for the contact angle versus (dimensionless) time follow a universal theoretical curve for all drop sizes measured. The total time it takes a drop to evaporate depends on its initial contact angle, as explained in Section 2.4. Since the initial contact angles vary somewhat, the drop lifetimes differ. However, the experimental time is not an absolute measure, and we therefore have the freedom to set $t=0$ at whichever contact angle we want. As the reference point, we chose $t=0$ at the end of the evaporation process, which is characterized by $\theta=0$. This point is found by linear extrapolation from the last data points measured to $\theta=0$.

Once the contact angle in time is known, we can apply relation (2.8) to derive the drop mass theoretically. Experimentally, the drop mass is obtained independently of the contact angle. Therefore, the comparison between the theoretical predictions and the experimental data for the drop mass, as in Fig. 2.3(b), provides a second validation of the model.

In the results described above, we used the experimental data as long as the contact line remained pinned and hence the drop radius remained constant. In Fig. 2.6, we showed that depinning occurs during the final moments of the drop's lifetime. To construct the theoretical curves in Fig. 2.5(a), this radius change has been taken into account. Time integration was performed backwards in time, starting from the smallest contact angle measured. The agreement between the model results and the experimental data is surprisingly good, even in the regime where the drop radius is changing significantly. Although the drop radius decreases rapidly, the timescale over which the radius shrinks is still large -in the order of $100 \mathrm{~s}$ - compared to diffusion time $\left(10^{-2} \mathrm{~s}\right)$. Therefore, contact-line dynamics is still of negligible influence, and the quasi-steady evaporation model can indeed be applied $[8,18]$.

\subsection{Conclusion}

In this chapter, we studied the evaporation of water drops on superhydrophobic Carbon Nanofibre substrates. These substrates allowed us to measure the evolution of the drop mass and contact angle over time, while the contact line remained pinned 
throughout almost the entire experiment. The initial contact angle was as high as $150^{\circ}$, and since it decreases to $0^{\circ}$ during evaporation, a very large range of contact angles could be studied. Therefore, CNF substrates are a very useful tool to study drop evaporation in the absence of contact-line dynamics. In our theoretical analysis, we deduced universal relations for the time-evolutions of the drop mass and contact angle. This universal scaling behaviour is confirmed by our experimental results. Since the experimental data covered almost the entire range of possible contact angles, we have been able to validate the diffusion-based analytical evaporation model presented by Popov [16]. The agreement of our experimental data with this theoretical model -that does not contain any adjustable parameters- is excellent. Therefore, we conclude that in our experiments the evaporation is quasi-static and diffusiondriven, and thermal effects play no role.

Even during the brief depinning phase, the quasi-steady model predicted the experimental data surprisingly well. Hence, a pinned contact line is not a stringent requirement for the applicability of the quasi-steady evaporation model, provided that the radius change takes place on a longer timescale than the diffusion. By contrast, for drops evaporating on complete wetting substrates, a quasi-static drop profile can no longer be assumed and viscous effects influence the evolution of the contact angle over time $[8,28,29]$. It would be interesting to address intermediate cases, in which there is some contact-line motion, so as to establish the range of applicability of the quasi-steady evaporation model.

\section{References}

[1] R. G. Picknett and R. Bexon, "The evaporation of sessile or pendant drops in still air", J. Colloid Interface Sci. 61, 336-350 (1977).

[2] H. Y. Erbil, G. McHale, and M. I. Newton, "Drop evaporation on solid surfaces: Constant contact angle mode", Langmuir 18, 2636-2641 (2002).

[3] K. S. Birdi, D. T. Vu, and A. Winter, "A study of the evaporation rates of small water drops placed on a solid surface”, J. Phys. Chem. 93, 3702-3703 (1989).

[4] S. M. Rowan, M. I. Newton, and G. McHale, "Evaporation of microdroplets and the wetting of solid-surfaces”, J. Phys. Chem. 99, 13268-13271 (1995).

[5] R. D. Deegan, O. Bakajin, T. F. Dupont, G. Huber, S. R. Nagel, and T. A. Witten, "Contact line deposits in an evaporating drop", Phys. Rev. E 62, 756765 (2000). 
[6] H. Hu and R. G. Larson, "Evaporation of a sessile droplet on a substrate", J. Phys. Chem. B 106, 1334-1344 (2002).

[7] C. Bourges-Monnier and M. Shanahan, "Influence of evaporation on contact angle", Langmuir 11, 2820-2829 (1995).

[8] M. Cachile, O. Benichou, C. Poulard, and A. M. Cazabat, "Evaporating droplets", Langmuir 18, 8070-8078 (2002).

[9] S. David, K. Sefiane, and L. Tadrist, "Experimental investigation of the effect of thermal properties of the substrate in the wetting and evaporation of sessile drops", Colloids Surf. A 298, 108-114 (2007).

[10] G. J. Dunn, S. K. Wilson, B. R. Duffy, S. David, and K. Sefiane, "A mathematical model for the evaporation of a thin sessile liquid droplet: Comparison between experiment and theory", Colloids Surf. A 323, 50-55 (2008).

[11] G. J. Dunn, S. K. Wilson, B. R. Duffy, S. David, and K. Sefiane, "The strong influence of substrate conductivity on droplet evaporation", J. Fluid Mech. 623, 329-351 (2009).

[12] H. Hu and R. G. Larson, "Analysis of the microfluid flow in an evaporating sessile droplet”, Langmuir 21, 3963-3971 (2005).

[13] H. Hu and R. G. Larson, "Analysis of the effects of marangoni stresses on the microflow in an evaporating sessile droplet", Langmuir 21, 3972-3980 (2005).

[14] W. D. Ristenpart, P. G. Kim, C. Domingues, J. Wan, and H. A. Stone, "Influence of substrate conductivity on circulation reversal in evaporating drops", Phys. Rev. Lett. 99, 234502 (2007).

[15] R. D. Deegan, O. Bakajin, T. F. Dupont, G. Huber, S. R. Nagel, and T. A. Witten, "Capillary flow as the cause of ring stains from dried liquid drops", Nature 389, 827-828 (1997).

[16] Y. O. Popov, "Evaporative deposition patterns: Spatial dimensions of the deposit", Phys. Rev. E 71, 036313 (2005).

[17] H. Y. Erbil and R. A. Meric, "Evaporation of sessile drops on polymer surfaces: ellipsoidal cap geometry", J. Phys. Chem. B 101, 6867-6873 (1997).

[18] A. M. Cazabat and G. Guéna, "Evaporation of macroscopic sessile droplets", Soft Matter 6, 2591-2612 (2010). 
[19] N. Shahidzadeh-Bonn, S. Rafai, A. Azouni, and D. Bonn, "Evaporating droplets", J. Fluid Mech. 549, 307-313 (2006).

[20] P. Tsai, S. Pacheco, C. Pirat, L. Lefferts, and D. Lohse, "Drop impact upon micro- and nanostructured superhydrophobic surfaces", Langmuir 25, 1229312298 (2009).

[21] S. A. Kulinich and M. Farzaneh, "Effect of contact angle hysteresis on water droplet evaporation from super-hydrophobic surfaces", Appl. Surf. Sci. 255, 4056-4060 (2009).

[22] X. Zhang, S. Tan, N. Zhao, X. Guo, X. Zhang, Y. Zhang, and J. Xu, "Evaporation of sessile water droplets on superhydrophobic natural lotus and biomimetic polymer surfaces", ChemPhysChem 7, 2067-2070 (2006).

[23] M. Sbragaglia, A. M. Peters, C. Pirat, B. M. Borkent, R. G. H. Lammertink, M. Wessling, and D. Lohse, "Spontaneous breakdown of superhydrophobicity", Phys. Rev. Lett. 99, 156001 (2007).

[24] P. Tsai, R. G. H. Lammertink, M. Wessling, and D. Lohse, "Evaporationtriggered wetting transition for water droplets upon hydrophobic microstructures", Phys. Rev. Lett. 104, 116102 (2010).

[25] K. Gjerde, R. T. Rajendra-Kumar, K. Nordstrom-Andersen, J. KjelstrupHansen, K. B. K. Teo, W. I. Milne, C. Persson, K. Molhave, H.-G. Ruabahn, and P. Boggild, "On the suitability of carbon nanotube forests as non-stick surfaces for nanomanipulation”, Soft Matter 4, 392-399 (2008).

[26] D. R. Lide, CRC Handbook of Chemistry and Physics, 90 edition (CRC Press) (2009).

[27] N. N. Lebedev, Special functions and their applications (Prentice-Hall) (1965).

[28] C. Poulard, G. Guéna, A. M. Cazabat, A. Boudaoud, and M. Ben Amar, "Rescaling the dynamics of evaporating drops", Langmuir 21, 8226-8233 (2005).

[29] J. Eggers and L. M. Pismen, "Nonlocal description of evaporating drops", Phys. Fluids 22, 112101 (2010). 


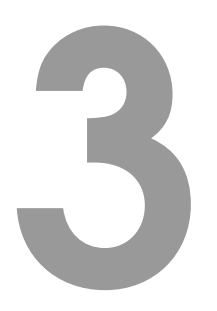

\title{
Stokes flow near the contact line of an evaporating drop *
}

\begin{abstract}
In the previous chapter we have shown that the evaporation of sessile drops in quiescent air is governed by vapour diffusion. For contact angles below $90^{\circ}$, the evaporative flux from the drop tends to diverge in the vicinity of the contact line. Therefore, the description of the flow inside an evaporating drop has remained a challenge. In this chapter, we focus on the asymptotic behaviour near the pinned contact line, by analytically solving the Stokes equations in a wedge geometry of arbitrary contact angle. The flow field is described by similarity solutions, with exponents that match the singular boundary condition due to evaporation. We demonstrate that there are three contributions to the flow in a wedge: the evaporative flux, the downward motion of the liquid-air interface and the eigenmode solution which fulfils the homogeneous boundary conditions. Below a critical contact angle of $133.4^{\circ}$, the evaporative flux solution will dominate, while above this angle the eigenmode solution dominates. We demonstrate that for small contact angles, the velocity field is very accurately described by the lubrication approximation. For larger contact angles, the flow separates into regions where the flow is reversing towards the drop centre.
\end{abstract}

*Published as: Hanneke Gelderblom, Oscar Bloemen, and Jacco H. Snoeijer, "Stokes flow near the contact line of an evaporating drop", J. Fluid Mech. 709, 69-84 (2012). 


\subsection{Introduction}

Evaporation of colloidal dispersion drops is a widely-used mechanism to deposit particles onto a substrate and generate colloidal crystals [1-3]. The ring-shaped stains that remain after evaporation can also be disadvantageous, for example in the coating and inkjet-printing industry [4-7]. To understand and control the stains that form when a drop evaporates, one needs to know the velocity field inside the drying drop [4, 8-10]. The capillary flow inside an evaporating drop is driven by the evaporative mass loss from its surface. There are three mechanisms which can be rate limiting for the evaporation of a drop [11-13]: the transfer of molecules across liquid-air interface, the heat transfer to the interface, or the diffusive transport of the vapour in air. One of the first two mechanisms can be dominant when thin films of evaporating liquid are considered [11,12], when the surrounding phase is not gas but pure vapour $[14,15]$, or for drops on heated substrates $[12,16]$. For macroscopic evaporating drops in air, diffusion-limited evaporation is often assumed, based on estimates of the time scales for transport [12, 17-19]. For water drops there has been some debate about the time scale for transport across the interface, and hence about the applicability of diffusion-limited models [12,13]. Experimentally however, the diffusion-based evaporation model is found to describe the evolution of the drop mass and contact angle of sessile water drops with pinned contact lines very well, for the entire range of possible contact angles [4, 12, 17, 20-22].

Here, we will study the flow near the pinned contact line of a macroscopic evaporating drop in air on an unheated substrate, and therefore we consider diffusionlimited evaporation. Until now, the nature of the flow in the vicinity of the contact line has remained unclear. In the diffusion-limited case, the singular corner geometry of the drop close to the contact line gives rise to a diverging evaporative flux (see Chapter 1), and hence to a diverging velocity field $[4,5,18,23]$. This singularity makes analytical and numerical solutions to the velocity field inside a drop difficult to obtain [15, 23-27]. In several studies the flow inside the drop was solved analytically, but at the expense of smoothing the evaporative flux singularity. Masoud \& Felske [25] considered an exponential cut-off for the flux, while Petsi \& Burganos [26] focussed on uniform evaporation profiles. For small contact angles, evaporation-driven flow inside a drop is often described in the lubrication approximation [19,28], which compares very well with experimental data [8], as will be shown in Chapter 5. It was argued by $\mathrm{Hu} \&$ Larson [23] however, that the standard lubrication approximation does not hold near the contact line region due to the diverging evaporative flux.

On top of that, Marangoni stresses could alter the velocity field in the vicinity of the contact line: the non-uniform evaporative flux leads to temperature gradients over the drop surface, which give rise to differences in surface tension, and drive 
a Marangoni flow inside the drop, as has been confirmed experimentally by $\mathrm{Hu} \&$ Larson [29] for octane drops. Dimensional analysis shows that this Marangoni effect is so strong that it can overcome the diverging evaporation-driven outward flow, at least at some distance from the contact line $[9,29,30]$. However, for water drops the Marangoni effect is found to be weak [29]. In PIV measurements of the velocity field in an evaporating drop by Marín et al. [8] the experimental velocities were of the order of $10 \mu \mathrm{m} / \mathrm{s}$ (see Chapter 5), whereas the Marangoni velocities would be of the order of $10 \mathrm{~mm} / \mathrm{s}$.

In this chapter, we derive analytical solutions of evaporation-driven Stokes flow in a wedge geometry to address the nature of flow near the pinned contact line of an evaporating drop. While solutions to the full flow pattern in the drop can only be obtained numerically $[23,24]$ or for a regularized evaporative flux $[25,26]$, the behaviour in the vicinity of the contact line is characterized by similarity solutions. This is a classical approach for flows near contact lines that goes back to Huh \& Scriven [31] and was recently applied to Marangoni flow in evaporating drops by Ristenpart et al. [9]. We examine the velocity field inside the drop while retaining the singular evaporative flux as a boundary condition. We demonstrate that for small enough contact angle, the lubrication approximation can be applied all the way down to the contact line, which invalidates the argument of $\mathrm{Hu} \&$ Larson [29]. For larger contact angles (above $127^{\circ}$ ) interesting flow structures appear, with a reversal in the flow direction. We show that there are three contributions to the total flow in the wedge: one that comes from the evaporative flux boundary condition, one from the downward movement of the liquid-air interface, and one eigenmode solution, which satisfies the homogeneous boundary conditions. Which of these conditions is dominant depends on the contact angle $\theta$ of the drop. For $\theta<\theta_{c}=133.4^{\circ}$, the critical angle the evaporative flux solution dominates, whereas for $\theta>\theta_{c}$ the eigenmode solution dominates. The solution at the critical point is treated separately. Finally, we comment on the typical pressure in the vicinity of the contact line and on the regularization of the evaporative singularity.

\subsection{Corner solutions}

\subsubsection{Problem formulation}

The geometry of the drop close to the contact line can be approximated by a twodimensional wedge with contact angle $\theta$; see Fig. 3.1. The contact line is located at the origin of the polar coordinate system $(\rho, \phi)$.

We define the velocities in terms of streamfunction $\Psi(\rho, \phi)$ as 
(a)

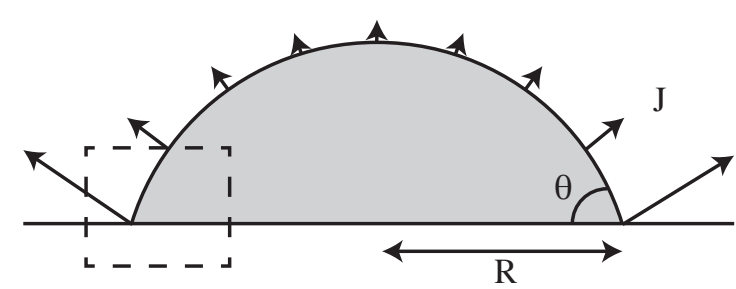

(b)

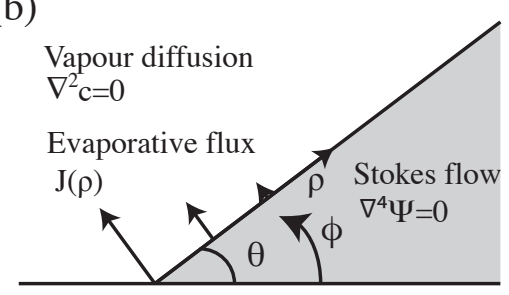

Figure 3.1: (a) An evaporating drop on a substrate with a contact angle $\theta$ and base radius $R$. The arrows indicate the evaporative flux of vapour $J$ from the drop surface to the surroundings. The dashed square marks the area close to the contact line, where the drop geometry can be approximated by a wedge. (b) Overview of the wedge geometry. The evaporative flux drives the Stokes flow inside the liquid. The contact line is located at the origin of the polar coordinate system $(\rho, \phi)$.

$$
u_{\rho}(\rho, \phi)=-\frac{1}{\rho} \frac{\partial \Psi}{\partial \phi}, u_{\phi}(\rho, \phi)=\frac{\partial \Psi}{\partial \rho} .
$$

The flow is governed by the Stokes equations, or equivalently, in terms of the streamfunction, by the biharmonic equation

$$
\nabla^{4} \Psi=0 .
$$

As boundary conditions we have no slip and impermeability of the substrate $(\phi=0)$ on which the drop is deposited

$$
u_{\rho}(\rho, 0)=-\left.\frac{1}{\rho} \frac{\partial \Psi}{\partial \phi}\right|_{\phi=0}=0, \text { and } u_{\phi}(\rho, 0)=\left.\frac{\partial \Psi}{\partial \rho}\right|_{\phi=0}=0,
$$

and no stress at the liquid-air interface $(\phi=\theta)$

$$
\left.\tau_{\rho \phi}\right|_{\phi=\theta}=\eta\left[\rho \frac{\partial}{\partial \rho}\left(\frac{1}{\rho} \frac{\partial \Psi}{\partial \rho}\right)-\frac{1}{\rho^{2}} \frac{\partial^{2} \Psi}{\partial \phi^{2}}\right]=0,
$$

with $\eta$ the dynamic viscosity. The problem is closed by the kinematic boundary condition at the liquid-air interface, where mass transfer due to evaporation occurs. This kinematic boundary condition consists of two contributions. One contribution comes from the evaporative mass flux from the interface, which drives a flow inside the drop. Due to the evaporative mass loss, the drop volume decreases with time. 
While the contact area of the drop remains constant because the contact line is pinned, the contact angle decreases with time. This gives rise to a second contribution to the kinematic boundary condition: the downward motion of the liquid-air interface acts like a closing hinge. Hence, the kinematic boundary condition reads

$$
u_{\phi}(\rho, \theta)=\left.\frac{\partial \Psi}{\partial \rho}\right|_{\phi=\theta}=\frac{1}{\rho_{\ell}} J(\rho)+\frac{d \theta}{d t} \rho,
$$

with $J$ the evaporative flux and $\rho_{\ell}$ the liquid density. Both contributions in (3.5) are known in detail from earlier studies [4, 18]. The key ingredient is that the mass loss from the drop is limited by diffusive transport of the water vapour in the air outside the drop. By solving the vapour concentration field outside the drop, one can find an expression for the evaporative flux $J$, which, close to the contact line, scales as $J \sim \tilde{\rho}^{\lambda(\theta)-1}$ [4], where $\tilde{\rho}=\rho / R$, with $R$ the drop base radius, and

$$
\lambda(\theta)=\frac{\pi}{2 \pi-2 \theta} .
$$

Hence, for $\lambda<1$, which means $\theta<90^{\circ}$, the evaporative flux diverges as the contact line is approached. From the solution for the vapour concentration field in the corner geometry, the evaporative flux is found to be [4]

$$
\frac{J(\rho)}{\rho_{\ell}}=A(\theta) U \tilde{\rho}^{\lambda(\theta)-1}
$$

where $U=D \Delta c / R \rho_{\ell}$ is the velocity scale, which is of order $\mu \mathrm{m} / \mathrm{s}$ for water drops in ambient conditions (see Chapter 5), with $D$ the diffusion constant for vapour in air, and $\Delta c=c_{s}-c_{\infty}$ the vapour concentration difference (in $\mathrm{kg} / \mathrm{m}^{3}$ ) between the drop surface and the surroundings. Prefactor $A(\theta)$ can be found from the asymptotic behaviour of the full spherical-cap solution [18] and is of order unity. The rate of contact angle decrease, $d \theta / d t$, can be determined from the total rate of mass loss from the drop. A closed-form analytical solution for the rate of contact angle decrease was derived by Popov [18] (see Chapter 2)

$$
\frac{d \theta}{d t}=-B(\theta) \frac{U}{R}
$$

with

$$
B(\theta)=(1+\cos \theta)^{2}\left[\frac{\sin \theta}{1+\cos \theta}+4 \int_{0}^{\infty} \frac{1+\cosh 2 \theta \tau}{\sinh 2 \pi \tau} \tanh (\pi-\theta) \tau \mathrm{d} \tau\right] .
$$




\subsubsection{Solution}

For a given power $n$, a general solution to biharmonic equation (3.2) reads [32]

$$
\Psi(\rho, \phi)=\tilde{\rho}^{n}\left[c_{1} \cos n \phi+c_{2} \sin n \phi+c_{3} \cos (n-2) \phi+c_{4} \sin (n-2) \phi\right],
$$

where $n \neq 0,1,2$. Since (3.2) is a linear equation, the two contributions to the inhomogeneous boundary condition (3.5) can be considered separately, whereas the full solution is obtained by superposition. On top of this, one may in principle add the classical "eigenmode" solution of the homogeneous problem by Dean \& Montagnon [33] and Moffatt [34], for which all boundary conditions are zero. Hence, we need to consider three types of solutions: the velocity field due to the evaporative flux condition

$$
u_{\phi}(\rho, \theta)=\frac{1}{\rho_{l}} J(\rho),
$$

the velocity due to the moving interface condition

$$
u_{\phi}(\rho, \theta)=\frac{d \theta}{d t} \rho
$$

and the flow that satisfies the homogeneous condition

$$
u_{\phi}(\rho, \theta)=0 .
$$

From now on, we will refer to (3.11) as the flux condition, to (3.12) as the hinge condition, while the solution satisfying (3.13) is the corner eigenmode. Note that each of these boundary conditions will give rise to a different power of $\tilde{\rho}$ in the final solution. The flux condition (3.11) scales as $\tilde{\rho}^{\lambda-1}$, with $\lambda$ given by (3.6), while the hinge condition (3.12) scales as $\tilde{\rho}$. The homogeneous eigenmode solutions turn out to scale with yet another exponent, denoted $\lambda_{E}$. This exponent also depends on $\theta$, and follows from an eigenvalue equation $M\left(\lambda_{E}, \theta\right)=0$ [34], where

$$
M(\lambda, \theta)=\sin 2(\lambda-1) \theta-(\lambda-1) \sin 2 \theta .
$$

This equation has a countable infinite number of zeros, each of which corresponds to a different eigenmode (with the exception of the trivial solutions $\lambda=0,1,2$ ). The solutions of (3.14) are discussed in great detail by Dean \& Montagnon [33], Moffatt [34] and Moffatt \& Duffy [35]. For angles $\theta<79.6^{\circ}$ (3.14) has complex roots, which causes viscous eddies to appear in the flow [34]. We are interested in the lowest root that has $\operatorname{Re}\left(\lambda_{E}\right)>1$, to ensure regularity of the eigenmode velocity. 


\section{Flux condition}

One can cast the solutions generated by the flux condition (3.11) in the form

$$
\Psi(\rho, \phi)=\frac{R U A(\theta)}{M(\lambda, \theta)} \tilde{\rho}^{\lambda} f(\phi, \theta),
$$

where $\lambda$ depends on $\theta$ as given in (3.6), and the $\phi$-dependent part reads

$$
\begin{aligned}
f(\phi, \theta) & =\frac{1}{2}\{(\lambda-2)[\sin \lambda \theta-\sin (\lambda-2) \theta][\cos \lambda \phi-\cos (\lambda-2) \phi] \\
& \left.+[\lambda \cos \lambda \theta-(\lambda-2) \cos (\lambda-2) \theta]\left[\sin (\lambda-2) \phi-\frac{\lambda-2}{\lambda} \sin \lambda \phi\right]\right\} .
\end{aligned}
$$

The factor $R U$ provides the dimensional strength of the streamfunction, while $A(\theta)$ captures the dependence of the evaporative flux on the contact angle of the drop. Interestingly, the denominator contains the factor $M(\lambda, \theta)$ that was previously defined in (3.14). We thus need to consider separately the cases where $M(\lambda, \theta)=0$, for which the solution (3.15) is not defined.

The function $M(\lambda, \theta)$ has two obvious roots that are encountered in the flux problem, namely $\lambda=1$ and $\lambda=2$. These correspond to cases for which the form (3.10) is degenerate and additional solutions to the biharmonic equation appear. For $\lambda=1$ $\left(\theta=90^{\circ}\right)$ the flux solution becomes

$$
\Psi(\rho, \phi)=\frac{2 R U A(\pi / 2)}{\pi} \tilde{\rho} \phi \sin \phi
$$

while for $\lambda=2\left(\theta=135^{\circ}\right)$ we find

$$
\Psi(\rho, \phi)=\frac{R U A(3 \pi / 4)}{2} \tilde{\rho}^{2}(1-\cos 2 \phi) .
$$

Note that these degenerate solutions are a regular limit of (3.15) for $\theta \rightarrow 90^{\circ}$ and $\theta \rightarrow 135^{\circ}$, respectively.

In addition to these trivial roots, $M(\lambda, \theta)$ with $\lambda=\pi / 2(\pi-\theta)$ exhibits one root that leads to a truly nontrivial solution. This root appears at a critical angle $\theta_{c}=133.4^{\circ}$, with corresponding exponent $\lambda_{c}=1.93$. The critical point arises when the four boundary conditions are not linearly independent, which implies that the inhomogeneous system cannot be solved. Indeed, this is exactly the condition required for a nontrivial (eigenmode) solution of the homogeneous problem, namely $M\left(\lambda_{E}, \theta\right)=0$. As a consequence, the eigenmode solution has the same exponent $\lambda_{E}=\lambda_{c}$ at the critical angle $\theta_{c}$. The resulting critical solution is not of the form (3.10) and will be treated separately in Section 3.2.3. 


\section{Hinge condition}

The hinge condition (3.12) gives rise to a degeneracy of solution (3.10), since in this case $n=2$. The solution to (3.2) with the hinge condition is given by

$$
\Psi(\rho, \phi)=\frac{R U B(\theta)}{N(\theta)} \tilde{\rho}^{2} g(\phi, \theta),
$$

with $N(\theta)=2(2 \theta-\tan 2 \theta)$, and

$$
g(\phi, \theta)=\sin 2 \phi-\tan 2 \theta \cos 2 \phi-2 \phi+\tan 2 \theta .
$$

Hence, (3.19) is defined for all angles, except at $\theta=0^{\circ}$ and $\theta=\theta_{h}=128.7^{\circ}$, where $N(\theta)=0$. For $\theta$ satisfying $N(\theta)=0, \lambda=2$ becomes a double root of eigenvalue equation (3.14). Hence, once more a critical point appears when the eigenmode has the same exponent as the solution of the inhomogeneous hinge problem, i.e. $\lambda_{E}=2$. We anticipate that this critical point will be of less importance than the critical point for the flux condition, as the latter has a slightly smaller exponent $\lambda<2$.

\section{Eigenmode}

The eigenmode represents the nontrivial solution of the homogeneous problem, appearing when $M\left(\lambda_{E}, \theta\right)=0$. It reads [34]

$$
\Psi(\rho, \phi)=R U C(\theta) \tilde{\rho}^{\lambda_{E}} h(\phi, \theta),
$$

with

$$
h(\phi, \theta)=\sin \left[\left(\lambda_{E}-2\right)(\theta-\phi)\right]-\frac{\sin \left(\lambda_{E}-2\right) \theta}{\sin \lambda_{E} \theta} \sin \left[\lambda_{E}(\theta-\phi)\right] .
$$

The prefactor $C(\theta)$ cannot be determined from the "inner" Stokes flow problem, where we consider only the wedge in the vicinity of the contact line. This is fundamentally different from the prefactors $A(\theta), B(\theta)$, which are known from the external boundary conditions on the wedge. In general, the eigenmode solution will be excited by the far-field flow inside the drop, and is therefore determined on the outer scale $R$. This is beyond the present, local analysis. As the only velocity scale in the problem is the one induced by the evaporation, the streamfunction will naturally scale as $R U$, and $C(\theta)$ will be of order unity (with the exception of the critical point). Note that (3.21) is the classical solution by Moffatt [34], which leads to the famous viscous eddies when $\lambda_{E}$ has a nonzero imaginary part. 


\subsubsection{The critical point}

The vanishing denominator of (3.15) at $\theta_{c}=133.4^{\circ}$ and (3.19) at $\theta_{h}=128.7^{\circ}$ signals the breakdown of the local similarity solution. Such a breakdown of similarity solutions in corner flows has been analysed in great detail by Moffatt \& Duffy [35]. This work considered a pressure-driven flow along a duct which cross-section has a sharp corner, as well as a variation to the hinge problem considered above. All cases displayed the same scenario: the inhomogeneous boundary conditions cannot be fulfilled at a critical angle $\theta_{c}$, due to an overlap with the homogeneous eigenmode. The key result by Moffatt \& Duffy [35] is that the critical solution develops logarithmic corrections, of the type $\Psi_{c} \sim \tilde{\rho}^{\lambda_{c}} \ln \tilde{\rho}$. This can be derived by considering the regular solution, including the eigenmode contribution, in the limit $\theta \rightarrow \theta_{c}$. We therefore introduce an expansion parameter, $\varepsilon=\theta-\theta_{c}$, and derive the critical flux solution in the limit $\varepsilon \rightarrow 0$. Below we discuss in detail the critical point for the flux condition, as it will turn out to be the most relevant for the evaporation problem. The critical point for the hinge condition can be treated analogously; the result is given below.

The identity of exponents at the critical point, $\lambda=\lambda_{E}=\lambda_{c}$, forces us to consider a superposition of the flux solution and the eigenmode, $\Psi_{c}=\Psi_{f}+\Psi_{E}$. As expected, the flux solution diverges near the critical point as $1 / \varepsilon$, and gives an expansion of (3.15)

$$
\Psi_{f}=\frac{R U \tilde{\rho}^{\lambda}}{\varepsilon}\left[A_{0}+\varepsilon A_{1}+\mathscr{O}\left(\varepsilon^{2}\right)\right]\left[f_{0}(\phi)+\varepsilon f_{1}(\phi)+\mathscr{O}\left(\varepsilon^{2}\right)\right]
$$

Here we note that $\lambda=\lambda_{c}+\varepsilon \lambda_{1}+\mathscr{O}\left(\varepsilon^{2}\right)$, and all coefficients can in principle be derived from the expressions given in this section. Here we summarize the leadingorder contributions

$$
f_{0}(\phi) \equiv f\left(\phi, \theta_{c}\right), \quad A_{0}=\frac{A\left(\theta_{c}\right)}{M_{1}\left(\lambda_{c}, \theta_{c}\right)},\left.\quad \lambda_{1} \equiv \frac{d \lambda}{d \theta}\right|_{\theta_{c}}=\frac{\pi}{2\left(\pi-\theta_{c}\right)^{2}},
$$

where $f(\phi, \theta)$ was previously defined in (3.16), and $M_{1}\left(\lambda_{c}, \theta_{c}\right)=d M /\left.d \theta\right|_{\theta_{c}}$. As noted by Moffatt \& Duffy [35], a regular solution for $\varepsilon \rightarrow 0$ is only achieved if the eigenmode displays an identical $1 / \varepsilon$ scaling, to compensate for the divergence of $\Psi_{f}$. To leading order, we thus require $C(\theta) \simeq-A_{0} / \varepsilon$, such that the expansion of the eigenmode can be written as

$$
\Psi_{E}=-\frac{R U \tilde{\rho}^{\lambda_{E}}}{\varepsilon}\left[A_{0}+\varepsilon C_{1}+\mathscr{O}\left(\varepsilon^{2}\right)\right]\left[h_{0}(\phi)+\varepsilon h_{1}(\phi)+\mathscr{O}\left(\varepsilon^{2}\right)\right]
$$


where now $\lambda_{E}=\lambda_{c}+\varepsilon \lambda_{E, 1}+\mathscr{O}\left(\varepsilon^{2}\right)$. Once again, we provide the leading-order contributions

$$
h_{0}(\phi) \equiv h\left(\phi, \theta_{c}\right),\left.\quad \lambda_{E, 1} \equiv \frac{d \lambda_{E}}{d \theta}\right|_{\theta_{c}}=-\frac{2\left(\lambda_{c}-1\right)\left[\cos 2\left(\lambda_{c}-1\right) \theta_{c}-\cos 2 \theta_{c}\right]}{2 \theta_{c} \cos 2\left(\lambda_{c}-1\right) \theta_{c}-\sin 2 \theta_{c}}
$$

Indeed, one can verify that $h_{0}(\phi)=f_{0}(\phi)$, which ensures a perfect cancellation of the $1 / \varepsilon$ contributions of $\Psi_{f}$ and $\Psi_{E}$. Finally, the critical solution $\Psi_{c}=\Psi_{f}+\Psi_{E}$ is obtained from (3.23) and (3.25) as

$$
\begin{aligned}
\Psi_{c} & =R U \tilde{\rho}^{\lambda_{c}} \\
& \times\left\{A_{0}\left[\frac{\tilde{\rho}^{\varepsilon \lambda_{1}}-\tilde{\rho}^{\varepsilon \lambda_{E, 1}}}{\varepsilon}\right] f_{0}(\theta)+\left(A_{1}-C_{1}\right) f_{0}(\theta)+A_{0}\left(f_{1}(\phi)-h_{1}(\phi)\right)+\mathscr{O}(\varepsilon)\right\} \\
& =R U A_{0} \tilde{\rho}^{\lambda_{c}}\left\{\left(\lambda_{1}-\lambda_{E, 1}\right) \ln (\tilde{\rho} / \kappa) f_{0}(\phi)+\left[f_{1}(\phi)-h_{1}(\phi)\right]+\mathscr{O}(\varepsilon)\right\}
\end{aligned}
$$

Indeed, this confirms the scenario that the leading-order asymptotics of the critical solution is of the form $\Psi_{c} \sim \tilde{\rho}^{\lambda_{c}} \ln (\tilde{\rho} / \kappa)$. The logarithm appears due to the expansion of $\tilde{\rho}^{\varepsilon \lambda_{1}}-\tilde{\rho}^{\varepsilon \lambda_{E, 1}}$, and thus originates from the "closeness" of $\lambda$ and $\lambda_{E}$ near the critical point. The length scale $\kappa$ that appears inside the logarithm cannot be determined from the current local analysis. Namely, $\kappa$ follows from the combination $\left(A_{1}-C_{1}\right)$ appearing in (3.27). The coefficient $C_{1}$ requires more knowledge of the eigenmode amplitude $C(\theta)$, and thus of the large-scale flow in the sphericalcap shaped drop. Finally, one can verify that this critical solution indeed satisfies the inhomogeneous boundary condition, due to the properties $A_{0} f_{1}=A\left(\theta_{c}\right) / \lambda_{c}$ and $f_{0}=h_{1}=0$ at the free surface $\phi=\theta_{c}$.

The hinge solution at the critical point $\theta_{h}=128.7^{\circ}$ can be obtained following a similar procedure: the singular contributions from the hinge and eigenmode solutions at this point will give rise to logarithmic corrections. Here we only state the result

$$
\Psi_{c, h}=-\frac{R U B\left(\theta_{h}\right)}{8 \theta_{h}^{3}} \tilde{\rho}^{2}\left\{\ln \left(\tilde{\rho} / \kappa_{h}\right) g_{0}(\phi)+\frac{\theta_{h}}{2}\left[g_{1}(\phi)-h_{1}(\phi)\right]+\mathscr{O}(\varepsilon)\right\},
$$

with $g_{0}(\phi) \equiv g\left(\phi, \theta_{h}\right)=\sin 2 \phi-2 \phi+2 \theta_{h}(1-\cos 2 \phi)$. Note that the leading-order part, which contains the logarithmic corrections, is identical to the hinge problem considered by Moffatt \& Duffy [35]. Differences arise in the inhomogeneous contribution, $g_{1}(\phi)$, due to the different boundary conditions. 


\subsection{Results}

\subsubsection{Dominant contribution}

The complete flow field is obtained by a superposition of the flux, hinge and eigenmode solutions identified above. Which of these will be relevant near the contact line depends on the scaling with $\tilde{\rho}$ : the lowest exponent provides the leading-order asymptotic contribution. Figure 3.2 shows the exponents $\lambda$ (flux solution, solid line) and $\lambda_{E}$ (eigenmode, dotted line), as a function of the contact angle $\theta$. For $\theta>\theta_{c}$ the flux solution has the higher exponent, and hence the eigenmode will provide the leading-order contribution. For $\theta<\theta_{c}$, the flux solution dominates. The hinge solution $\sim \tilde{\rho}^{2}$ (dashed line) is asymptotically subdominant for all contact angles. Note that in the vicinity of $\theta_{c}=133.4^{\circ}$, however, the values of all exponents are very close to 2 . This means that in this range of contact angles the asymptotic solution can be reached only at very small $\tilde{\rho}$ : on practical scales all solutions will contribute.

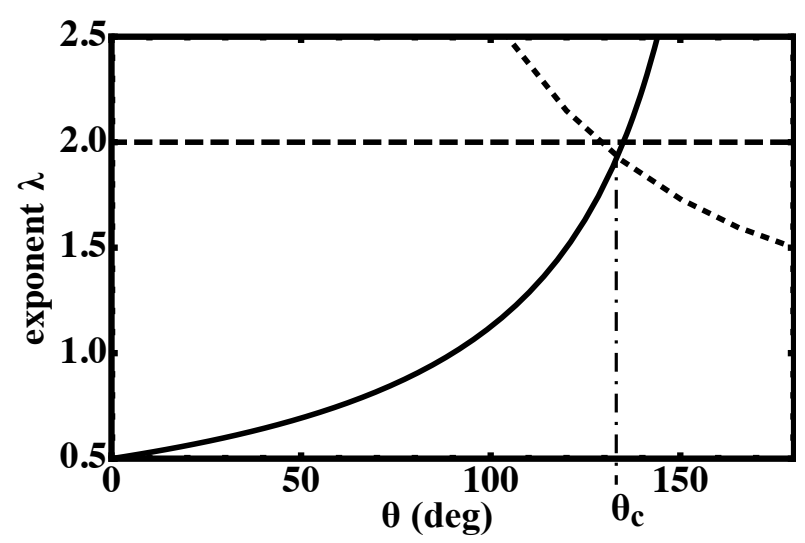

Figure 3.2: A plot of the exponents of $\tilde{\rho}$ that arise in the flux solution (solid line), hinge solution (dashed line) and eigenmode solution (dotted line) versus $\theta$. For $\theta<$ $133.4^{\circ}$, the flux solution is dominant, whereas for larger $\theta$ the eigenmode solution dominates. The hinge solution is always subdominant. The critical points $\theta_{c}=133.4^{\circ}$ and $\theta_{h}=128.7^{\circ}$ arise when respectively the flux or hinge solution is equal to the eigenmode solution.

Most experiments on evaporating drops with pinned contact lines are performed at angles below $90^{\circ}$. This means that the flux solution, for which the prefactor $A(\theta)$ is known, is by far the most relevant case. Nevertheless, an important conclusion is 


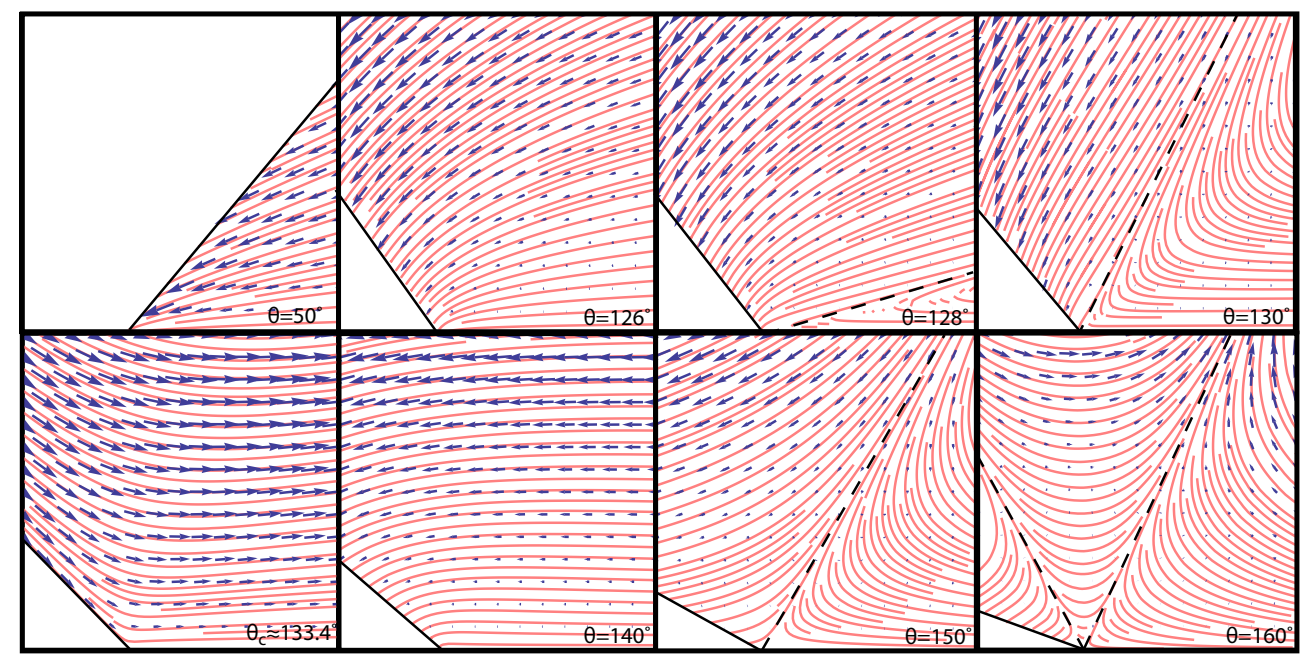

Figure 3.3: Streamline plot of the flux solution (3.15) for different contact angles. For $\theta=128^{\circ}, 130^{\circ}, 150^{\circ}$ and $160^{\circ}$ reversal of the flow direction is observed. Based on (3.30), a new separatrix (dashed line) appears for $\theta \approx 127^{\circ}$ (which disappears at $\left.\theta=\theta_{c}=133.4^{\circ}\right), \theta \approx 148^{\circ}$ and $\theta \approx 157^{\circ}$. At $\theta=\theta_{c}$ we only show the $\phi$-dependent part of the solution, since the prefactor is diverging and has to be treated separately (see Section 3.2.3).

that a local analysis of the problem cannot provide the amplitude of the leading-order flow for $\theta>\theta_{c}$ : the prefactor $C(\theta)$ is determined from matching to the outer flow.

\subsubsection{Streamlines}

The solutions (3.15), (3.19) and (3.21) in principle contain all information on the liquid flow in the vicinity of the contact line. We plot the streamlines associated to these solutions in Figs. 3.3, 3.4 and 3.5 for different values of the contact angle. Fig. 3.3 shows the flow due to the flux condition, with a mass transfer out of the liquid, Fig. 3.4 represents the hinge condition due to the moving liquid-air interface, while Fig. 3.5 shows the eigenmode solutions. For the inhomogeneous solutions, the streamlines arrive at the liquid-air interface with a well-defined angle. As we deal with similarity solutions, the inclination of the streamlines with respect to the free surface is independent of $\rho$ and depends only on $\theta$. The only exception is the solution at the critical point $\theta_{c}$ (3.27), where an additional length scale $\kappa$ from the outer problem comes in and the self-similarity is lost. 


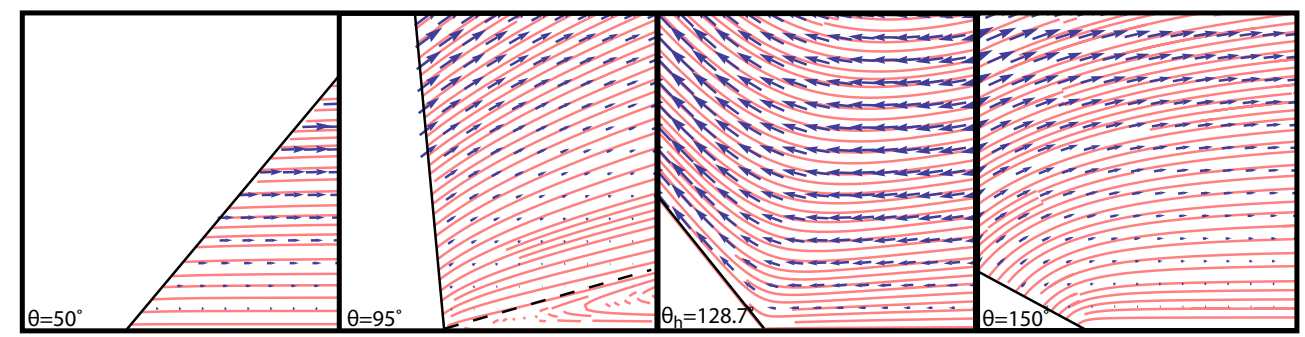

Figure 3.4: Streamline plot of the hinge solution (3.19) for different contact angles. A separatrix (dashed line) appears for $\theta=90^{\circ}$, and disappears again for $\theta=\theta_{h} \approx$ $128.7^{\circ}$. At $\theta=\theta_{h}$ we only show the $\phi$-dependent part of the solution, since the prefactor is diverging and has to be treated separately (see Section 3.2.3).

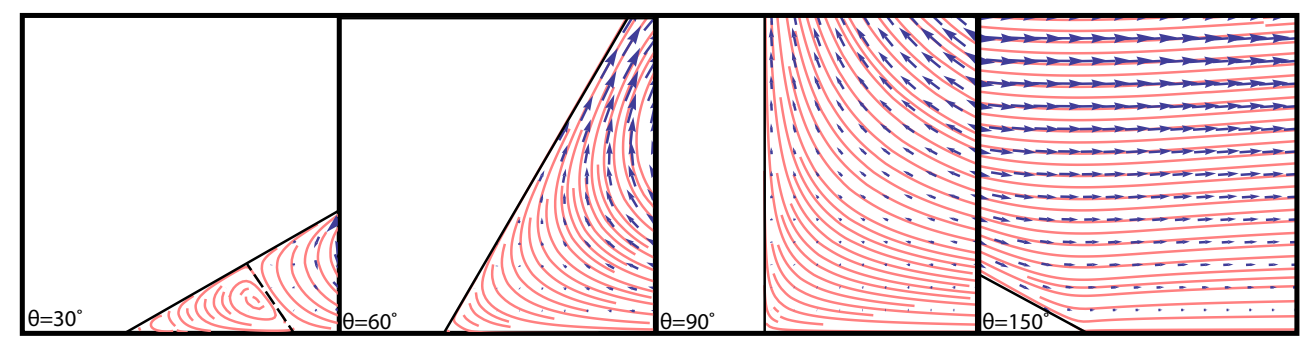

Figure 3.5: Streamline plot of the eigenmode solution (3.21) for different contact angles with prefactor $C(\theta)=1$. For $\theta=30^{\circ}$ and $60^{\circ}$ viscous eddies are present. The dashed line separates the two eddies visible for $\theta=30^{\circ}$.

When analysing the flux solution in Fig. 3.3 in more detail, one can see that for $\theta<90^{\circ}$ the strength of the flow increases upon approaching the contact line. From the scaling in (3.15) one in fact sees that the velocity diverges as $\rho \rightarrow 0$ for this range of contact angles, since $\lambda-1<0$. Above $\theta>90^{\circ}$, however, the velocity decays and vanishes near the contact line. Interestingly, the flow displays some reversal structure for these large contact angles. As $\theta$ increases from $126^{\circ}$ to $128^{\circ}$ the flow becomes separated into two regions, such that near the bottom wall the liquid flow direction is actually away from the contact line, towards the center of the drop. The dashed line shows the separating flow line that ends with a stagnation point at the contact line. The separatrix then moves upwards with increasing $\theta$, as seen in the plot for $\theta=130^{\circ}$. Between $\theta=130^{\circ}$ and $140^{\circ}$ the separatrix disappears. This occurs when the separatrix reaches the free surface, which coincides with the critical 


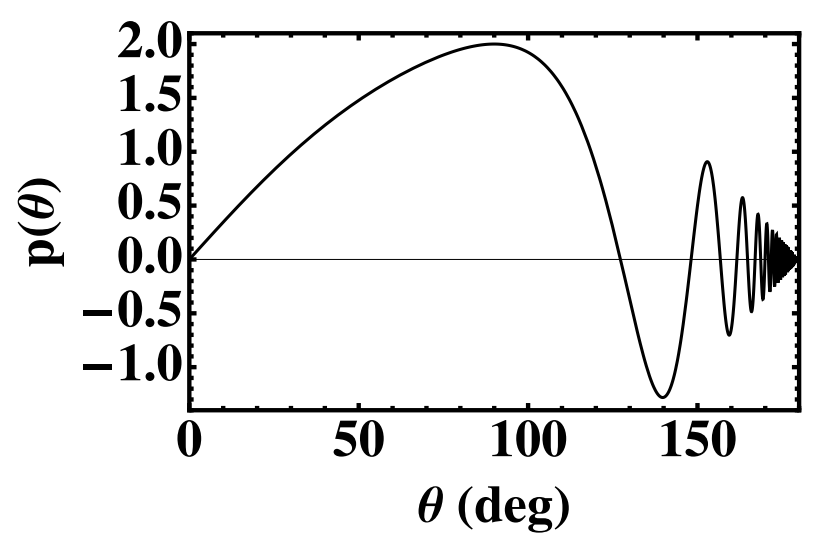

Figure 3.6: The contact angles at which a new separatrix appears for the flux condition are located at the zeros of $p(\theta)=\sin \lambda \theta-\sin (\lambda-2) \theta$, given by (3.30).

point, i.e. $\theta=\theta_{c}=133.4^{\circ}$. At this critical angle, the surface flow changes direction. Indeed, a separatrix located at the free surface is incompatible with the boundary condition of an outward flux, which illustrates the breakdown of a local similarity solution and the appearance of the critical solution (3.27). When the contact angle is increased to $150^{\circ}$ a new separatrix appears. For $\theta=160^{\circ}$ we see that this separatrix has moved upwards, and a second separatrix has appeared. Separatrices that appear for $\theta>133.4^{\circ}$ do not disappear again, since the flux solution (3.15) has only one critical point. One can demonstrate from the exact solutions that a new separatrix appears at $\phi=0$ when $\partial u_{\rho} /\left.\partial \phi\right|_{\phi=0}=0$. This leads to the condition

$$
p(\theta)=\sin \theta(\lambda-2)-\sin \theta \lambda=0,
$$

with $\lambda=\pi / 2(\pi-\theta)$, which is illustrated in Fig. 3.6. From criterion (3.29) the contact angles at which a new separatrix appears are found to be (in radians)

$$
\theta=\frac{\pi}{4}\left(\sqrt{1+6 k+k^{2}}+1-k\right), \text { for } k=1,3,5 \ldots
$$

The hinge solutions have a simpler structure (Fig. 3.4). For all values of $\theta$ we find that $u \sim \tilde{\rho}$, and thus the speed increases linearly with the distance from the contact line. For contact angles below $90^{\circ}$ the flow is oriented away from the contact line. For $\theta>90^{\circ}$ only one single separatrix appears, which has disappeared again for $\theta>128.7^{\circ}$. At the critical point $\theta_{h}=128.7^{\circ}$ the local similarity solution again breaks down, and we encounter a critical solution. 
The eigenmode solutions are shown in Fig. 3.5, where we took $C(\theta)=1$. In the eigenmode solution, viscous eddies will appear for angles smaller than $79.6^{\circ}$ [34]; in that case the real part, $\operatorname{Re}\left(\lambda_{E}\right)>3.8$, so that the eigenmode is subdominant with respect to the inhomogeneous solutions. Hence, in the evaporation problem, the eigenmodes become important only for angles where no eddies are present.

\subsubsection{Lubrication limit: $\theta \ll 1$}

Now we have the full analytical solution to the evaporation-driven velocity field in a wedge available, we can check whether for sufficiently flat drops (i.e. small contact angles) the lubrication approximation can be applied. Therefore, we expand the Stokes flow solution for the flux condition for small $\theta, \phi$, yielding

$$
u_{\rho}(\rho, \phi) \simeq 3 A(\theta) U \frac{1}{\theta} \frac{1}{(\rho / R)^{1-\lambda(\theta)}}\left[\frac{1}{2}\left(\frac{\phi}{\theta}\right)^{2}-\frac{\phi}{\theta}\right] .
$$

In the lubrication approximation, we can write the radial velocity in terms of the radial distance from the drop center, $r$, distance to the solid substrate, $z$, and drop height $h$, as $[8,23]$ (see Chapter 5)

$$
u_{r}(r, z)=-\frac{3 \sqrt{2}}{\pi} U \frac{1}{\theta} \frac{1}{\sqrt{1-(r / R)}}\left[\frac{1}{2}\left(\frac{z}{h}\right)^{2}-\frac{z}{h}\right] .
$$

For small enough $\theta, A(\theta) \rightarrow \sqrt{2} / \pi[18]$ and $\tilde{\rho} \simeq 1-r / R$. Furthermore, since $z=\rho \sin \phi \simeq \rho \phi$ and $h=\rho \sin \theta \simeq \rho \theta, z / h \simeq \phi / \theta$, and (3.31) leads to the same expression as (3.32). Hence, the Stokes solution converges to the lubrication solution for small $\theta$. To illustrate this, we plotted the lubrication solution together with the Stokes solution for different contact angles; see Fig. 3.7. For convenience, we normalized all velocities by the velocity at the interface. From these results it is clear that even close to the contact line, where the evaporative flux is diverging, the lubrication approximation can be applied.

\subsection{Discussion}

We have derived the analytical solution to the Stokes-flow problem in a wedge geometry with an evaporative flux boundary condition. From the Stokes-flow solution it was found that the lubrication approximation accurately describes the velocity field in an evaporating drop all the way down to the contact line, for small enough contact angle. For larger contact angles, reversing flow structures are observed, and the 


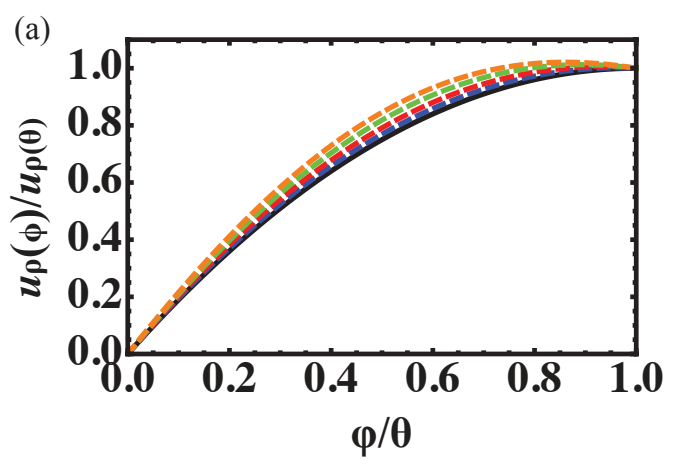

(b)

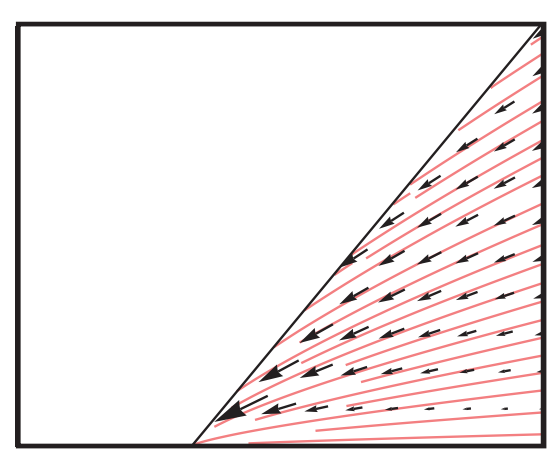

Figure 3.7: (a) The radial velocity $u_{\rho}(\phi)$, normalized by the velocity at the free surface $u_{\rho}(\theta)$, in the lubrication approximation (3.32) (solid black line), and the Stokes flow solutions (3.1) (dashed lines) for $\theta=20^{\circ}$ (blue), $\theta=30^{\circ}$ (red), $\theta=40^{\circ}$ (green), $\theta=50^{\circ}$ (orange). (b) Streamline plot of the lubrication solution for $\theta=50^{\circ}$. The velocity in azimuthal direction is derived from the continuity equation.

eigenmode solution of the problem becomes of importance. Hence, the direction of the flow (towards or away from the contact line) depends on the contact angle of the drop. The contact angles at which new separatrices in the velocity field appear are calculated from the exact solutions. This flow reversal, the appearance of separatrices and the dominance of the eigenmode solution for evaporation-driven flow have not previously been reported in numerical studies [23, 24], which focussed on smaller angles, nor in analytical studies $[25,26]$, where different boundary conditions were used. It would be interesting to investigate these aspects further in numerical simulations of the flow in the entire drop, in particular near the critical point, where the exponents of all three contributions (flux, hinge and eigenmode) to the total solution are very close.

The direction of the surface flow in the evaporating drops changes when the contact angle passes through the critical angle $\theta_{c}$. In addition, Marangoni stresses induced by the non-uniform evaporative flux can change the surface flow direction. We can re-examine the influence of the Marangoni effect by direct comparison of our results to the solution with a Marangoni boundary condition, presented by Ristenpart et al. [9]. In our case, hence in absence of Marangoni stresses, the radial velocity scales as

$$
u_{\rho} \sim \frac{1}{\theta} U \tilde{\rho}^{\lambda-1}
$$


whereas the solution for the Marangoni case scales as [9]

$$
u_{\rho} \sim \theta^{2} U \operatorname{Ma} \tilde{\rho}^{\lambda}
$$

where $\mathrm{Ma}=\rho_{\ell} \beta R \Delta H_{v} / \mu k$ the Marangoni number, with $\Delta H_{v}$ the specific latent heat of evaporation, $\beta$ the dependence of surface tension on the temperature, and $k$ the thermal conductivity of the liquid. For water, the Marangoni number is of order $10^{5}$. The factors $1 / \theta$ and $\theta^{2}$ only appear in the small contact-angle limit, otherwise, the $\theta$-dependent prefactors are of order unity. From (3.33) and (3.34) it is clear that the two expressions have a different power in $\rho$, and therefore a cross-over length scale exists. Below this critical length $\rho_{c}$, the lowest power, hence the evaporationdriven solution (3.33), will dominate, above this length the Marangoni-driven flow is dominant. The cross-over length scale is given by

$$
\rho_{c}=\frac{1}{\theta^{3} \mathrm{Ma}} R
$$

hence, this length scale is of order $10^{-8} \mathrm{~m}$ for a millimetre-sized drop, as long as the contact angle is of order 1. Only for extremely small contact angles, the length scale over which evaporation dominates the Marangoni effect becomes comparable to the drop size. Hence, the Marangoni effect should alter the velocity pattern in evaporating drops significantly, as described by the solutions obtained by Ristenpart et al. [9]. The question why this is not observed experimentally for water drops remains, with as possible explanation that it has to do with surface-active contamination which could suppress the Marangoni effect [29].

We described flow structures inside drops on partially wetting substrates, in cases where the contact line is pinned. When the contact line is not pinned but free to move, this will lead to an additional velocity field inside the drop, as described by Huh \& Scriven [31]. Since the Stokes flow problem is linear, the moving contactline solution by Huh \& Scriven [31] could just be superimposed on our evaporationdriven solution. In that case, two velocity fields that are in opposite directions will arise in the problem: one with a mean flow directed towards the contact line (for contact angles below $127^{\circ}$ ), driven by the evaporative flux from the drop surface, and one with a mean flow away from the contact line driven by the receding contact line. Since the evaporation-driven velocity field diverges close to the contact line for $\theta<90^{\circ}$, it will dominate the solution close to the contact line. A cross-over length can be defined where both velocity fields cancel each other [28]. This length depends on the contact angle and can be as large as $100 \mu \mathrm{m}$ : for small contact angles the entire flow field in the wedge close to the contact line will be dominated by evaporation.

Interestingly, the diverging evaporative flux leads not only to a diverging velocity field inside the drop, but also to a diverging pressure field, which scales as $p \sim$ 
$1 / \theta(\eta U / R) \tilde{\rho}^{\lambda-2}$. For water drops, with $U=10^{-6} \mathrm{~m} / \mathrm{s}, \eta=10^{-3} \mathrm{~Pa} \mathrm{~s}, R=10^{-3} \mathrm{~m}$, this gives for very small length scales $\tilde{\rho}=10^{-6}\left(\rho=10^{-9} \mathrm{~m}\right)$ that $p=10^{3} / \theta \mathrm{Pa}$. This pressure is large compared to the Laplace pressure $p_{\gamma}=\gamma / R=10^{2} \mathrm{~Pa}$. Therefore, we expect on small scales $(\rho \sim \mathrm{nm})$ some additional curvature of the interface. This subtle problem has been studied in detail by Berteloot et al. [28]: for angles $\sim 0.1$ a change in contact angle of about $15 \%$ was reported. For angles of order unity, however, no significant effect of the evaporation-driven flow on the contact angle was found. Note that, since $p \sim \tilde{\rho}^{\lambda-2}$, the pressure will diverge for all contact angles, since the leading-order exponent $\lambda<2$ (see Fig. 3.2). For $\theta<90^{\circ}$, or $\lambda<1$, the evaporation problem is even more singular than the moving contact-line problem by Huh \& Scriven [31], for which $p \sim \tilde{\rho}^{-1}$. Moreover, the introduction of a slip length does not change the pressure exponent given by the evaporative-flux boundary condition, contrary to the case of a moving contact line of a non-volatile liquid.

Finally, it is still an open question what regularizes the radially diverging velocity inside an evaporating drop with a contact angle smaller than $90^{\circ}$. In previous studies, two approaches have been used. Either the evaporative flux singularity is smoothed mathematically [24-27], or by the introduction of a precursor film, which regularizes the singular wedge geometry near the contact line [19, 27, 36, 37]. However, for drops on a hydrophobic substrate, a precursor film is not expected, and a proper explanation for the regularization of the evaporative flux is still lacking. The large pressure close to the contact line could reduce the vapour concentration, according to the Kelvin equation [19] but for contact angles of order unity the pressure appears insufficient for this effect, even at the nanometre scale. Furthermore, if the rate at which molecules are transported by diffusion exceeds the rate at which they transfer across the interface, the air just above the drop is no longer saturated with vapour and the evaporative flux no longer diverges. We speculate that another option could be that the regularization occurs on the length scale of the mean free path of the water vapour molecules in the surrounding air, which is of the order of $100 \mathrm{~nm}$, and sets a lower bound on the validity of the diffusion problem.

\section{References}

[1] T. P. Bigioni, X. M. Lin, T. T. Nguyen, E. I. Corwin, T. A. Witten, and H. M. Jaeger, "Kinetically driven self assembly of highly ordered nanoparticle monolayers", Nat. Mater. 5, 265-270 (2006).

[2] E. Dufresne, E. Corwin, N. A. Greenblatt, J. Ashmore, D. Wang, A. Dinsmore, J. Cheng, X. Xie, J. Hutchinson, and D. Weitz, "Flow and fracture in drying nanoparticle suspensions", Phys. Rev. Lett. 91, 224501 (2003). 
[3] K. P. Velikov, C. G. Christova, R. P. A. Dullens, and A. van Blaaderen, "Layerby-layer growth of binary colloidal crystals", Science 296, 106 -109 (2002).

[4] R. D. Deegan, O. Bakajin, T. F. Dupont, G. Huber, S. R. Nagel, and T. A. Witten, "Capillary flow as the cause of ring stains from dried liquid drops", Nature 389, 827-828 (1997).

[5] R. D. Deegan, O. Bakajin, T. F. Dupont, G. Huber, S. R. Nagel, and T. A. Witten, "Contact line deposits in an evaporating drop", Phys. Rev. E 62, 756765 (2000).

[6] H. B. Eral, D. M. Augustine, M. H. G. Duits, and F. Mugele, "Suppressing the coffee stain effect: how to control colloidal self-assembly in evaporating drops using electrowetting", Soft Matter 7, 1-5 (2011).

[7] P. J. Yunker, T. Still, M. A. Lohr, and A. G. Yodh, "Suppression of the coffeering effect by shape-dependent capillary interactions", Nature 476, 308-311 (2011).

[8] A. G. Marín, H. Gelderblom, D. Lohse, and J. H. Snoeijer, "Order-to-disorder transition in ring-shaped colloidal stains”, Phys. Rev. Lett. 107, 085502 (2011).

[9] W. D. Ristenpart, P. G. Kim, C. Domingues, J. Wan, and H. A. Stone, "Influence of substrate conductivity on circulation reversal in evaporating drops", Phys. Rev. Lett. 99, 234502 (2007).

[10] D. Brutin, B. Sobac, B. Loquet, and J. Sampol, "Pattern formation in drying drops of blood", J. Fluid Mech. 667, 85-95 (2011).

[11] B. Haut and P. Colinet, "Surface-tension-driven instabilities of a pure liquid layer evaporating into an inert gas", J. Colloid Interface Sci. 285, 296-305 (2005).

[12] A. M. Cazabat and G. Guéna, "Evaporation of macroscopic sessile droplets", Soft Matter 6, 2591-2612 (2010).

[13] N. Murisic and L. Kondic, "On evaporation of sessile drops with moving contact lines”, J. Fluid Mech. 679, 219-246 (2011).

[14] J. P. Burelbach, S. G. Bankoff, and S. H. Davis, "Nonlinear stability of evaporating condensing liquid films", J. Fluid Mech. 195, 463-494 (1988). 
[15] P. Colinet and A. Rednikov, "On integrable singularities and apparent contact angles within a classical paradigm", Eur. Phys. J. Spec. Top. 197, 89-113 (2011).

[16] D. Anderson and S. Davis, "The spreading of volatile liquid droplets on heated surfaces”, Phys. Fluids 7, 248-265 (1995).

[17] H. Hu and R. G. Larson, "Evaporation of a sessile droplet on a substrate", J. Phys. Chem. B 106, 1334-1344 (2002).

[18] Y. O. Popov, "Evaporative deposition patterns: Spatial dimensions of the deposit”, Phys. Rev. E 71, 036313 (2005).

[19] J. Eggers and L. M. Pismen, "Nonlocal description of evaporating drops", Phys. Fluids 22, 112101 (2010).

[20] G. Guéna, C. Poulard, and A. M. Cazabat, "The leading edge of evaporating droplets", J. Colloid Interface Sci. 312, 164-171 (2007).

[21] H. Gelderblom, A. G. Marín, H. Nair, A. van Housselt, L. Lefferts, J. H. Snoeijer, and D. Lohse, "How water droplets evaporate on a superhydrophic substrate”, Phys. Rev. E 83, 026306 (2011).

[22] B. Sobac and D. Brutin, "Triple-line behavior and wettability controlled by nanocoated substrates: influence on sessile drop evaporation", Langmuir 27, 14999-15007 (2011).

[23] H. Hu and R. G. Larson, "Analysis of the microfluid flow in an evaporating sessile droplet”, Langmuir 21, 3963-3971 (2005).

[24] B. J. Fischer, "Particle convection in an evaporating colloidal droplet", Langmuir 18, 60-67 (2002).

[25] H. Masoud and J. D. Felske, "Analytical solution for stokes flow inside an evaporating drop: spherical and cylindrical cap shapes", Phys. Fluids 21, 0421021-042102-1 (2009).

[26] A. J. Petsi and V. N. Burganos, "Stokes flow inside an evaporating liquid line for any contact angle", Phys. Rev. E 78, 036324-1-036324-9 (2008).

[27] C. Poulard, G. Guéna, A. M. Cazabat, A. Boudaoud, and M. Ben Amar, "Rescaling the dynamics of evaporating drops", Langmuir 21, 8226-8233 (2005). 
[28] G. Berteloot, C. T. Pham, A. Daerr, F. Lequeux, and L. Limat, "Evaporationinduced flow near a contact line: consequences on coating and contact angle", Europhys. Lett. 83, 14003 (2008).

[29] H. Hu and R. Larson, "Marangoni effect reverses coffee-ring depositions", J. Phys. Chem. B 110, 7090-7094 (2006).

[30] H. Bodiguel and J. Leng, "Imaging the drying of a colloidal suspension", Soft Matter 6, 5451-5460 (2010).

[31] C. Huh and L. E. Scriven, "Hydrodynamic model of steady movement of a solid/liquid/fluid contact line", J. Colloid Interface Sci. 35, 85-101 (1971).

[32] J. H. Michell, "On the direct determination of stress in an elastic solid, with application to the theory of plates", Proc. London Math. Soc. 100, 100-124 (1899).

[33] W. R. Dean and P. E. Montagnon, "On the steady motion of viscous liquid in a corner”, Proc. Camb. Phil. Soc. 45, 389-394 (1949).

[34] H. K. Moffatt, "Viscous and resistive eddies near a sharp corner", J. Fluid Mech. 18, 1-18 (1964).

[35] H. K. Moffatt and B. R. Duffy, "Local similarity solutions and their limitations", J. Fluid Mech. 96, 299-313 (1980).

[36] C. T. Pham, G. Berteloot, F. Lequeux, and L. Limat, "Dynamics of complete wetting liquid under evaporation", Europhys. Lett. 92, 54005 (2010).

[37] S. Semenov, V. M. Starov, M. G. Velarde, and R. G. Rubio, "Droplets evaporation: Problems and solutions”, Eur. Phys. J. Spec. Top. 197, 265-278 (2011). 


\section{4}

\section{Stokes flow in a drop evaporating from a liquid subphase *}

In the previous chapter, we have studied the nature of the flow near the contact line of a drop evaporating from a flat solid substrate. In this chapter, the evaporation of a drop from a liquid subphase is investigated. The two liquids are immiscible, and the contact angles between them are given by the Neumann construction. The evaporation of the drop gives rise to flows in both liquids, which are coupled by the continuity of velocity and shear-stress conditions. We derive self-similar solutions to the velocity fields in both liquids close to the three-phase contact line, where the drop geometry can be approximated by a wedge. We focus on the case where Marangoni stresses are negligible, for which the flow field consists of three contributions: flow driven by the evaporative flux from the drop surface, flow induced by the receding motion of the contact line, and an eigenmode flow that satisfies the homogeneous boundary conditions. The eigenmode flow is asymptotically subdominant for all contact angles. The moving contact-line flow dominates when the angle between the liquid drop and the horizontal surface of the liquid subphase is smaller than $90^{\circ}$, while the evaporativeflux driven flow dominates for larger angles. A parametric study is performed to show how the velocity fields in the two liquids depend on the contact angles between the liquids and their viscosity ratio.

\footnotetext{
${ }^{*}$ Submitted to Phys. Fluids as: Hanneke Gelderblom, Howard A. Stone, and Jacco H. Snoeijer, "Stokes flow in a drop evaporating from a liquid subphase".
} 


\subsection{Introduction}

The evaporation of a liquid drop from a flat solid substrate has been studied extensively, for example in the context of evaporation-driven particle deposition [1-4] and evaporative cooling [5-7]. However, apparently little is known about the evaporation of liquid drops from a liquid subphase. On a liquid subphase the contact line of the drop is not pinned, the subphase is deformable, and the evaporation can generate a flow in both liquid phases. Examples of such a system are gasoline or oil drops on water, or water drops on mercury. Similar interface deformations occur for drops evaporating from soft, deformable solid substrates [8-11], for which the flow geometry is reminiscent of drops floating on another liquid subphase.

Evaporation of sufficiently small sessile drops under atmospheric conditions is usually governed by the diffusive transport of vapour in the surrounding air [1, 1217]. The evaporative flux from the drop surface drives a radially outward flow inside the drop, which is responsible for the so-called coffee-stain effect [1,2]. In drops where the contact line is free to move, there is an additional contribution to the flow field that comes from the receding motion of the contact line [14, 18]. Furthermore, the non-uniform evaporation of sessile drops leads to temperature gradients on the liquid-air interface. The subsequent Marangoni flows can give rise to additional circulation $[5,19,20]$.

It has remained a challenge to characterize the flow near the contact line, due to the small length scales involved and the evaporative singularity. In Chapter 3, we have studied the nature of the flow near the pinned contact line of a liquid drop evaporating from a flat solid surface by approximating the drop profile by a wedge geometry [21]. We have shown that the Stokes flow in this wedge-shaped region can be described by similarity solutions and consists of three contributions: flow driven by the evaporative flux from the drop surface, flow induced by the downward motion of the liquid-air interface, and an eigenmode flow that satisfies the homogeneous boundary conditions and can give rise to Moffatt corner eddies [22].

In this chapter, we investigate the evaporation of a liquid drop from a liquid subphase, as shown in Fig. 4.1(a). We consider drops with a size smaller than the capillary length. In that case, the interface of the liquid subphase remains horizontal and the drop attains the shape of a lens. The angles $\theta, \alpha$, and $\beta$ between the liquids are dictated by the three surface tensions in the system via the Neumann construction, such that both the horizontal and vertical components of the capillary forces add to zero [23]. We focus again on the nature of the flow near the contact line of the drop, where the drop shape can be approximated by a wedge geometry, see Fig. 4.1(b). The evaporation of liquid 1 will cause a flow in both liquids 1 and 2. We describe these flows by deriving self-similar solutions to the Stokes equations in a wedge geometry, 
(a)

gas

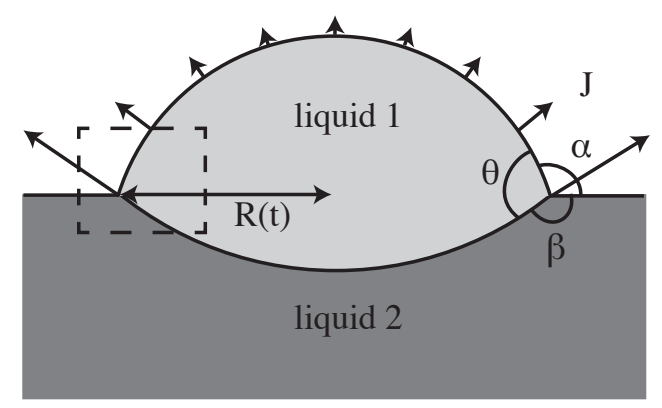

(b)

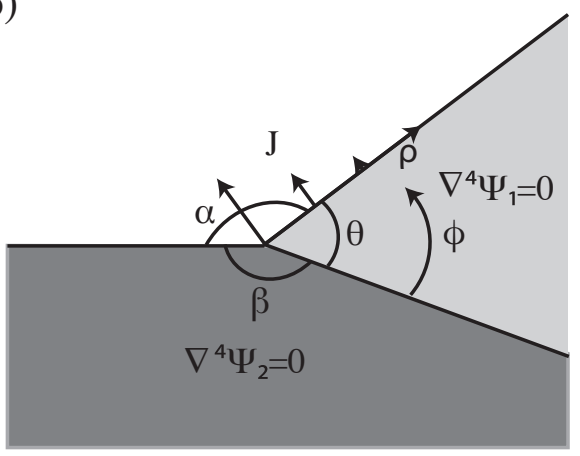

Figure 4.1: (a) A drop of liquid 1 evaporating from a non-volatile liquid subphase (liquid 2). The angles $\theta, \alpha$, and $\beta$ between the two liquids can be found from the Neumann construction at the three-phase contact line. The dashed square marks the area close to the contact line, where the drop shape can be approximated by a wedge. (b) An overview of the wedge geometry containing both liquid 1 and liquid 2. The contact line is located at the origin of the polar coordinate system $(\rho, \phi)$. The evaporative flux $J$ (indicated by the arrows) from the surface of liquid 1 drives the flow inside liquid 1 , which is coupled to the flow in liquid 2 via the continuity of velocity and shear stress conditions.

in which the flows in both phases are coupled dynamically. Again, the flow in the wedge consists of three contributions: the evaporative-flux driven flow, the flow due to the receding motion of the contact line, and the eigenmode flow for the coupled phases. The eigenmode flow in a similar double-wedge region was already investigated by Anderson \& Davis [24]. They found conditions under which Moffatt eddies can be present in viscous flow of two fluids near a corner of two rigid planes. The present work is a variation of this problem, where there are two free surfaces and one of the liquids evaporates. A parametric study is performed to investigate how much flow is generated in the liquid subsphase by the evaporation of the liquid drop as a function of the angles $\theta, \alpha$, and $\beta$, and the viscosity ratio of the two liquids. We first treat the three flow contributions separately, and then identify the regime in which each contribution is asymptotically dominant. 


\subsection{Problem formulation}

The geometry of the drop close to the contact line can be approximated by a twodimensional wedge, see Fig. 4.1(b). The three contact angles between the liquids in the wedge add to $\theta+\alpha+\beta=2 \pi$. To ensure both horizontal and vertical balances between the surface tensions at the three-phase contact line, these angles are restricted to $\theta, \alpha, \beta<\pi$. We adopt a polar coordinate system $(\rho, \phi)$ with the origin located at the contact line and define the velocities in terms of streamfunctions $\Psi_{1}(\rho, \phi)$ and $\Psi_{2}(\rho, \phi)$ as

$$
\begin{array}{ll}
u_{\rho_{1}}(\rho, \phi)=-\frac{1}{\rho} \frac{\partial \Psi_{1}}{\partial \phi}, & u_{\phi_{1}}(\rho, \phi)=\frac{\partial \Psi_{1}}{\partial \rho}, \\
u_{\rho_{2}}(\rho, \phi)=-\frac{1}{\rho} \frac{\partial \Psi_{2}}{\partial \phi}, & u_{\phi_{2}}(\rho, \phi)=\frac{\partial \Psi_{2}}{\partial \rho} .
\end{array}
$$

The flow is governed by the Stokes equations, or equivalently, in terms of the streamfunctions, by the biharmonic equations

$$
\nabla^{4} \Psi_{1}=0, \quad \nabla^{4} \Psi_{2}=0 .
$$

The general self-similar solutions to the biharmonic equations in the two liquids are [25]

$$
\begin{aligned}
& \Psi_{1}(\rho, \phi)=\rho^{m}\left[a_{1} \cos m \phi+a_{2} \sin m \phi+a_{3} \cos (m-2) \phi+a_{4} \sin (m-2) \phi\right], \\
& \Psi_{2}(\rho, \phi)=\rho^{n}\left[c_{1} \cos n \phi+c_{2} \sin n \phi+c_{3} \cos (n-2) \phi+c_{4} \sin (n-2) \phi\right],
\end{aligned}
$$

where $m, n \neq 0,1,2$; in that case, the form (4.3) is degenerate and additional terms arise. The exponents $m$ and $n$ will be selected by the boundary conditions.

The solution to the flow field described by (4.2) consists of three contributions [21] (see Chapter 3). One contribution comes from the evaporative mass flux from the free surface of liquid 1, which drives a flow inside the drop. A second contribution arises from the volume change of the drop due to the evaporative mass loss. In case the contact line of the drop is pinned, there will be a corresponding contribution to the flow field due to the downward motion of the liquid-air interface [21]. On a liquid subphase however, the contact line of the drop is not pinned. Instead, there is a receding motion of the contact line, which results in a decrease in the drop radius and gives a contribution to the flow field. The third contribution to the flow field is the classical "eigenmode" solution to the homogeneous problem for which all boundary conditions are zero, as described by Dean \& Montagnon [26], Moffatt [22], and Anderson \& Davis [24]. From now on, we will refer to these contributions as the flux 
solution, the moving contact-line solution, and the eigenmode solution. Since (4.2) is a linear system, we can consider each of these three contributions separately. The full solution can be obtained by superposition.

\subsubsection{Flux solution}

For the evaporative-flux driven flow, we impose as boundary conditions that liquids 1 and 2 are immiscible and the geometry is fixed. At the interface between liquids 1 and 2 , located at $\phi=0$ (or $\phi=2 \pi)$ this gives

$$
\begin{array}{cc}
u_{\phi_{1}}(\rho, 0) & =0, \\
u_{\phi_{2}}(\rho, 2 \pi) & =0 .
\end{array}
$$

The liquid-air interfaces, located at $\phi=\theta$ (liquid 1) and $\phi=\theta+\alpha$ (liquid 2), are shear-stress free

$$
\begin{array}{cc}
\tau_{\rho \phi_{1}}(\rho, \theta) & =0 \\
\tau_{\rho \phi_{2}}(\rho, \theta+\alpha) & =0
\end{array}
$$

The coupling between liquids 1 and 2, at the boundary $\phi=0$, is made by the continuity of velocity and shear stress conditions at the liquid-liquid interface,

$$
\begin{aligned}
u_{\rho_{1}}(\rho, 0) & =u_{\rho_{2}}(\rho, 2 \pi), \\
\tau_{\rho \phi_{1}}(\rho, 0) & =\tau_{\rho \phi_{2}}(\rho, 2 \pi) \\
\text { or } \eta_{1}\left[\rho \frac{\partial}{\partial \rho}\left(\frac{u_{\phi_{1}}}{\rho}\right)+\frac{1}{\rho} \frac{\partial u_{\rho_{1}}}{\partial \phi}\right]_{\phi=0} & =\eta_{2}\left[\rho \frac{\partial}{\partial \rho}\left(\frac{u_{\phi_{2}}}{\rho}\right)+\frac{1}{\rho} \frac{\partial u_{\rho_{2}}}{\partial \phi}\right]_{\phi=2 \pi},
\end{aligned}
$$

with $\eta_{1}$ and $\eta_{2}$ the dynamic viscosities of liquids 1 and 2, respectively. As a consequence, the viscosity ratio $\eta=\eta_{1} / \eta_{2}$ will appear as a parameter in the solution. The conditions above are the homogeneous boundary conditions for the coupled system of $\Psi_{1}$ and $\Psi_{2}$. The inhomogeneous condition comes from the evaporative flux that drives the flow. We impose that liquid 1 evaporates, while liquid 2 is assumed non-volatile

$$
\begin{aligned}
u_{\phi_{1}}(\rho, \theta) & =\frac{1}{\rho_{\ell}} J(\rho), \\
u_{\phi_{2}}(\rho, \alpha+\theta) & =0,
\end{aligned}
$$

with $J$ the evaporative flux and $\rho_{\ell}$ the liquid density. The evaporative flux from the drop surface is known from the solution to the diffusion equation describing the vapor 
concentration field in a corner geometry [1]

$$
\frac{J(\rho)}{\rho_{\ell}}=A(\alpha) U \rho^{\lambda(\alpha)-1} .
$$

The prefactor $A$ can be obtained from the asymptotics of the full spherical-cap solution (Popov [13]), and $\lambda(\alpha)=\pi / 2 \alpha$. Here $U=D \Delta c / R \rho_{\ell}$ is the velocity scale, which is of order $\mu \mathrm{m} / \mathrm{s}$ for water drops under atmospheric conditions [3], with $R$ the radius of the drop as indicated in Fig. 4.1(a), $D$ the diffusion constant for vapor in air, and $\Delta c=c_{s}-c_{\infty}$ the vapor concentration difference (in $\mathrm{kg} / \mathrm{m}^{3}$ ) between the drop surface and the surroundings. Hence, for $\alpha>\pi / 2$ the evaporative flux diverges as the contact line is approached, while for $\alpha<\pi / 2$ the evaporation is suppressed near the contact line.

The eight boundary conditions for the biharmonic equations (4.2) are thus given by (4.4-4.7). The inhomogeneous evaporative flux condition (4.7a) is driving the flow in liquid 1 , which in turn generates a flow in liquid 2 via the coupling conditions (4.6). From condition (4.7a) we find that exponent in (4.3a) is $m=\lambda(\alpha)$. The exponent in (4.3b) is selected by the coupling condition (4.6a), which implies $n=m=\lambda(\alpha)$. The coefficients $a_{1}$ to $c_{4}$ in (4.3a) and (4.3b) can be found from the boundary conditions (4.4)-(4.7). The system of equations that has to be solved to find these coefficients is

$$
\mathrm{Cx}=\mathbf{b},
$$

with

$$
\mathbf{x}=\left(a_{1}, a_{2}, a_{3}, a_{4}, c_{1}, c_{2}, c_{3}, c_{4}\right),
$$

a vector containing the coefficients $a_{1}$ to $c_{4}$. The right-hand side vector $\mathbf{b}$ contains only zeros except for element $b_{7}=A U$, which comes from the evaporative driving term. The coefficient matrix $C$ is given by

$$
\mathrm{C}=\left(\begin{array}{cccccccc}
1 & 0 & 1 & 0 & 0 & 0 & 0 & 0 \\
0 & 0 & 0 & 0 & C_{1} & S_{1} & C_{1} & S_{1} \\
\lambda C_{2} & \lambda S_{2} & (\lambda-2) C_{3} & (\lambda-2) S_{3} & 0 & 0 & 0 & 0 \\
0 & 0 & 0 & 0 & \lambda C_{4} & \lambda S_{4} & (\lambda-2) C_{5} & (\lambda-2) S_{5} \\
0 & -\lambda & 0 & -(\lambda-2) & -\lambda S_{1} & \lambda C_{1} & -(\lambda-2) S_{1} & (\lambda-2) C_{1} \\
\eta \lambda & 0 & \eta(\lambda-2) & 0 & -\lambda C_{1} & -\lambda S_{1} & -(\lambda-2) C_{1} & -(\lambda-2) S_{1} \\
\lambda C_{2} & \lambda S_{2} & \lambda C_{3} & \lambda S_{3} & 0 & 0 & 0 & 0 \\
0 & 0 & 0 & 0 & C_{4} & S_{4} & C_{5} & S_{5}
\end{array}\right)
$$

with

$$
\begin{aligned}
& C_{1}=\cos 2 \pi \lambda, \quad C_{2}=\cos \lambda \theta, \quad C_{3}=\cos (\lambda-2) \theta \\
& C_{4}=\cos \lambda(\alpha+\theta), \quad C_{5}=\cos (\lambda-2)(\alpha+\theta), \\
& S_{1}=\sin 2 \pi \lambda, \quad S_{2}=\sin \lambda \theta, \quad S_{3}=\sin (\lambda-2) \theta \\
& S_{4}=\sin \lambda(\alpha+\theta), \quad S_{5}=\sin (\lambda-2)(\alpha+\theta) .
\end{aligned}
$$


(a)

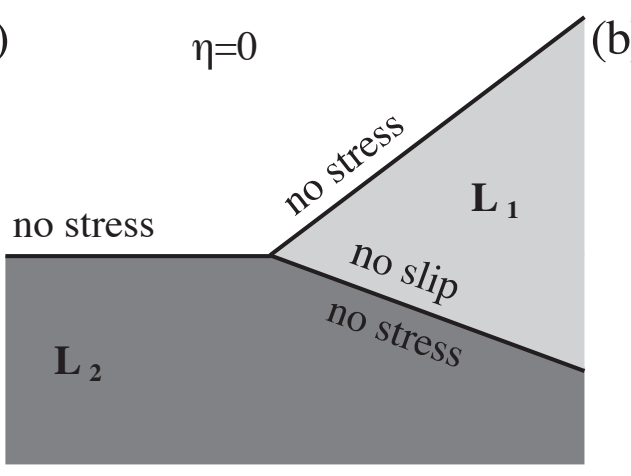

(b)

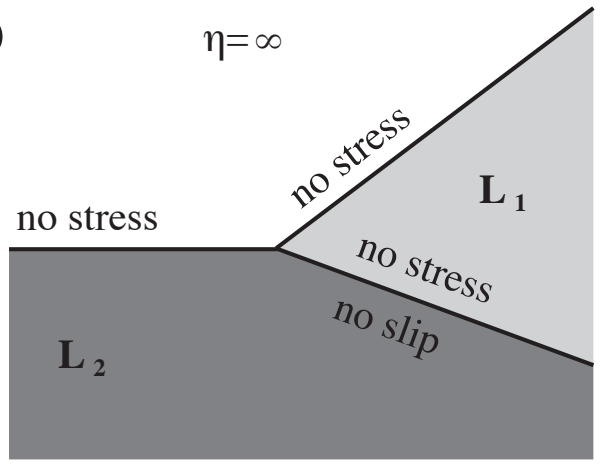

Figure 4.2: Schematic of the boundary conditions experienced by liquids 1 and 2 in the two limiting cases. (a) $\eta=0$ : Liquid 1 experiences a no-stress condition at the free surface and a no-slip condition at the interface with liquid 2, whereas liquid 2 experiences two no-stress conditions. (b) $\eta \rightarrow \infty$ : Liquid 1 experiences two nostress conditions whereas liquid 2 obeys a no-stress condition at the free surface and a no-slip condition at the interface with liquid 1 .

A solution to (4.9) can be found when $\operatorname{det}(C) \neq 0$. Critical points in the solution to (4.9) appear for those values of $\alpha, \theta, \eta$ where $\operatorname{det}(\mathrm{C})=0$. In these cases the eight boundary conditions are no longer linearly independent and the eigenmode solution comes into play [21], as will be discussed in Section 4.2.3.

The $8 \times 8$ matrix given by (4.11) describes the coupled flow in the two liquids. We anticipate that a decoupling into two $4 \times 4$ systems arises for $\eta=0$ and for $\eta \rightarrow$ $\infty$. In these limiting cases, the flows in the two liquids are completely independent. The boundary conditions experienced by each of the two liquids in these decoupled cases are sketched in Fig. 4.2. For $\eta=0$, liquid 1 effectively experiences a no-slip condition at the interface with liquid 2 , as if it were contacting a solid substrate. This feature is due to the extremely high viscosity of the liquid subphase. For $\eta \rightarrow \infty$, the stresses in liquid 2 are so small that effectively a no-stress condition applies and the interface is a free surface. For liquid 2 it is exactly the other way around: no-stress at the interface with liquid 1 when $\eta=0$ and no-slip when $\eta \rightarrow \infty$.

\subsubsection{Moving contact-line solution}

As the drop evaporates its volume decreases and the contact line recedes. The resulting translation of the interface of liquid 1 gives rise to kinematic boundary conditions at the interface between liquids 1 and 2 (located at $\phi=0$ ) and the free surface of 
liquid 1 (located at $\phi=\theta$ )

$$
\begin{aligned}
& u_{\phi_{1}}(\rho, 0)=\frac{d R}{d t} \sin (\alpha+\theta)=u_{\phi_{2}}(\rho, 2 \pi) \\
& u_{\phi_{1}}(\rho, \theta)=\frac{d R}{d t} \sin \alpha
\end{aligned}
$$

with $R(t)$ the radius of the drop as indicated in Fig. 4.1(a), and $d R / d t$ the contact-line speed. We solve the biharmonic equations describing the flows in both liquids subject to the boundary conditions (4.13) and (4.5-4.6), (4.7b). Since (4.13a) and (4.13b) are independent of $\rho$, we obtain for the exponents in (4.3) $m=n=1$. For $m, n=1$, the solutions (4.3a) and (4.3b) are degenerate, and the solutions to the biharmonic equations are of the form [22]

$$
\begin{aligned}
& \Psi_{1}(\rho, \phi)=\rho\left[a_{1} \cos \phi+a_{2} \sin \phi+a_{3} \phi \cos \phi+a_{4} \phi \sin \phi\right], \\
& \Psi_{2}(\rho, \phi)=\rho\left[c_{1} \cos \phi+c_{2} \sin \phi+c_{3} \phi \cos \phi+c_{4} \phi \sin \phi\right] .
\end{aligned}
$$

By solving for the coefficients in (4.14a) and (4.14b) using boundary conditions (4.13) and (4.5-4.6), (4.7b), we obtain

$$
\Psi_{1}(\rho, \phi)=\Psi_{2}(\rho, \phi)=\frac{d R}{d t} \rho \sin (\alpha+\theta-\phi) .
$$

This result is simply a uniform flow away from the contact line.

To find an expression for the contact-line speed $d R / d t$ we can again use the solution to the evaporative outer problem [13]

$$
\frac{d V}{d t}=-\pi R \frac{D \Delta c}{\rho_{\ell}} f(\alpha),
$$

with $V$ the drop volume. An expression for $f$ can be obtained from the integrated evaporative flux from a spherical cap and is given by [16] (see Chapter 2)

$$
f(\alpha)=\frac{\sin (\pi-\alpha)}{1+\cos (\pi-\alpha)}+4 \int_{0}^{\infty} \frac{1+\cosh 2(\pi-\alpha) \tau}{\sinh 2 \pi \tau} \tanh (\alpha \tau) d \tau
$$

In contrast to the model of Popov [13], the drop geometry on a liquid subphase consists of two spherical caps instead of one. In this case, the drop volume is given by

$$
\begin{aligned}
V(\alpha, \theta) & =\pi R^{3}\left[\frac{-\cos ^{3}(\alpha)+3 \cos (\alpha)+2}{3 \sin ^{3} \alpha}+\frac{\cos ^{3}(\alpha+\theta)-3 \cos (\alpha+\theta)-2}{3 \sin ^{3}(\alpha+\theta)}\right] \\
& =\pi R^{3} h(\alpha, \theta) .
\end{aligned}
$$


Assuming that contact angles $\alpha$ and $\theta$ remain constant as the contact line recedes, we obtain an equation for the rate of change of the drop radius

$$
\frac{d R}{d t}=-\frac{1}{3} U \frac{f(\alpha)}{h(\alpha, \theta)}
$$

where $U=D \Delta c / R \rho_{\ell}$ is again the characteristic velocity, which is known for a given drop geometry. From (4.19) we obtain a prediction for the evolution of the drop radius in time that could be checked experimentally

$$
R(t)=\sqrt{\frac{2 D \Delta c f(\alpha)}{3 \rho_{\ell} h(\alpha, \theta)}\left(t_{f}-t\right)},
$$

with $t_{f}$ the total evaporation time of the drop.

\subsubsection{Eigenmode solution}

The eigenmode solution is the nontrivial solution to the homogeneous problem [22]. The form of the solution is still given by (4.3a) and (4.3b), but the exponents $m$ and $n$ are selected by the criterion $\operatorname{det}(C)=0$, with $C$ given by (4.11). In case the determinant of the coefficient matrix $C$ is equal to zero, the system of equations is degenerate and one of the equations becomes redundant. In practise, one can find the coefficients of the eigenmode flow by setting one coefficient equal to an arbitrary value (here taken unity) and removing one line from the coefficient matrix (4.11) to remove the redundancy. The last column of the coefficient matrix then serves as the right-hand side vector. This procedure implies that the strength of the eigenmode flow cannot be determined from the "inner" Stokes flow problem close to the contact line, but is set by the outer flow in the drop [21,27].

For the standard case of a drop evaporating from a flat solid substrate, the condition for the (Moffatt) eigenmode solution was found to be [21] (see Chapter 3)

$$
M\left(\lambda_{E}, \theta\right)=\sin 2\left(\lambda_{E}-1\right) \theta-\left(\lambda_{E}-1\right) \sin 2 \theta=0,
$$

where $\lambda_{E}$ is the exponent of the eigenmode solution. To ensure regularity of the velocity field at the origin, only the roots that have $\operatorname{Re}\left(\lambda_{E}\right)>1$ are considered. The case $\lambda_{E}=2$ is a trivial root and does not correspond to an eigenmode. The present case of a drop evaporating from a liquid subphase is more general, since the substrate is a liquid that is not flat. The limit of $\eta=0$ effectively corresponds to a liquid evaporating from a solid substrate, and in this $\operatorname{case} \operatorname{det}(\mathrm{C})$ reduces to

$$
\operatorname{det}(\mathrm{C})=F\left(\alpha, \lambda_{E}\right) M\left(\lambda_{E}, \theta\right)
$$


where

$$
F(\alpha, \theta)=-8 \lambda \sin \{\lambda[2 \pi-(\alpha+\theta)]\} \sin \{\lambda[2 \pi-(\alpha+\theta)]+2(\alpha+\theta)\} .
$$

When $\alpha=\pi-\theta, F=-8 \lambda \sin ^{2}(\pi \lambda)$ and we exactly recover the case of a liquid evaporating from a flat solid substrate [21] given by (4.21). For arbitrary $\eta$ and $\alpha$ the equation $\operatorname{det}(\mathrm{C})=0$ gives the solution to the generalized corner-flow problem of a liquid on a liquid subphase, where, instead of a no-slip condition, a coupling condition between the two liquids applies. Hence, the exponent of the eigenmode solution is a function of the three problem parameters: $m=n=\lambda_{E}(\alpha, \theta, \eta)$. This problem is a variation of the one studied by Anderson \& Davis [24], where a twodimensional viscous flow of two fluids in the corner between two solid walls was studied. Here, instead of two solid walls there are two free surfaces.

\subsection{Results}

\subsubsection{Flux solution}

The flows in the evaporating drop and the liquid subphase depend on the angles between the phases $\alpha, \theta$, and $\beta$, and the viscosity ratio $\eta$. To illustrate the effect of these parameters on the velocity fields in both liquids, we show some representative cases. To start we extend our previous work where we studied the flow inside a liquid evaporating from a solid substrate [21] (Chapter 3). The current problem is more general, but will tend to the flat solid case when $\eta \ll 1$, such that liquid 1 experiences a no-slip condition at the interface with liquid 2 , and $\alpha=\pi-\theta$, such that the interface of liquid 2 is flat. In Fig. 4.3(a) we show that in this limit we indeed recover the same flow patterns as described in [21], with the flow in liquid 1 being much stronger than the flow in the more viscous liquid 2. Similarly to the results obtained in our previous work, we observe that for larger contact angles $\theta$ (and hence smaller $\alpha$ ) the flow shows a sequence of reversal structures. When $\theta=50^{\circ}\left(\alpha=130^{\circ}\right)$, the flow is separated into two regions, such that near the interface with liquid 2 , the flow in liquid 1 is directed away from the contact line, towards the center of the drop. The dashed line is the separating flow line that ends in a stagnation point at the contact line. The smaller the angle $\alpha$, the more separatrices appear. Very little flow is generated in the liquid subphase, which is very viscous in the limit $\eta \ll 1$. The influence of the liquid subphase becomes apparent when the viscosity ratio $\eta$ is increased, see Fig. 4.3(b). When the viscosity of liquid 2 becomes comparable to or smaller than the viscosity of liquid 1, the evaporation of liquid 1 generates a flow in liquid 2 via the coupling conditions (4.6a) and (4.6b). The change in viscosity ratio also affects the flow in 
(a)

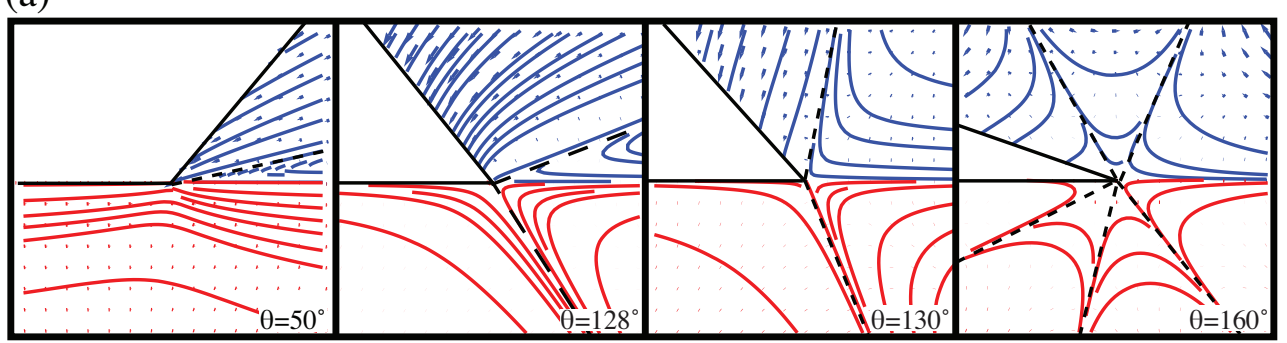

(b)

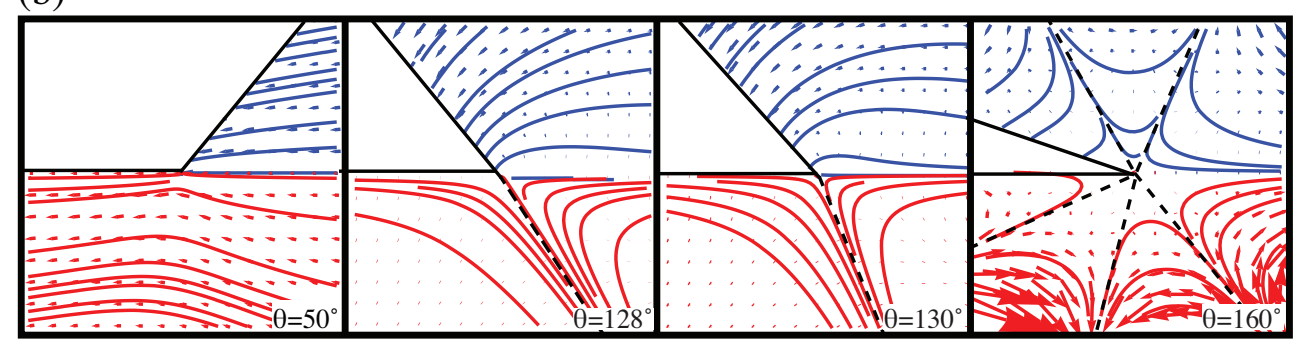

Figure 4.3: (a) Streamline plot of the flux solutions $\Psi_{1}$ (blue) and $\Psi_{2}$ (red) for a viscosity ratio $\eta=1 / 10$ (liquid 2 much more viscous than liquid 1 ). Contact angle $\theta$ is varied from $50^{\circ}$ to $160^{\circ}$, whereas $\alpha$ is adjusted such that the interface of liquid 2 is flat: $\alpha=180^{\circ}-\theta$. The dashed lines represent the separatrices between the different flow regimes. (b) Same as in (a) but with a viscosity ratio $\eta=10$, which means that liquid 1 is much more viscous than liquid 2.

liquid 1, which now experiences a different boundary condition at the liquid-liquid interface. This results in a disappearance of the separatrices in liquid 1 for $\theta=50^{\circ}$, $128^{\circ}$, and $130^{\circ}$.

Note that the cases shown in Fig. 4.3 are somewhat artificial, since a liquid subphase would not remain flat: the Neumann condition at the contact line will require that $\alpha+\theta>180^{\circ}$, to ensure a vertical force balance. To explore a physically more realistic case, we now consider the situation where all surface tensions are equal, for which $\theta=\alpha=\beta=120^{\circ}$. In Fig. 4.4 we plot the resulting streamline patterns for three viscosity ratios $\eta$. It can be observed that the velocity field in liquid 1 nearly obeys a no-slip condition for $\eta=1 / 10$, which means that there is almost no evaporation-driven flow in liquid 2 . When the viscosity ratio increases, the evaporation of liquid 1 generates more flow in liquid 2, which can be seen from the larger arrow size in Fig. 4.4 for $\eta=10$ compared to $\eta=1 / 10$. For $\eta=10$, the flow in liquid 1 follows an almost free-slip condition. The strength of the flow generated in 


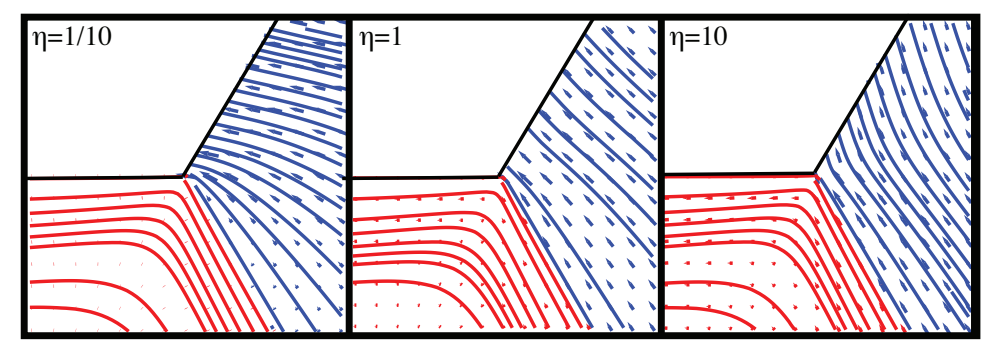

Figure 4.4: Streamline plot of the flux solutions $\Psi_{1}$ (blue) and $\Psi_{2}$ (red) for $\theta=\alpha=$ $\beta=120^{\circ}$, which means all surface tensions are equal. The viscosity ratio is varied from $\eta=1 / 10$ (liquid 1 experiences almost no slip at the interface with liquid 2) to $\eta=10$ (almost free slip).

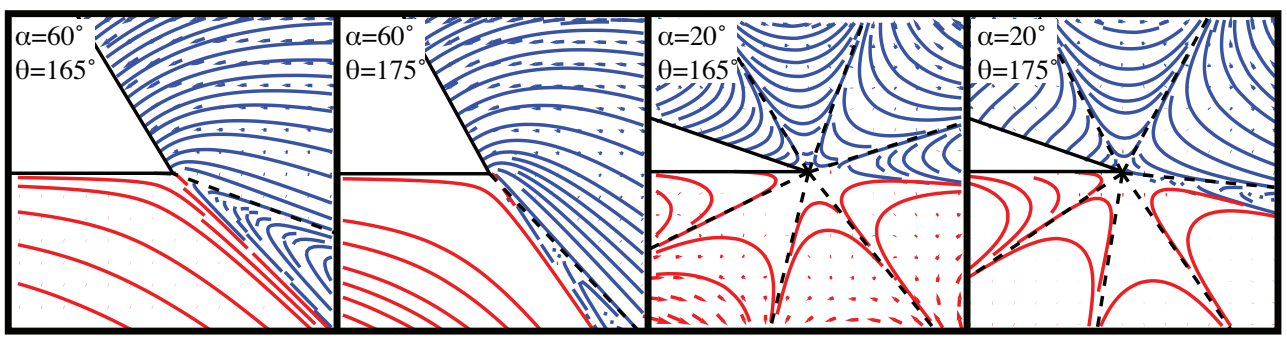

Figure 4.5: Streamline plot of the flux solutions $\Psi_{1}$ (blue) and $\Psi_{2}$ (red) for $\alpha=60^{\circ}$ and $\alpha=20^{\circ}$, where $\theta$ is varied from $165^{\circ}$ to $175^{\circ}$, for $\eta=10$. For small $\alpha$, a sequence of wedge-like flow structures is observed in both phases.

the liquid subphase as a function of the viscosity ratio will be analysed further below.

In Fig. 4.4 we do not see the reversing flow structures we observed in Fig. 4.3. The reason for this feature is that the sequences of wedge-like flow structures shown in Fig. 4.3 are triggered by large exponents $\lambda$. We can understand this behaviour by inspecting the self-similar expressions for the streamfunctions given by (4.3): since $m=n=\lambda$, a high frequency in the $\phi$-direction arises when $\lambda \gg 1$. Hence, when $\alpha \ll 90^{\circ}$, which means $\lambda \gg 1$, multiple wedges appear. To illustrate this behaviour we show the flow structures for large and small $\alpha$ in Fig. 4.5. For $\alpha=60^{\circ}$, only one separatrix is present in the flow in liquid 1 . When $\alpha$ is decreased to $20^{\circ}$ however, we obtain a sequence of four wedges, which implies that the flow is frequently changing directions.

We have seen that the evaporation of liquid 1 generates a flow in liquid 2 through 

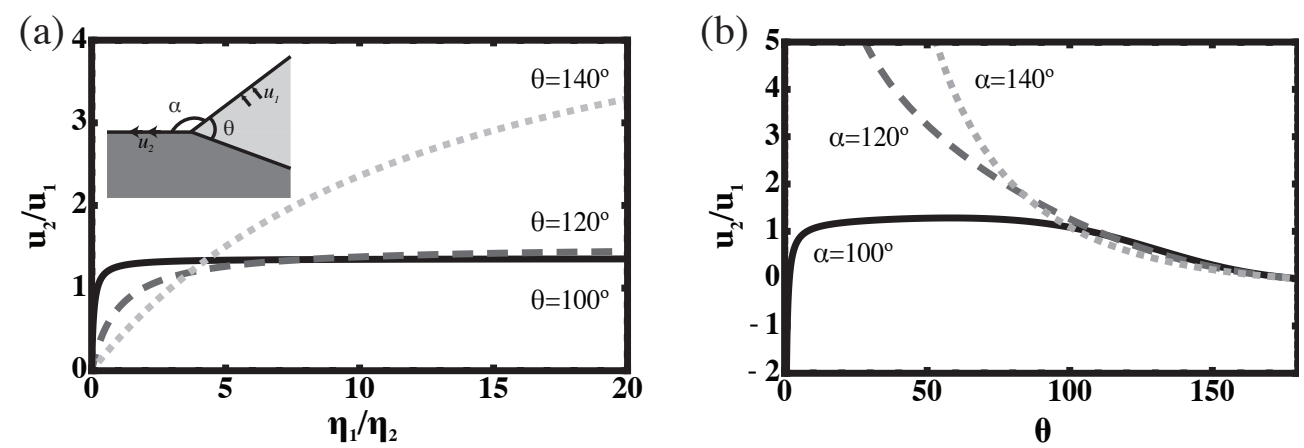

Figure 4.6: (a) Plot of the flow generated at the free surface of liquid 2 compared to the driving by the evaporative flux, $u_{2} / u_{1}$, as a function of the viscosity ratio. The inset shows the definition of $u_{1} \equiv u_{\phi_{1}}(\phi=\theta)$, the velocity towards the free surface of liquid 1 , and $u_{2} \equiv u_{\rho_{2}}(\phi=\alpha+\theta)$, the velocity along the free surface of liquid 2. Here, $\alpha=120^{\circ}$, whereas $\theta$ is varied from $100^{\circ}$ (black, solid line) to $120^{\circ}$ (gray, dashed line) to $140^{\circ}$ (light gray, dotted line). (b) Same velocity ratio as in (a) but now as a function of $\theta$. Parameter $\eta$ is fixed to 1 , whereas $\alpha$ is varied from $100^{\circ}$ (black, solid line), to $120^{\circ}$ (gray, dashed line) to $140^{\circ}$ (light gray, dotted line).

boundary conditions (4.6a) and (4.6b). The strength of the flow generated in liquid 2 depends on the viscosity ratio, as shown in Fig. 4.3 and 4.4. A measure for the amount of flow generated in liquid 2 by the evaporation of liquid 1 is the ratio $u_{\rho_{2}}(\phi=$ $\alpha+\theta) / u_{\phi_{1}}(\phi=\theta)$. This ratio relates the amount of flow generated at the free surface of liquid 2 to the driving term, which is the flow towards the free surface of liquid 1, see the inset in Fig. 4.6(a). Fig. 4.6 shows how this velocity ratio depends on the parameters $\eta, \theta$, and $\alpha$. In Fig. 4.6(a) we observe that when $\eta=0$, the flow in liquid 1 obeys a no-slip condition at the interface between the two liquids, which means no flow is generated in liquid 2. In the opposite limit $\eta \rightarrow \infty$ a free-slip condition applies, with a maximum amount of flow generated in liquid 2. The flow strength in liquid 2 depends on the viscosity ratio between the two liquids, and also on the contact angles $\theta$ and $\alpha$. In Fig. 4.6(b) it is shown that not only the magnitude but also the flow direction depends on $\theta$ and $\alpha: u_{2} / u_{1}$ can change sign. This sign change is due to the appearance of separatrices in the velocity field, as observed in Fig. 4.5.

\subsubsection{Moving contact-line solution}

The moving contact-line solution is just a uniform flow away from the contact line, as given by (4.15). The corresponding flow pattern is shown in Fig. 4.7. No gradients 


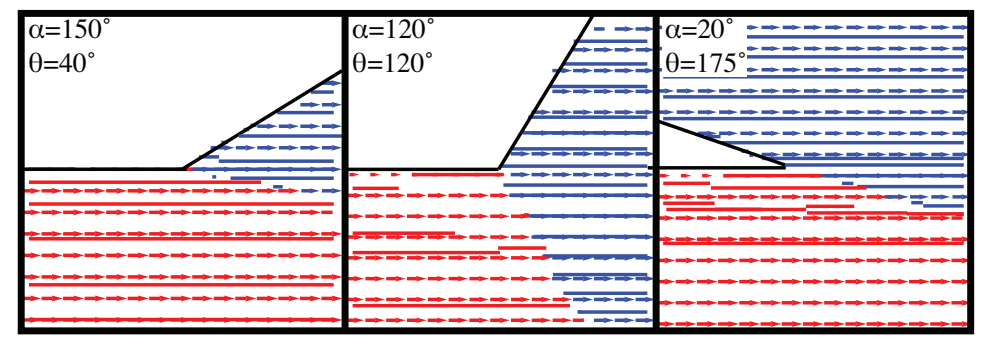

Figure 4.7: Streamline plot of the moving contact-line solutions $\Psi_{1}$ (blue) and $\Psi_{2}$ (red) for various $\alpha, \theta$, and a viscosity ratio $\eta=1$.

in the velocity fields in the two liquids are observed, which means there is no viscous stress. By contrast, the hinge contribution to the velocity field in a pinned evaporating drop on a solid substrate leads to a significant viscous stress, due to the pinning of the contact line and the no-slip condition at the liquid-solid interface [21]. As the exponents of the streamfunctions for the moving contact-line solution are constant, the flow patterns shown in Fig. 4.7 are the same for all angles $\alpha$ and $\theta$ and viscosity ratios $\eta$. While the exponent of the moving contact-line solution is equal to unity, the exponent $\lambda$ for the flux solution is larger than unity when $\alpha<\pi / 2$. Therefore, we anticipate that the moving contact-line solution will be the dominant motion near the contact line when $\alpha<\pi / 2$.

\subsubsection{Eigenmode solution}

The streamline patterns of the eigenmode solution are shown in Fig. 4.8. In the plots, we took $\eta=1$, such that the eigenmodes in liquids 1 and 2 are coupled via the boundary conditions (4.6a) and (4.6b). We observe that the velocity field in liquid 1 is directed inwards along the interface with liquid 2, and outwards along the free surface. The same flow structure applies to liquid 2, however, for $\alpha=20^{\circ}$ a reversal in the flow direction in liquid 2 is observed. In that case, the liquid-liquid interface acts as a separatrix. For the parameters values used in the plots in Fig. 4.8 the exponents of the eigenmode solutions are real-valued, which means no viscous eddies are present [22].

The exponents $\lambda_{E}$ of the eigenmode solution follow from the solution to $\operatorname{det}(\mathrm{C})=0$ and show multiple branches as a function of $\theta, \alpha$, and $\eta$. Asymptotically, the lowest branch that has $\operatorname{Re}\left(\lambda_{E}\right)>1$ (to ensure regularity of the velocity field at the origin) dominates. The trivial roots $\lambda_{E}=1$ and $\lambda_{E}=2$ have to be excluded, as they do not represent an eigenmode, but are special cases for which the form (4.3) is degenerate. 


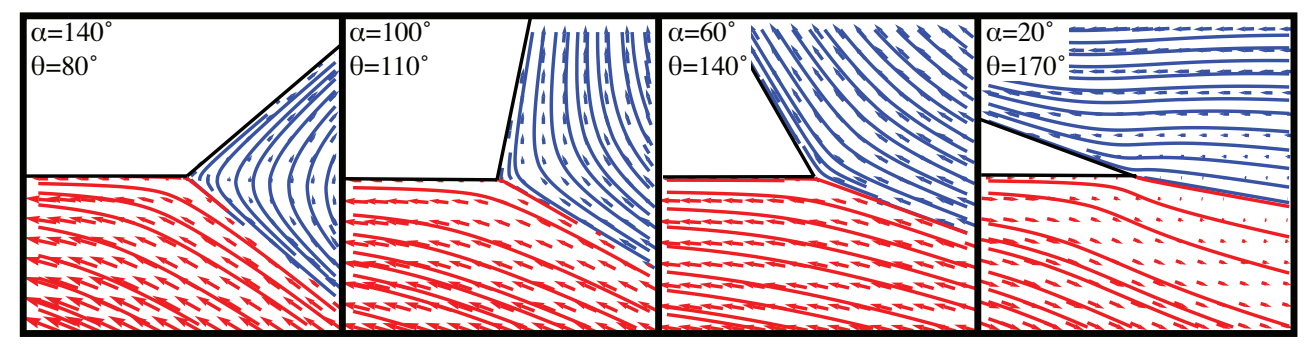

Figure 4.8: Streamline plot of the eigenmode solutions $\Psi_{1}$ (blue) and $\Psi_{2}$ (red) for decreasing $\alpha$ and increasing $\theta$, for a viscosity ratio $\eta=1$.

To understand the structure of the eigenvalues, it is insightful to first describe the limiting cases $\eta=0$ and $\eta \rightarrow \infty$ when the eigenmode flows in liquids 1 and 2 are completely decoupled. In these two limiting cases, the leading-order exponents for the flows in both liquids are depicted as a function of $\theta$ in Fig. 4.9(a) and (b), for a given $\alpha=90^{\circ}$. The leading-order exponent of the eigenmode flow in liquid 1 is shown as a black solid line and in liquid 2 as a gray solid line. In the decoupled case, the exponents for the eigenmode flows in liquids 1 and 2 are different. The expressions for the exponents are still relatively simple, see for example (4.21), compared to the case where the flows in liquids 1 and 2 are coupled.

Introducing a weak coupling between the two liquids (dashed lines in Fig. 4.9(a) and (b)) ensures that the flows in both liquids obey the same exponent. This behaviour means that the two separate branches represented by the solid lines in Fig. 4.9(a) and (b) give rise to two coupled eigenmodes. At the intersection point where the two separate branches (the solid lines) merge, a double root arises. Consequently, when the flow in the two liquids is coupled, there is a root-splitting behaviour of the two coupled eigenmodes at this intersection point. A similar behaviour was observed by Anderson \& Davis [24] for a flow of two liquids between two rigid walls. The dominant eigenmode is the one that is closest to the lowest root of the two uncoupled solutions. This leading-order solution is shown as a dashed line in Fig. 4.9.

To show how the viscosity ratio $\eta$ influences the exponent of the eigenmode flow in the coupled case, we plot the exponent of the eigenmode flow as a function of $\theta$ for different values of $\eta$, for a given $\alpha=90^{\circ}$ in Fig. 4.9(c). The black solid line represents the leading-order exponent of the coupled eigenmode flow for $\eta=$ 1/100, which is the same case as depicted in Fig. 4.9(c) as the black dashed line. The opposite case, $\eta=100$, is shown as a black dashed line in both Fig. 4.9(b) and (c). We observe that as the viscosity ratio $\eta$ is increased for $\eta=1 / 100$ to $\eta=100$, the maximum in the exponent becomes a minimum and vice versa. 
(a)

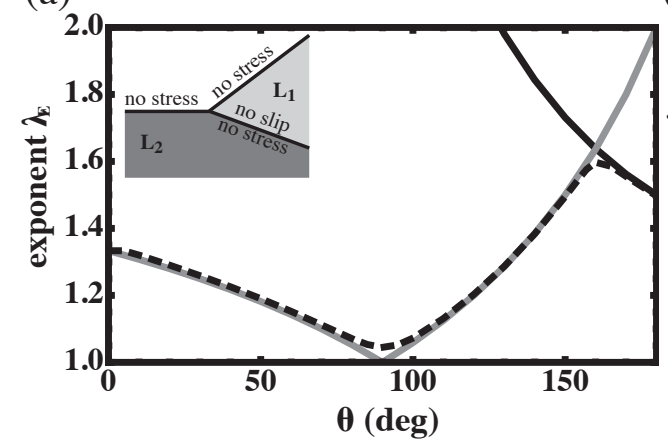

(b)

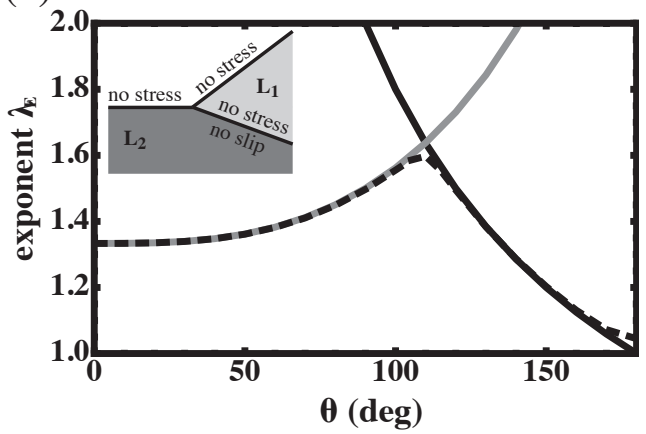

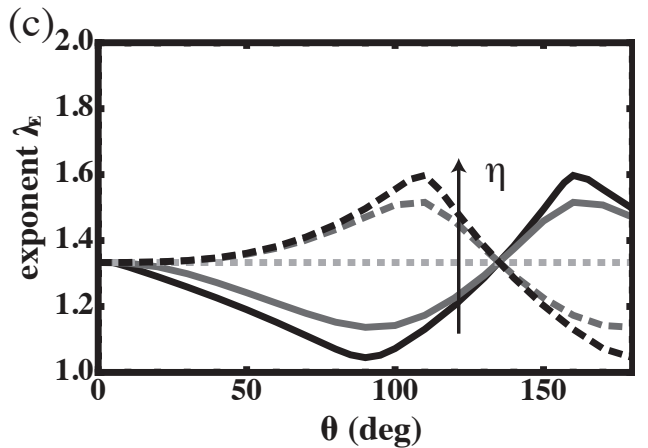

Figure 4.9: (a) Leading-order exponent $\lambda_{E}$ of the eigenmode solution for liquid 1 (black, solid line) and 2 (gray, solid line) as a function of $\theta$ for $\eta=0$, which means the flow in the two liquids is completely decoupled. The inset shows the boundary conditions experienced by liquid 1 and liquid 2 in this case. The black dashed line represents the weakly coupled case where the flow in both liquids obeys the same exponent, for $\eta=1 / 100$. (b) Leading-order exponent of the eigenmode solutions for the decoupled case where $\eta=\infty$. The black dashed line is the weakly coupled case for $\eta=100$. (c) Leading-order exponent of the coupled eigenmode solution for $\eta=1 / 100$ (black, solid line), $\eta=1 / 10$ (gray, solid line), $\eta=1$ (light gray, dotted line), $\eta=10$ (gray, dashed line), and $\eta=100$ (black, dashed line). For all graphs $\alpha=90^{\circ}$. 


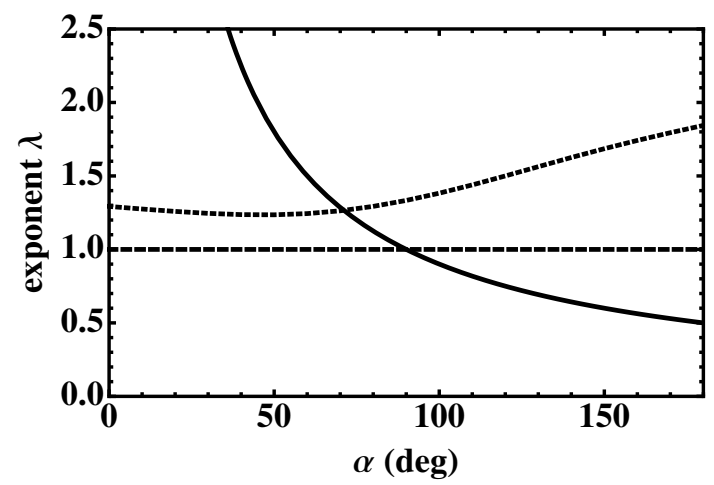

Figure 4.10: Plot of the exponents of the flux (solid line), moving contact line (dashed line), and eigenmode (dotted line) solutions as a function of $\alpha$. For $\alpha<90^{\circ}$ the moving contact-line solution, for which $m=1$, dominates, for $\alpha>90^{\circ}$ the flux solution with $m=\lambda=\pi / 2 \alpha$ (in radians) is dominant. The leading-order exponent of the eigenmode solution, here plotted for $\theta=120^{\circ}$ and $\eta=1$, is always subdominant $\left(m=\lambda_{E}>1\right)$.

\subsubsection{Dominant contribution}

The total evaporation-driven flow in the wedge geometry consists of three contributions: the flux, the moving contact line, and the eigenmode solutions. Each of the three contributions to the total flow in the wedge has a different scaling behaviour with distance to the contact line: all are of the type $\rho^{m}$, but with different $m$. In Fig. 4.10 these exponents are plotted as a function of angle $\alpha$. The moving contact-line solution has exponent $m=1$, which is the smallest of the three exponents for $\alpha<90^{\circ}$. Therefore, the moving contact-line solution dominates the flow near the contact line for $\alpha<90^{\circ}$. The exponent for the flux solution (in radians) is $m=\lambda=\pi / 2 \alpha$. Hence, for $\alpha>90^{\circ}, \lambda<1$ and the flux solution is dominant. The leading-order exponent of the eigenmode solution varies not only with $\alpha$, but also with $\theta$ and $\eta$. Figure 4.10 shows the result for $\theta=120^{\circ}$ and $\eta=1$. However, for all $\theta, \alpha, \eta$ the eigenmode solution is always subdominant, since $m=\lambda_{E}>1$.

\subsection{Discussion}

We have derived self-similar solutions to the velocity field near the contact line of a liquid drop that evaporates from a liquid subphase. The nature of the flow strongly 
depends on the contact angles given by the Neumann construction. In both phases reversing flow structures can be observed, in particular when the angle $\alpha<90^{\circ}$. We found that there are three contributions to the flow in the wedge geometry: one contribution that is driven by the evaporative flux from the drop surface, one contribution from the receding contact line, and one eigenmode contribution. The amplitudes of the flux and moving contact-line solution are known from the full spherical-cap solution for the vapour transport [13]. However, the amplitude of the eigenmode flow can only be obtained by solving the velocity field in the entire drop, which has to be done numerically. Which of the three flow contributions is asymptotically dominant close to the contact line depends on the contact angle $\alpha$ : for $\alpha<90^{\circ}$ we found that the moving contact-line solution dominates, whereas for $\alpha>90^{\circ}$, the flux solution is dominant. The eigenmode solution is always subdominant, in contrast to the drop on a solid substrate, for which the eigenmode solution is the dominant flow for contact angles larger than $133.4^{\circ}$ (see Chapter 3).

A fourth contribution to the flow could come from the Marangoni effect: the nonuniform evaporation of the drop leads to temperature gradients on the drop surface, which induces Marangoni stresses [5]. These Marangoni stresses can give rise to additional flow circulation in the drop, and also alter the velocity field in the liquid subphase. In principle, our analysis could be extended to include the Marangonidriven contribution to the flow in the two liquids following the method of Ristenpart et al. [5].

It would be interesting to see if the reversing flow structures found in the present analysis can be measured in an experiment. A direct consequence of our analysis of the moving contact-line solution is the exact expression (including the prefactor) for the time evolution of the drop radius in time (4.20), which could easily be verified. To our knowledge, the evaporation time of a drop on a liquid subphase has not been investigated experimentally so far.

We have applied the solutions to the flow in a wedge geometry that contains two liquid phases to the situation of a volatile drop evaporating from a non-volatile liquid subphase. The same solutions also hold for the inverse problem of a non-volatile drop on a volatile subphase, such as oil drops on water. In that case, however, the outer problem for the evaporation is different, since we have a volatile liquid bath instead of a volatile drop. Evaporation will lead to a decrease in the bath level, leaving the drop geometry unchanged. Hence, the prefactor in the expression for the evaporative flux (4.8) will be different. Since there is no contact-line motion in this case, also the eigenmode flow can become important. 


\section{References}

[1] R. D. Deegan, O. Bakajin, T. F. Dupont, G. Huber, S. R. Nagel, and T. A. Witten, "Capillary flow as the cause of ring stains from dried liquid drops", Nature 389, 827-828 (1997).

[2] R. D. Deegan, O. Bakajin, T. F. Dupont, G. Huber, S. R. Nagel, and T. A. Witten, "Contact line deposits in an evaporating drop", Phys. Rev. E 62, 756765 (2000).

[3] A. G. Marín, H. Gelderblom, D. Lohse, and J. H. Snoeijer, "Order-to-disorder transition in ring-shaped colloidal stains”, Phys. Rev. Lett. 107, 085502 (2011).

[4] P. J. Yunker, T. Still, M. A. Lohr, and A. G. Yodh, "Suppression of the coffeering effect by shape-dependent capillary interactions", Nature 476, 308-311 (2011).

[5] W. D. Ristenpart, P. G. Kim, C. Domingues, J. Wan, and H. A. Stone, "Influence of substrate conductivity on circulation reversal in evaporating drops", Phys. Rev. Lett. 99, 234502 (2007).

[6] G. J. Dunn, S. K. Wilson, B. R. Duffy, S. David, and K. Sefiane, "The strong influence of substrate conductivity on droplet evaporation", J. Fluid Mech. 623, 329-351 (2009).

[7] K. Sefiane and R. Bennacer, "An expression for droplet evaporation incorporating thermal effects", J. Fluid Mech. 667, 260-271 (2011).

[8] R. W. Style and E. R. Dufresne, "Static wetting on deformable substrates, from liquids to soft solids", Soft Matter 8, 7177 (2012).

[9] A. Marchand, S. Das, J. H. Snoeijer, and B. Andreotti, "Contact angles on a soft solid: from Young's law to Neumann's law”, Phys. Rev. Lett. 109, 236101 (2012).

[10] M. C. Lopes and E. Bonaccurso, "Evaporation control of sessile water drops by soft viscoelastic surfaces", Soft Matter 8, 7875 (2012).

[11] R. W. Style, Y. Che, J. S. Wettlaufer, L. Wilen, and E. R. Dufresne, "Universal deformation of soft substrates near a contact line and the direct measurement of solid surface stresses", to appear in Phys. Rev. Lett. (2012). 
[12] H. Hu and R. G. Larson, "Evaporation of a sessile droplet on a substrate", J. Phys. Chem. B 106, 1334-1344 (2002).

[13] Y. O. Popov, "Evaporative deposition patterns: Spatial dimensions of the deposit”, Phys. Rev. E 71, 036313 (2005).

[14] J. Eggers and L. M. Pismen, "Nonlocal description of evaporating drops", Phys. Fluids 22, 112101 (2010).

[15] A. M. Cazabat and G. Guéna, "Evaporation of macroscopic sessile droplets", Soft Matter 6, 2591-2612 (2010).

[16] H. Gelderblom, A. G. Marín, H. Nair, A. van Housselt, L. Lefferts, J. H. Snoeijer, and D. Lohse, "How water droplets evaporate on a superhydrophic substrate”, Phys. Rev. E 83, 026306 (2011).

[17] B. Sobac and D. Brutin, "Triple-line behavior and wettability controlled by nanocoated substrates: influence on sessile drop evaporation", Langmuir 27, 14999-15007 (2011).

[18] G. Berteloot, C. T. Pham, A. Daerr, F. Lequeux, and L. Limat, "Evaporationinduced flow near a contact line: consequences on coating and contact angle", Europhys. Lett. 83, 14003 (2008).

[19] H. Hu and R. G. Larson, "Analysis of the effects of marangoni stresses on the microflow in an evaporating sessile droplet", Langmuir 21, 3972-3980 (2005).

[20] H. Hu and R. Larson, "Marangoni effect reverses coffee-ring depositions", J. Phys. Chem. B 110, 7090-7094 (2006).

[21] H. Gelderblom, O. Bloemen, and J. H. Snoeijer, "Stokes flow near the contact line of an evaporating drop", J. Fluid Mech. 709, 69-84 (2012).

[22] H. K. Moffatt, "Viscous and resistive eddies near a sharp corner", J. Fluid Mech. 18, 1-18 (1964).

[23] P.-G. de Gennes, F. Brochard-Wyart, and D. Quéré, Capillarity and wetting phenomena (Springer) (2004).

[24] D. M. Anderson and S. H. Davis, "Two-fluid viscous flow in a corner", J. Fluid Mech. 257, 1-31 (1993). 
[25] J. H. Michell, "On the direct determination of stress in an elastic solid, with application to the theory of plates", Proc. London Math. Soc. 100, 100-124 (1899).

[26] W. R. Dean and P. E. Montagnon, "On the steady motion of viscous liquid in a corner”, Proc. Camb. Phil. Soc. 45, 389-394 (1949).

[27] H. K. Moffatt and B. R. Duffy, "Local similarity solutions and their limitations", J. Fluid Mech. 96, 299-313 (1980). 


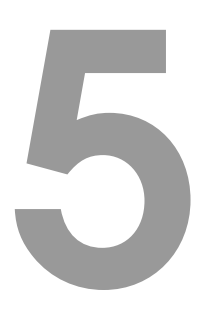

\section{Order-to-disorder transition in ring-shaped colloidal stains $* \dagger$}

In Chapter 3, the fluid flow inside an evaporating drop with a pinned contact line was described. In this chapter, we show that if such an evaporating drop contains colloidal particles, remarkable drying patterns can be formed. A colloidal dispersion drop evaporating from a surface, such as a drying coffee drop, leaves a distinct ring-shaped stain. This phenomenon was explained by the pioneering work of Deegan et al. [1]. Our experiments with monodisperse colloidal particles reveal a structural transition in the stain, from ordered crystals to disordered packings. This sharp transition originates from a temporal singularity of the flow velocity inside the evaporating drop at the end of its life. When the deposition speed is low, particles have time to arrange by Brownian motion, while at the end, high-speed particles are jammed into a disordered phase.

\footnotetext{
*Published as:
}

Álvaro G. Marín, Hanneke Gelderblom, Detlef Lohse, and Jacco H. Snoeijer, “Order-to-disorder transition in ring-shaped colloidal stains”, Phys. Rev. Lett. 107, 085502 (2011).

${ }^{\dagger}$ The experimental work is performed by Álvaro G. Marín. 


\subsection{Introduction}

Ordered arrays of colloidal particles present important characteristics for fields as photonics [2] and biotechnology [3]. A relatively simple approach to deposit particles onto a substrate is by evaporation of a colloidal dispersion drop. In drops with pinned contact lines, evaporation gives rise to the so-called coffee-stain effect $[1,4$ 8]: a ring-like stain remains on the substrate once the liquid has evaporated. The mechanism behind this ring-stain formation is explained in the pioneering work of Deegan et al. [1]: in an evaporating drop with an immobile contact line, a capillary flow is generated to replenish the liquid that has evaporated from the edges, as discussed in Chapter 1. This flow drags particles towards the contact line, forming the ring-shaped stain in particle suspensions such as coffee.

This deposition mechanism has been successfully used to generate colloidal crystals in three dimensions [9]. Furthermore, techniques have been developed to control the patterns by permitting evaporation through specially designed masks [10] or by controlling the wettability $[8,11]$. Also large-scale colloidal crystals have been observed for particle sizes below $100 \mathrm{~nm}$ [12], but some displayed instabilities that gave rise to concentric rings [13], stripes, chevrons [14], and cracks [15]. At present, there is no way of predicting beforehand the structure of the deposit remaining after evaporation, due to a lack of understanding of the basic mechanisms. However, the processes driving colloidal crystallization are crucial for controlling the mechanical and the optical properties of the synthesized materials.

In this chapter, we reveal the conditions leading to colloidal crystallization in a paradigmatic system: a 3- $\mu \mathrm{L}$ sessile water drop containing colloidal particles evaporating from a smooth glass slide (Fig. 5.1(a)). The colloids consist of almost monodisperse red-fluorescent polystyrene particles with particle diameters in the range of 0.5 to $2 \mu \mathrm{m}$. The drop is gently deposited onto a glass, and is left to evaporate under controlled temperature and humidity. The contact line remains pinned during the entire experiment. Our aim is to analyse structure of the particle arrangement in the remaining coffee stain.

\subsection{Analysis of the colloidal stain}

It turns out that the dynamics of particle deposition is crucial for the understanding of the final structure. Therefore, the entire evaporative process is filmed simultaneously from the side and from below (see Appendix A.1). This allows to follow the evolution of the drop geometry, the formation of the deposit, and the dynamics of particle migration. The particles are filmed from below with a CMOS camera (PCO imaging) 

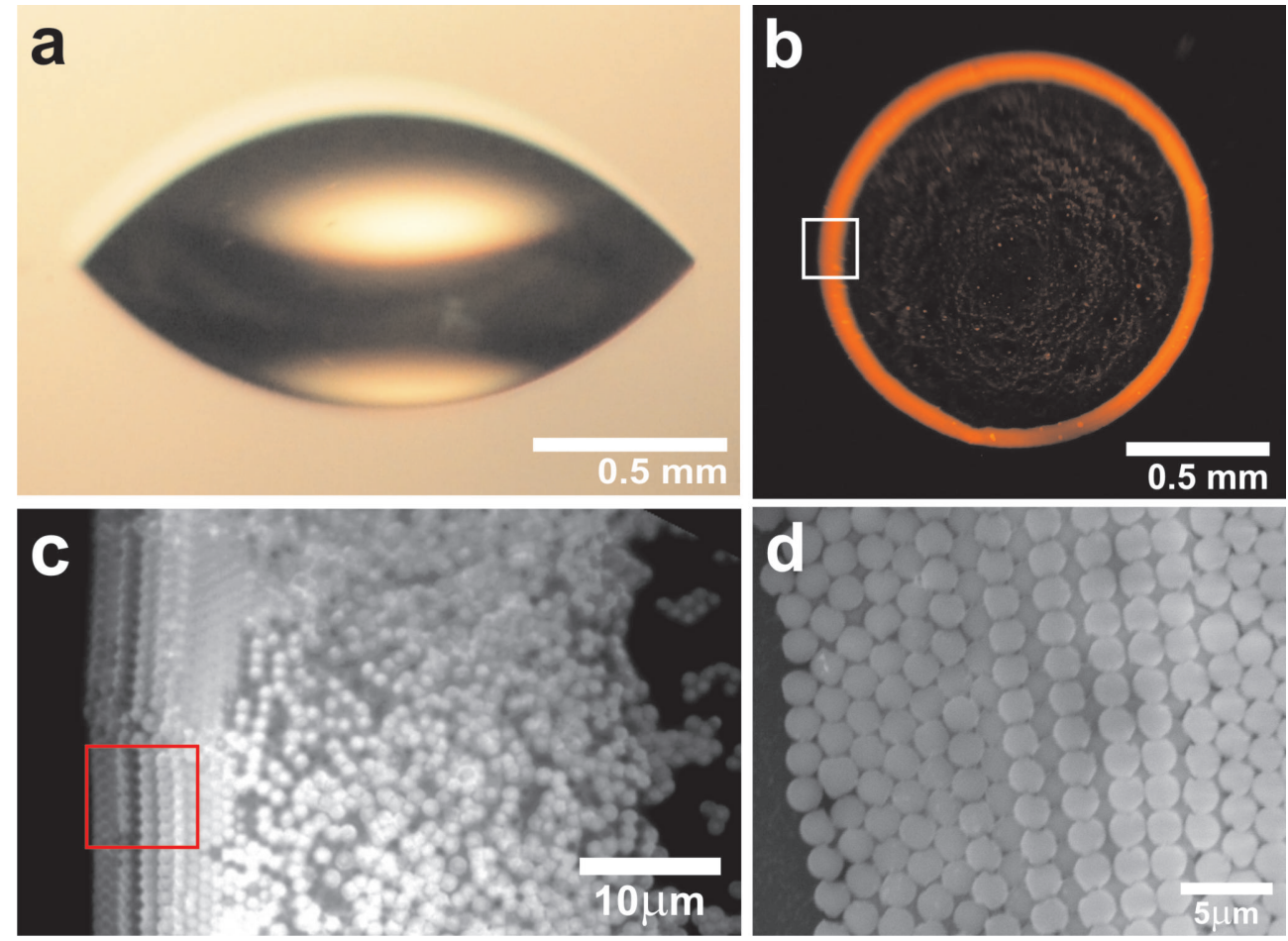

Figure 5.1: Order-to-disorder transition in the particle stain left by an evaporating drop. (a) A 3- $\mu \mathrm{L}$ sessile water drop evaporates from a glass substrate. (b) Ringshaped stain of red particles (the coffee stain) left on the substrate after evaporation. (c) A close-up by an optical microscope of the bottom layer of the stain, taken from the white square in (b), shows that the outermost lines of the stain (left) have an ordered, crystalline structure. Towards the center of the drop (right), a transition to a disordered particle arrangement is observed. (d) A top view of the ring stain, taken from the red square in (c) with the Scanning Electron Microscope (SEM), shows that the first lines of particles (left) are arranged in a hexagonal array, while the following lines (brighter in the image) are arranged as square, followed by again hexagonal array. 
connected to an inverted microscope. An integrated standard halogen light source is used to illuminate the particles; the emitted light is filtered before it reaches the camera. The camera is operated at a typical frame rate of 1 frame per second, which is sufficient to capture the movement of the particles. To determine the velocity field in the measurement area from the captured images, a custom-made MATLAB micro-Particle Image Velocimetry ( $\mu$ PIV) code was used. The side-view images are used to calculate contact angle, volume, radius, and height of the drop at every instant with a custom-made MATLAB code. Side-view images are taken with a long distance microscope and a CCD camera (Lumenera Corp.), synchronized with the visualization of the particles by a common trigger.

When the drop has completely evaporated, most of the colloidal particles have aggregated into a ring-shaped coffee stain (Fig. 5.1(b)). Zooming in on the stain, we see that the particle arrangement is not homogeneous (Fig. 5.1(c)): there is a remarkable transition from a crystalline arrangement to a disordered phase. A top view of the stain (Fig. 5.1(d)) shows a sequence of particle arrangements within the ordered phase, starting from hexagonal packing, followed by square packing, and again hexagonal packing. The particle arrangement is analyzed in more detail in Fig. 5.2. Close to the contact line, we find a square arrangement of particles (Fig. 5.2(b1)), followed by a hexagonal array (Fig. 5.2(b2)), and finally a disordered phase (Fig. 5.2(b3)). The same trend has been observed for different particle sizes and concentrations. A well-known method to quantitatively assess the different packing structures, is to calculate the Voronoi areas [16] of the particles, as shown in Fig. 5.2(b). In Fig. 5.2(c), we identify the Voronoi areas corresponding to the different cell arrangements: the disordered phase exhibits a larger average cell area as well as a wider dispersion, compared to the ordered phase. This reveals the sharp transition from order to disorder.

\subsection{Rush hour in the drying drop}

The order-to-disorder transition can be traced back to the hydrodynamics within the drop. Fig. 5.3(a) shows the radial velocity measured by $\mu$ PIV of the layer of particles closest to the glass slide, in a region close to the contact line. The particle velocity increases dramatically in the last moments of the drop's life. We refer to this sudden change in speed as "rush hour". The particles that arrive early, at a low deposition speed, form an ordered (square or hexagonal) structure. By contrast, particles that arrive during rush hour have a high speed and form a jammed, disordered phase.

The rush-hour behavior can be explained by simple mass-balance considerations. The volume flow towards the contact line inside the drop is driven by the evaporation 


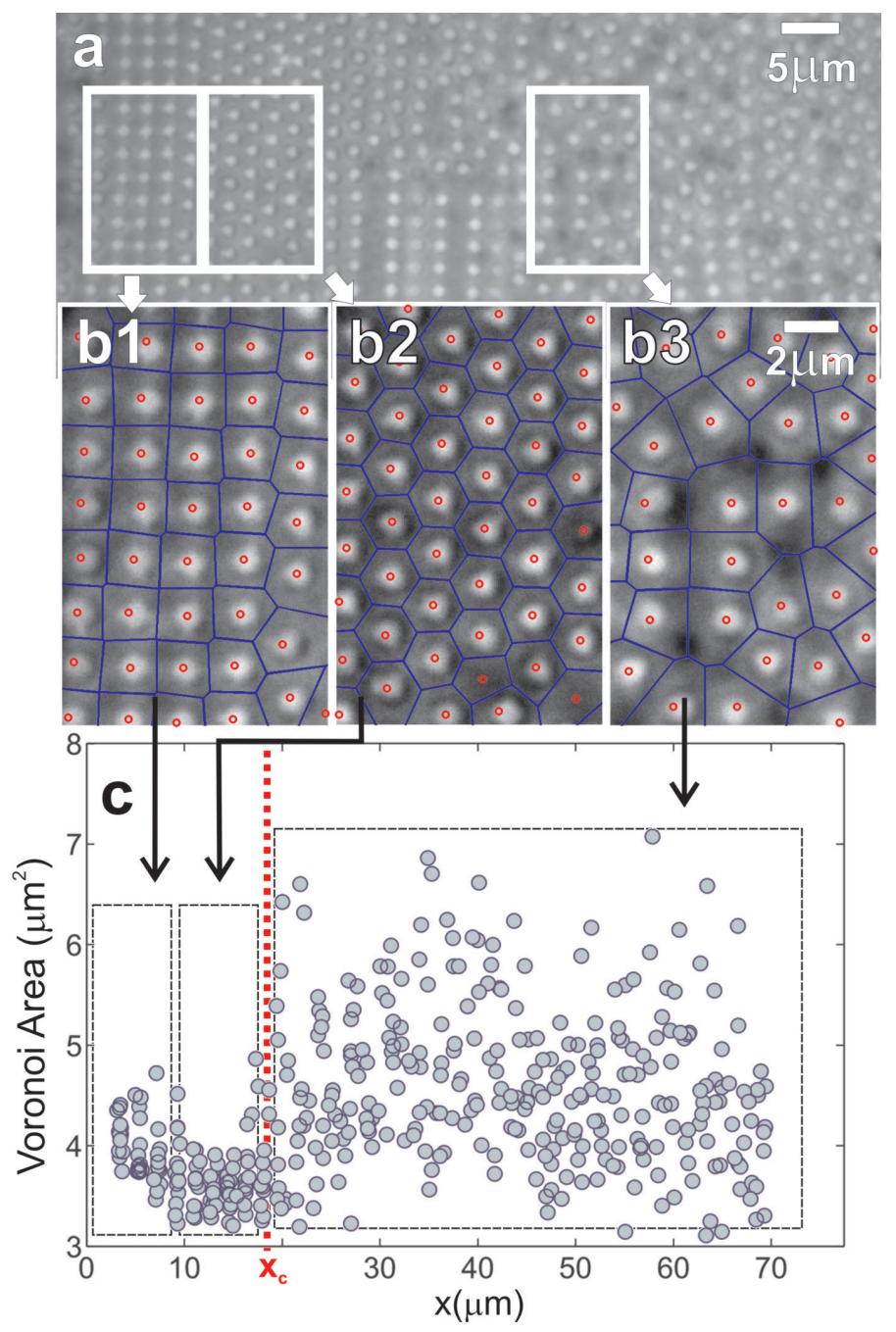

Figure 5.2: Analysis of the particle ordering in the stain. (a) Overview of the different patterns observed in the ring stain. The position of the contact line is on the left side of the figure (the first layers of particles are not shown). (b1-3) A close-up reveals the sequence of patterns in the stain: square packing close to the contact line (b1), followed by hexagonal packing (b2), and finally disordered packing (b3). The Voronoi cells belonging to the particles are shown in blue. (c) The area of the Voronoi cells plotted versus the distance $x$ from the contact line. The order-to-disorder transition at $x=x_{c}$ is determined by the increase in the mean Voronoi area and the dispersion, due to the lack of order. This transition is indicated by the red dotted line. 
from the drop surface [1]. It turns out that the rate of evaporation is approximately constant over time [6]. To replenish this evaporated liquid, a continuous volume flow towards the contact line is generated inside the drop. However, the drop height is vanishing during evaporation. This can be characterized by a contact angle $\theta(t) \rightarrow 0$ at the final stages of evaporation (see Fig. 5.3). Hence, the same amount of liquid has to be squeezed through an area which is vanishing, inducing a diverging radial velocity. Close to the contact line, the dependence of the height-averaged radial velocity $\bar{u}$ on time $t$ and distance from the drop center $r$, is given by $[4,6]$

$$
\bar{u}(r, t)=\frac{D^{*}}{\theta(t)} \frac{1}{\sqrt{R(R-r)}}
$$

see the Appendix A.2 for details. Here, $D^{*}=2 \sqrt{2} D_{v a} \Delta c /\left(\pi \rho_{\ell}\right)$ represents the driving of the flow, with $D_{v a}=24 \times 10^{-6} \mathrm{~m}^{2} / \mathrm{s}$ the diffusion constant for vapor in air, $\Delta c=$ $1.2 \times 10^{-2} \mathrm{~kg} / \mathrm{m}^{3}$ the vapor concentration difference between the drop surface and the surroundings, $\rho_{\ell}=998 \mathrm{~kg} / \mathrm{m}^{3}$ the liquid density, and $R$ the drop radius. The spatial $(R-r)^{-1 / 2}$-divergence originates from the diverging evaporative flux from the drop surface [1], as discussed in Chapter 1. Although this spatial divergence generated a lot of interest [1, 4-8], it is clear from (5.1) that there is also a temporal divergence because $\theta(t)$ vanishes. This temporal divergence provides the key to the rush-hour behavior: the radial velocity blows up towards the end of the drop's life.

The experimental velocity measurements are performed at a distance of $2 \mu \mathrm{m}$ above the glass slide, and therefore differ from the theoretical height-averaged velocity. To quantitatively compare the model with the experimental data, a description of the velocity profile is required. Given the low velocities measured inside the evaporating drop (low Reynolds number) the inertial terms in the Navier-Stokes equation can be neglected. Since the drop height is much smaller than its radius, the thin-film (lubrication) approximation [17] can be used to describe the velocity field. As discussed in Chapter 3, the lubrication approximation gives an accurate description of the velocity field inside an evaporating drop of small contact angle all the way down to the contact line. In the lubrication approximation, the velocity $u(r, z, t)$ is given by (see Appendix A.2)

$$
u(r, z, t)=\frac{3}{h^{2}(r, t)} \bar{u}(r, t)\left(h(r, t) z-\frac{1}{2} z^{2}\right)
$$

with $h(r, t)=\theta(t)\left(R^{2}-r^{2}\right) /(2 R)$, the local drop height. As shown in Fig. 5.3, this model quantitatively describes the rush-hour behaviour. For the plot of (5.2) we took $R-r=174 \mu \mathrm{m}$ (the distance from the contact line where the $\mu$-PIV measurements are performed), $z=2 \mu \mathrm{m}$ and $t_{f}$ the drop's life time determined from the experiment. 


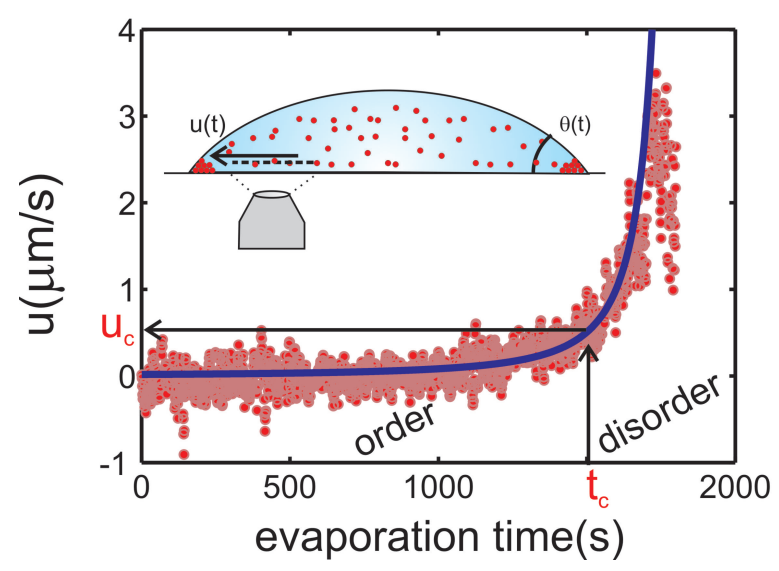

Figure 5.3: Origin of the order-to-disorder transition. Plot of the radial particle velocity versus time, measured at a distance of $174 \mu \mathrm{m}$ from the contact line. The fluid velocity predicted by the model (5.1) is plotted as a solid line. Both model and experiment show the dramatic velocity increase at the end of the drop's life (the rush hour). The inset shows the direction of the radial velocity in the drop. The time $t_{c}$ at which the order-to-disorder transition occurs is determined from the videos; the corresponding critical velocity $u_{c}$ is read from this graph (indicated by the black line in the figure).

This means there are no adjustable parameters in the comparison between the model and the experiments.

\subsection{Order-to-disorder transition}

We show that the structural change in the stain can be entirely attributed to the rushhour behaviour. The particles that form the ordered phase arrive at the contact line at an early time, when the deposition rate is still low. On the other hand, the particles that belong to the disordered phase arrive during rush hour, at a high deposition rate. The slow, early arriving particles have time to arrange themselves within the stain by Brownian motion, while those coming in rush hour have no time to find an appropriate spot and are quenched into a disordered phase. We can quantify this transition by comparing the time scale on which particles arrange by diffusion to the time scale of particle deposition. The diffusive time scale is $t_{d}=d_{p}^{2} / D$, where $D$ is the diffusivity of the particles in liquid, calculated by the Einstein relation. The hydrodynamic time 
scale is $t_{h}=L / u$ (where $L$ is the typical distance between the particles, dependent on their concentration in the solution $n_{p}(t)$ as $L=n_{p}^{-1 / 3}$, and $u$ is the particle velocity). When $t_{d}$ and $t_{h}$ become of the same order, a transition is expected. We therefore define the critical transition velocity as

$$
u_{c} \sim L D / d_{p}^{2}
$$

We validated this criterion experimentally for various particle concentrations and sizes. First, we determined the time $t_{c}$ when the ring-shaped stain undergoes the transition (the position where this occurs is $x_{c}$, as indicated by the red line in Fig. 5.2(c)). Next, we used the velocity-time plots (Fig. 5.3) to find the experimental value of $u\left(t_{c}\right)=u_{c}$. As demonstrated in Fig. 5.4, the experimental critical velocity perfectly follows the proposed scaling. The agreement suggests that the prefactor in (5.3) is actually of order unity. Our model also explains many results described in the literature [12-15], where large-scale crystalline deposits were observed for particle sizes ranging from 6 to almost $50 \mathrm{~nm}$, in hindsight. In this range of particle sizes, the critical velocity required for a disordered phase (5.3) is unreachable within the drop, which explains the absence of a disordered phase.

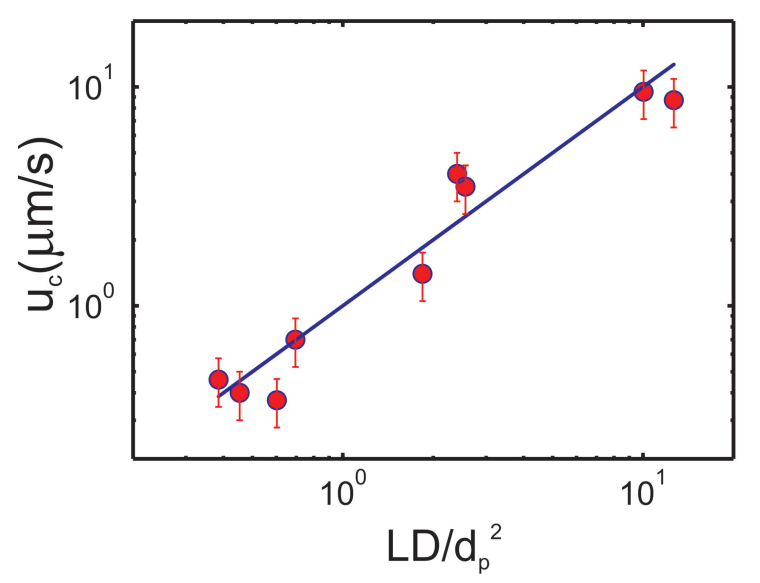

Figure 5.4: The experimentally determined critical velocity $u_{c}$, versus its theoretical prediction (5.3).

Apart from the order-to-disorder transition, there are also transitions within the ordered phase, from square to hexagonal packing and vice versa (Fig. 5.1(d)). In an unconfined system, the most efficient packing lattice is the Hexagonal Close Packing 
(HCP), as conjectured by Kepler [18]. However, in the evaporating drop the particles are confined in a wedge formed by the glass slide on one side, and the liquid-air interface with contact angle $\theta$ on the other side. It was shown by Pieranski et al. $[19,20]$ that such a confinement indeed leads to a sequence of hexagonal and square packing structures, dependent on the most efficient packing for the available space. Indeed, when a new layer is formed, the confinement by the wedge favours the square packing; see Fig 1. Away from the step edge, the available space increases and allows for the denser, hexagonal packing structure. We observe a sequence of this type of transitions, each time a new layer is added. Similar transitions were also observed by Abkarian et al. [21] in a wedge formed by a moving contact line.

\subsection{Conclusion}

In this chapter we revealed the mechanism triggering crystallization in an evaporating colloidal solution drop with a pinned contact line. The temporal divergence (rush hour) observed in the velocity field inside the drop is responsible for the lack of order in the ring-shaped stains: when the deposition rate is low, particles have time to arrange by Brownian motion and form an ordered phase. During the rush hour, there is no time for such re-arrangement and the particles form a disordered phase. Such effect resembles the popular computer game Tetris, where at the beginning of the game slowly falling objects are easily arranged in regular structures, while as the game evolves, the fast falling objects become jammed and disordered. Therefore, the key to generate large-scale colloidal crystals is to avoid the rush-hour behaviour, i.e. to avoid small contact angles during evaporation. This can be achieved for example by modifying the hydrophobicity of the substrate or by continuously replenishing the evaporated liquid. Large-scale crystalline arrays can also be obtained by tuning the particle size, since nanometre-sized particles diffuse much more efficiently. As a rule of thumb, freely-evaporating colloidal solutions with particles within the nanometric range will naturally crystallize, micrometer-sized particles will show both ordered and disordered phases, while larger particles lead to disordered stains.

\section{Appendix}

In this appendix we describe the technical details of the experimental methods and the hydrodynamic model used in this chapter. 


\section{A.1 Experimental materials and methods}

The colloids consist of monodisperse fluorescent polystyrene particles (1\% polydispersity, Thermo Scientific); various particle diameters have been tested ranging from 0.5 to $2 \mu \mathrm{m}$. The colloidal solutions were prepared with triply deionized water at concentrations ranging from $0.01 \%$ to $0.5 \% \mathrm{w} / \mathrm{w}$. No further study has been performed for solutions of different ionic strengths. A drop of this colloidal solution is gently deposited onto an untreated glass slide and left to evaporate in an empty room (no human heat sources present) with controlled temperature and humidity.

The whole evaporative process is filmed simultaneously from the side and from below (see Fig. 5.5). The particles are filmed from below with an CMOS camera (PCO imaging) connected to an inverted microscope at different magnifications, depending on the particle size, ranging from $\times 40$ for the largest, up to $\times 100$ for the smallest particles. The size of the field of view (FOV) was typically $300 \times 200 \mu \mathrm{m}^{2}$. The horizontal position of the FOV was close to the drop's contact line (about $100 \mu \mathrm{m}$ away from it) and its vertical position about one particle diameter above the glass slide. An integrated standard halogen light source is used to illuminate the particles; the emitted light is filtered before it reaches the camera. The camera is operated at a typical frame rate of 1 frame per second, which is enough to capture the movement of the particles, because the maximum velocities observed in the system were always below $100 \mu \mathrm{m} / \mathrm{s}$. A custom-made MATLAB micro-Particle Image Velocimetry $(\mu \mathrm{PIV})$ code was used to determine the velocity field in the measurement area from the captured images.

The side view images are used to calculate contact angle, volume, radius, and height of the drop at every instant. Side-view images are taken with a long distance microscope and a CCD camera (Lumenera Corp.) synchronized with the visualization of the particles by a common trigger. The side-view images are analysed with a custom-made MATLAB code, in which the detected drop profile was fit to an ellipse. The elliptical fit allowed us to use three parameters to fit the drop contour (major axis, minor axis and angle of the ellipse with the horizontal plane), leading to a higher accuracy compared to a spherical fit. The contact angle is obtained by calculating the tangent of the ellipse at the drop's contact line; the method yields errors in contact angle below $5 \%$.

A video explaining the entire process and the outcome of the rush hour can be downloaded from the winning entry of the Gallery of Fluid Motion 2010 [22]. 


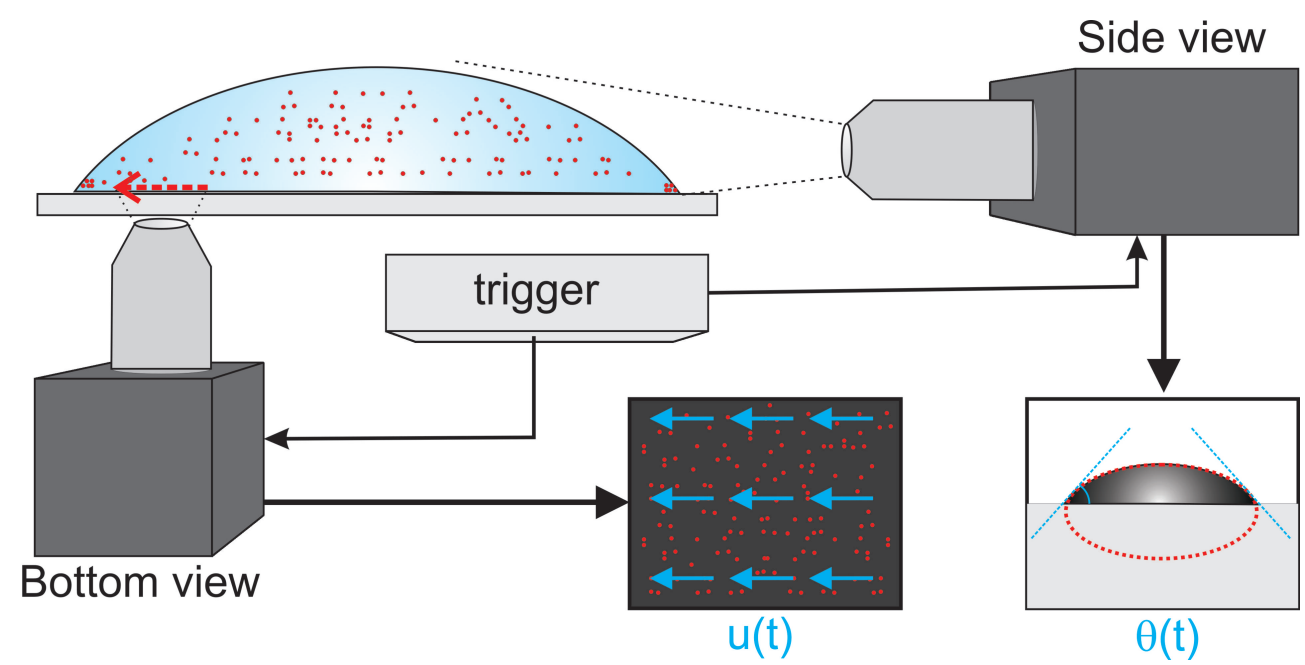

Figure 5.5: Sketch of the experimental set-up for the simultaneous side and bottom view of the evaporation process. Two synchronized cameras are used for obtaining the particle velocity and the drop's contact angle and other geometrical properties at every instant.

\section{A.2 Hydrodynamic model}

In this section, we show how the expression for the radial velocity inside an evaporating drop, (5.1), is derived from mass balance. Furthermore, we comment on the velocity profile used for the plot in Fig. 5.3.

\section{A.2.1 Mass conservation}

The volume flow inside the drop is driven by the evaporation from the drop surface. We consider an axisymmetric drop with a pinned contact line, described in a cylindrical coordinate system $(r, z)$; see Fig. 5.6. Inside this drop, we define an infinitesimally small control volume of width $d r$, located at a distance $r$ from the drop center. Mass conservation requires that the rate of change of the amount of liquid inside this control volume equals the net inflow of liquid into the control volume, minus the amount of liquid that has evaporated from the drop surface. As explained in Chapter 1, this can be expressed as [4]

$$
\frac{\partial h}{\partial t}=-\frac{1}{r} \frac{\partial}{\partial r} Q-\frac{1}{\rho_{\ell}} J,
$$




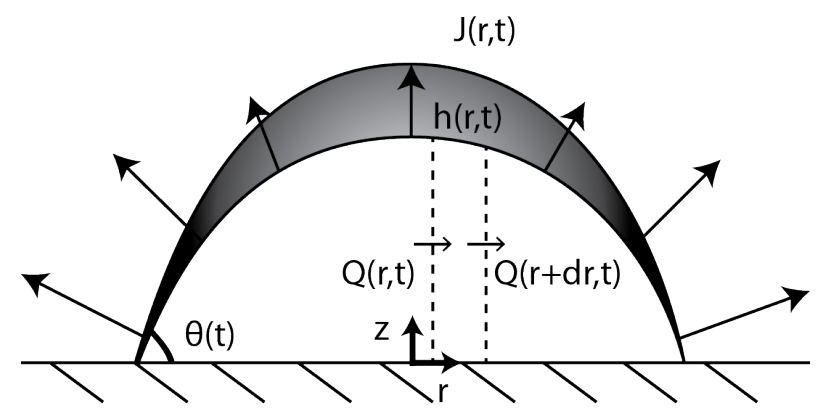

Figure 5.6: Schematic representation of an axisymmetric drop that is evaporating, described in a cylindrical coordinate system $(r, z)$. The evaporative flux $J$ from the drop surface drives a flow $Q$ inside the drop with height $h$. The dotted lines indicate the control volume of width $d r$.

where $h(r, t)$ is the local drop height, $t$ the time, $Q(r, t)$ the volume flow, $\rho_{\ell}=998$ $\mathrm{kg} / \mathrm{m}^{3}$ the liquid density, and $J(r, t)$ the evaporative flux. Our goal is to establish an expression for $Q$, from which we can deduce the velocity towards the contact line. From (5.4) we already see that if the local change in drop height is smaller than the evaporative volume flux, a flow towards the contact line is generated.

To find an expression for $h$, we consider the limit of large surface tension, such that the drop shape is not affected by the flow inside the drop, or by gravity. In this case, the drop has a spherical-cap shape. The contact angle of our water drops on glass is small: initially it is about $60^{\circ}$, while during the rush hour it is vanishing. Therefore, the drop with radius $R$ is well described by a parabola with contact angle $\theta$

$$
h(r, t)=\frac{R^{2}-r^{2}}{2 R} \theta(t) .
$$

Hence, to find $\partial h / \partial t$, we need an expression for $d \theta / d t$. Following [4-6], we assume the vapor concentration field around the drop is quasi-steady and the evaporation is diffusion dominated. The evaporative flux $J$ is then obtained from the solution of the diffusion equation for vapor in air. In the limit of small contact angles $\left(\theta \ll 90^{\circ}\right)$ this evaporative flux is constant in time, and given by

$$
J(r)=\frac{2}{\pi} \frac{D_{v a} \Delta c}{\sqrt{R^{2}-r^{2}}}
$$

with $D_{v a}=24 \times 10^{-6} \mathrm{~m}^{2} / \mathrm{s}$ the diffusion constant for vapor in air, and $\Delta c=1.2 \times 10^{-2}$ $\mathrm{kg} / \mathrm{m}^{3}$ the vapor concentration difference between the drop surface and the surround- 
ings in the experiment. To obtain an expression for $d \theta / d t$ we use global mass conservation: the change in drop volume $V_{d}$

$$
\frac{d V_{d}}{d t}=\frac{d}{d t} \int_{0}^{R} h(r, t) 2 \pi r \mathrm{~d} r=\frac{\pi R^{3}}{4} \frac{d \theta}{d t},
$$

should be equal to the total amount of evaporated liquid

$$
\frac{d V_{d}}{d t}=-\frac{1}{\rho_{\ell}} \int_{0}^{R} J(r) 2 \pi r d r=-\frac{4 R D_{v a} \Delta c}{\rho_{\ell}} .
$$

From (5.7), (5.8) it follows that

$$
\frac{d \theta}{d t}=-\frac{16 D_{v a} \Delta c}{\pi R^{2} \rho_{\ell}}
$$

which is constant over time. Hence,

$$
\theta(t)=\frac{16 D_{v a} \Delta c}{\pi R^{2} \rho_{\ell}}\left(t_{f}-t\right),
$$

with $t_{f}$ the total life time of the drop. Inserting (5.5), (5.9), and (5.6) into (5.4), we obtain by integration

$$
Q(r)=\frac{2 D_{v a} \Delta c}{\pi \rho_{\ell}}\left[\sqrt{R^{2}-r^{2}}-\frac{1}{R^{3}}\left(R^{2}-r^{2}\right)^{2}\right] .
$$

As can be observed from (5.11), the flow is constant over time. The height-averaged radial velocity $\bar{u}$ can be derived from the flow:

$$
\bar{u}(r, t)=\frac{Q}{r h}=\frac{4 R D_{v a} \Delta c}{\pi \rho_{\ell} r} \frac{1}{\theta(t)}\left[\frac{1}{\sqrt{R^{2}-r^{2}}}-\frac{1}{R^{3}}\left(R^{2}-r^{2}\right)\right] .
$$

Close to the contact line $(r \rightarrow R)$, (5.12) reduces to

$$
\bar{u}(r, t)=\frac{2 \sqrt{2} D_{v a} \Delta c}{\pi \rho_{\ell} \sqrt{R}} \frac{1}{\theta(t)} \frac{1}{\sqrt{R-r}} .
$$

In Fig. 5.7 we show the comparison of (5.13) (dark grey solid line) with our experimental data (black dots). The height-averaged model captures the temporal divergence of the velocity field, but there is no quantitative agreement with the experimental data. This is because the experimental velocity is measured close to the wall, where the actual velocity is much lower than the height-averaged velocity. 


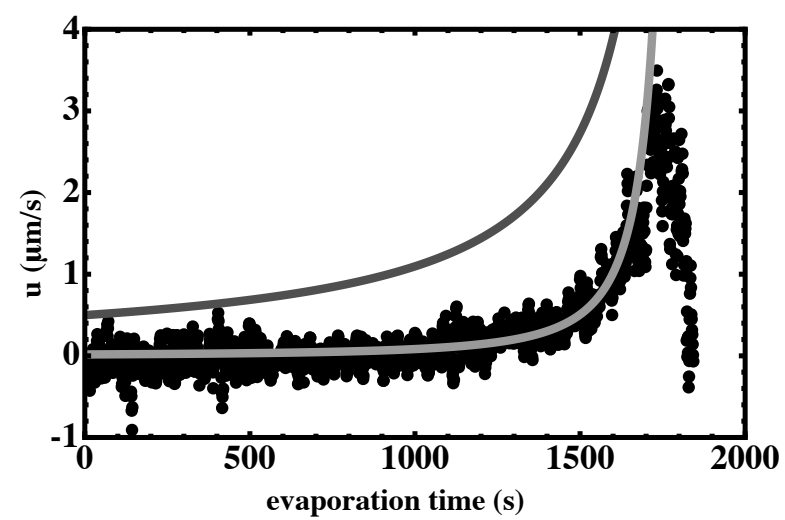

Figure 5.7: A plot of the radial velocity versus time, at a distance of $174 \mu \mathrm{m}$ from the contact line. The experimentally determined velocity field, measured at a distance of $2( \pm 0.5) \mu m$ above the glass slide, is shown as black dots, the height-averaged model (5.13) is the dark grey solid line, and the model including the velocity profile (5.17) is the light grey solid line. By taking into account the velocity profile as given by (5.17), we can quantitatively describe the experimental data without any adjustable parameters.

\section{A.2.2 Velocity profile}

The experimental velocity data depicted in Fig. 5.7 are measured at a distance of 2 $\mu \mathrm{m}$ above the glass slide, and therefore differs from the theoretical height-averaged velocity. To quantitatively compare the model with the experimental data, a description of the velocity profile is required. Given the low velocities measured inside the evaporating drop (low Reynolds number) the inertial terms in the Navier-Stokes equation can be neglected. Since the drop height is much smaller than its radius, the thin-film (lubrication) approximation [17] can be used to describe the velocity field, and Navier-Stokes reduces to

$$
\frac{d p}{d r}=\mu \frac{\partial^{2} u}{\partial z^{2}}
$$

with $p$ the pressure, $\mu$ the dynamic viscosity, and $u$ the radial velocity. According to (5.14), the velocity profile is given by

$$
u(r, z)=\frac{1}{\mu} \frac{d p}{d r}\left(\frac{1}{2} z^{2}-h(r, t) z\right)
$$


where we used the boundary conditions $u(r, 0)=0$ (no slip) and $\left.\frac{\partial u}{\partial z}\right|_{z=h(r, t)}=0$ (no shear stress on the liquid-air interface). Taking the height average of (5.15), we obtain

$$
\bar{u}(r, t)=-\frac{1}{3 \mu} \frac{d p}{d r} h^{2}(r, t),
$$

hence

$$
u(r, z, t)=\frac{3}{h^{2}(r, t)} \bar{u}(r, t)\left(h(r, t) z-\frac{1}{2} z^{2}\right),
$$

with $h$ given by (5.5), $\bar{u}$ by (5.12), and $\theta$ by (5.10). Fig. 5.7 shows that when the velocity profile (5.17) is taken into account, we can quantitatively describe the experimental data (light grey solid line). For the plot of (5.17) we took $R-r=174 \mu \mathrm{m}$, $z=2 \mu \mathrm{m}$ and $t_{f}$ the drop's life time determined from the experiment. This means there are no adjustable parameters left.

\section{References}

[1] R. D. Deegan, O. Bakajin, T. F. Dupont, G. Huber, S. R. Nagel, and T. A. Witten, "Capillary flow as the cause of ring stains from dried liquid drops", Nature 389, 827-828 (1997).

[2] A. Stein and R. Schroden, "Colloidal crystal templating of three-dimensionally ordered macroporous solids: materials for photonics and beyond", Curr. Opin. Solid St. M. 5, 553-564 (2001).

[3] L. Zhang, S. Maheshwari, H. Chang, and Y. Zhu, "Evaporative self-assembly from complex dna-colloid suspensions", Langmuir 24, 3911-3917 (2008).

[4] R. D. Deegan, O. Bakajin, T. F. Dupont, G. Huber, S. R. Nagel, and T. A. Witten, "Contact line deposits in an evaporating drop", Phys. Rev. E 62, 756765 (2000).

[5] H. Hu and R. G. Larson, "Analysis of the microfluid flow in an evaporating sessile droplet”, Langmuir 21, 3963-3971 (2005).

[6] Y. O. Popov, "Evaporative deposition patterns: Spatial dimensions of the deposit”, Phys. Rev. E 71, 036313 (2005).

[7] H. Bodiguel and J. Leng, "Imaging the drying of a colloidal suspension", Soft Matter 6, 5451-5460 (2010). 
[8] H. Eral, D. Augustine, M. Duits, and F. Mugele, "Suppressing the coffee stain effect: how to control colloidal self-assembly in evaporating drops using electrowetting", Soft Matter 7, 4954-4958 (2011).

[9] K. P. Velikov, C. G. Christova, R. P. A. Dullens, and A. van Blaaderen, "Layerby-layer growth of binary colloidal crystals", Science 296, 106 -109 (2002).

[10] D. J. Harris, H. Hu, J. Conrad, and J. Lewis, "Patterning colloidal films via evaporative lithography", Phys. Rev. Lett. 98, 148301 (2007).

[11] F. Fan and K. J. Stebe, "Assembly of colloidal particles by evaporation on surfaces with patterned hydrophobicity", Langmuir 20, 3062-3067 (2004).

[12] T. P. Bigioni, X. M. Lin, T. T. Nguyen, E. I. Corwin, T. A. Witten, and H. M. Jaeger, "Kinetically driven self assembly of highly ordered nanoparticle monolayers", Nat. Mater. 5, 265-270 (2006).

[13] L. Pauchard, F. Parisse, and C. Allain, "Influence of salt content on crack patterns formed through colloidal suspension desiccation", Phys. Rev. E 59, 3737 (1999).

[14] C. Allain and L. Limat, "Regular patterns of cracks formed by directional drying of a collodial suspension”, Phys. Rev. Lett. 74, 2981-2984 (1995).

[15] E. Dufresne, E. Corwin, N. A. Greenblatt, J. Ashmore, D. Wang, A. Dinsmore, J. Cheng, X. Xie, J. Hutchinson, and D. Weitz, "Flow and fracture in drying nanoparticle suspensions", Phys. Rev. Lett. 91, 224501 (2003).

[16] G. Voronoi, "Nouvelles applications des paramètres continus à la théorie des formes quadratiques", Journal für die Reine und Angewandte Mathematik 133, 97-178 (1907).

[17] A. Oron, S. H. Davis, and S. G. Bankoff, "Long-scale evolution of thin liquid films”, Rev. Mod. Phys. 69, 931-980 (1997).

[18] J. Kepler, Strena seu de nive sexangula, 1611 (English translation, Clarendon Press, Oxford) (1966).

[19] P. Pieranski, L. Strzelecki, and B. Pansu, "Thin colloidal crystals", Phys. Rev. Lett. 50, 900-903 (1983).

[20] B. Pansu, P. Pieranski, and P. Pieranski, "Structures of thin layers of hard spheres: high pressure limit”, J. Physique 45, 331-339 (1984). 
[21] M. Abkarian, J. Nunes, and H. A. Stone, "Colloidal crystallization and banding in a cylindrical geometry”, J. Am. Chem. Soc. 126, 5978-5979 (2004).

[22] A. G. Marín, H. Gelderblom, J. H. Snoeijer, and D. Lohse, "Avalanche of particles in evaporating coffee drops", Winning entry Gallery of Fluid Motion APS-DFD 2010, available online at http://pof.aip.org/gallery_of_fluid_motion2011_galleryrushhour_in_evaporating_coffee_drops (2010). 


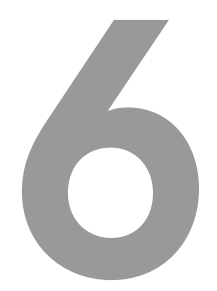

\section{Building microscopic soccer balls with evaporating colloidal fakir drops * $\dagger$}

In the previous chapter we have shown that the evaporation of colloidal dispersion drops with a pinned contact line can lead to the formation of ordered two-dimensional structures. Evaporation-driven particle self-assembly can also be used to generate three-dimensional microstructures. In this chapter, we present a new method to create these colloidal microstructures, in which we can control the amount of particles and their packing fraction. To this end, we let colloidal dispersion drops evaporate from a special type of superhydrophobic microstructured surface, on which the drop remains in Cassie-Baxter state during the entire evaporative process. The remainders of the drop consist of a massive spherical cluster of the microspheres, with diameters ranging from a few tens up to several hundreds of microns. We present scaling arguments to show how the final particle packing fraction of these balls depends on the dynamics of the drop evaporation, particle size, and number of particles in the system.

*Published as:

Álvaro G. Marín, Hanneke Gelderblom, Arturo Susarrey-Arce, Arie van Houselt, Leon Lefferts, Han Gardeniers, Detlef Lohse, and Jacco H. Snoeijer, "Building microscopic soccer balls with evaporating colloidal fakir drops”, Proc. Natl. Acad. Sci. U.S.A. 109 (41), 16455-16458 (2012).

${ }^{\dagger}$ The experimental work is performed by Álvaro G. Marín and Arturo Susarrey-Arce. 


\subsection{Introduction}

Evaporation-driven particle self-assembly is an ideal mechanism for constructing micro- and nanostructures at scales where direct manipulation is impossible. For example, in colloidal dispersion drops with pinned contact lines, as discussed in the previous chapter, evaporation gives rise to the so-called coffee-stain effect [1]: a capillary flow drags the particles towards the contact line to form a ring-shaped stain. Such a flow not only aggregates the particles, but is also able to organize them in crystalline phases [2-5]. Similar mechanisms such as the "convective assembly" $[6,7]$ are currently successfully used to produce two-dimensional colloidal crystal films. To obtain three-dimensional clusters of micro-particles, colloidal dispersion drops can be dryed suspended in emulsions [8-10], in spray dryers [11, 12] or kept in Leidenfrost levitation [13]. The main drawback of these three-dimensional assembly techniques, however, is the lack of control on both the amount of particles and the particle arrangement in the remaining structures.

In this work, we devise a unique, controlled way of generating on-demand selfassembled spherical micro-structures via drop evaporation on a superhydrophobic surface (see Fig. 6.1). We present scaling arguments to predict the particle arrangement in the microstructures formed, based on the dynamics of the evaporation process.

\subsection{Colloidal supraballs}

To generate the microstructures, we evaporate colloidal dispersion drops from a special type of superhydrophobic substrates. In most of the cases, a liquid Cassie-Baxter state drop evaporating from a superhydrophobic surface will eventually suffer a wetting transition into a Wenzel state, i.e. it will get impaled into the structure and loose its spherical shape $[14,15]$. Here, however, we use a surface that combines overhanging pillared structures $[16,17]$ with a hierarchical nano-structure (Fig. 6.3(c)). These surface properties impose a huge energy barrier for the wetting transition to occur, and therefore the drop will remain almost floating over the structure in a CassieBaxter state during its entire life [18].

A typical result can be observed in Fig. 6.1: a water drop containing $1 \mu \mathrm{m}$ soluble polystyrene particles (initial concentration $0.08 \%$ weight and initial volume $5 \mu \mathrm{l}$ ) evaporates on the superhydrophobic surface at room temperature and $30 \%$ humidity. After a typical evaporation time of 45 minutes, the solvent is completely evaporated and only the colloids are left upon the substrate. Remarkably, the particles $\left(\sim 10^{7}\right.$ in this particular case) are not just lying scattered on the pillars but they have aggregated 


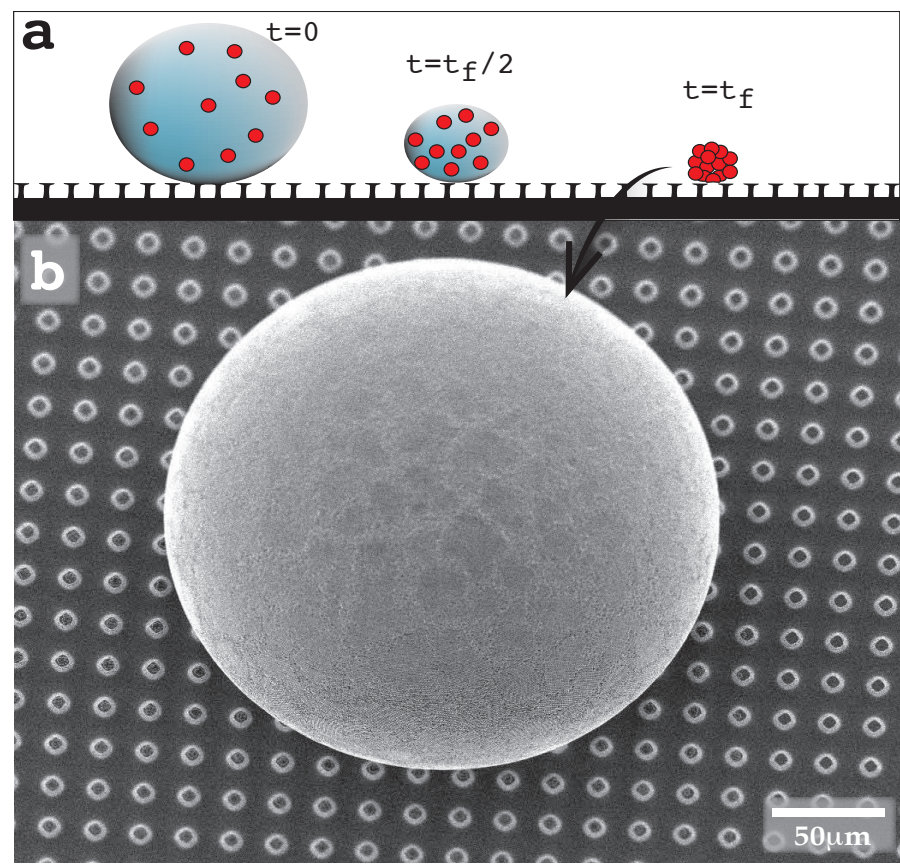

Figure 6.1: (a) A drop of colloidal solution is left to evaporate on a superhydrophobic surface. As the solvent evaporates, the particle concentration increases. Once all the solvent has completely evaporated the colloidal particles have aggregated to form a spherical particle conglomerate: a colloidal supraball. (b) Top view of the resultant compact colloidal supraball left on the superhydrophobic surface after evaporation. The micropillars forming the structure are seen as circular objects around the supraball.

and form a spherical macro-cluster resting on top of the micro-pillars, which we call colloidal supraball. The final ball has a slightly oblate shape, with an ellipticity of approximately $10 \%$. The final macroscopic contact angle with the structure is slightly (about 15\%) lower than the initial one. Since the particles are fully wetted and do not have the tendency to accumulate at the interface, spontaneous shell formation [10] is not expected. Indeed, we do not observe shell formation during the evaporation of the drops [11, 13, 19]: the supraballs we obtain are solid, and present a high mechanical resistance and stability. In order to inspect inside the ball, we cut some of them open with the aid of sharp blades or micro-capillaries. All the examined balls showed compact nuclei and no hollow structures (see Fig. 6.2). Additionally, 

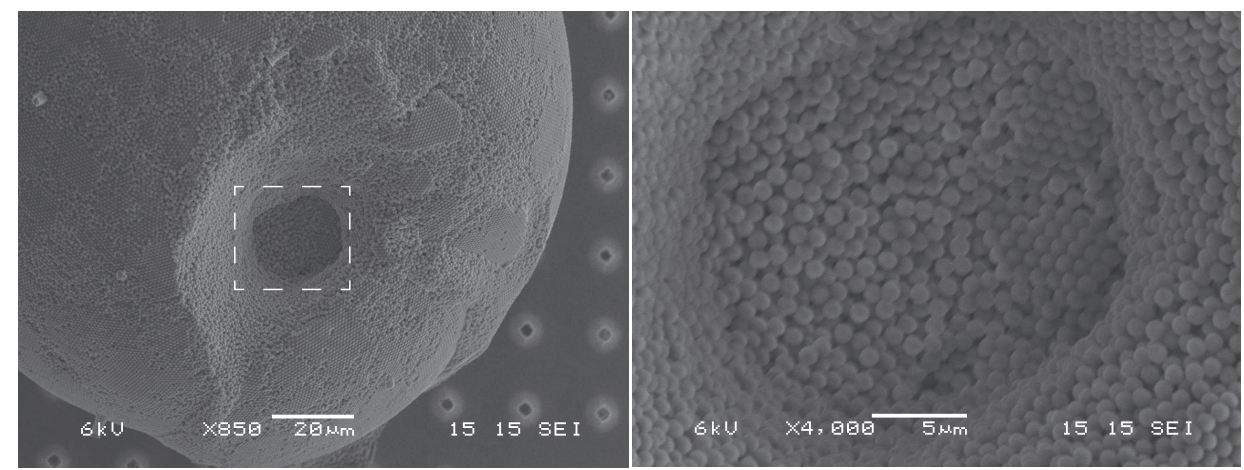

Figure 6.2: A perforated supraball: one of the most compact balls was perforated using a microcapillary with a tip of $20 \mu \mathrm{m}$. The observation confirms that the supraballs have a relatively high compacity even close to its core.

when looking closer at the surface of these particular colloidal supraballs as shown in Fig. 6.3(d), one can identify crystalline flat patches which resemble the pentagonal patches present in a soccer ball. These crystalline patches are even observed close to the contact line (see Fig. 6.3(b)), and at the bottom of the ball, where its shape is slightly flattened due to the contact with the pillars.

The system strongly differs from the particle deposition in the coffee-stain problem $[1,5]$, where pinning of the drop's contact line causes a capillary-driven flow that brings the particles to the boundary. Pauchard and Couder [20] studied the drying of highly-concentrated colloidal solutions of large drops on hydrophobic surfaces, which is more similar to our system. However, in their case the contact line is also pinned, and as a consequence the drop does not keep its spherical shape but shows strong deformations.

\subsection{Drop evaporation}

To understand the final structure of the supraballs, it will turn out crucial to understand the dynamics of the drop evaporation. The fact that we do not observe shell formation, suggests that the particles do not influence the drop evaporation. To test this hypothesis, we compare the evaporation dynamics to that of a liquid drop that does not contain any particles. The evaporative mass loss from such a drop is typically governed by the diffusion of vapour molecules in the surrounding air [1,21, 22]. 


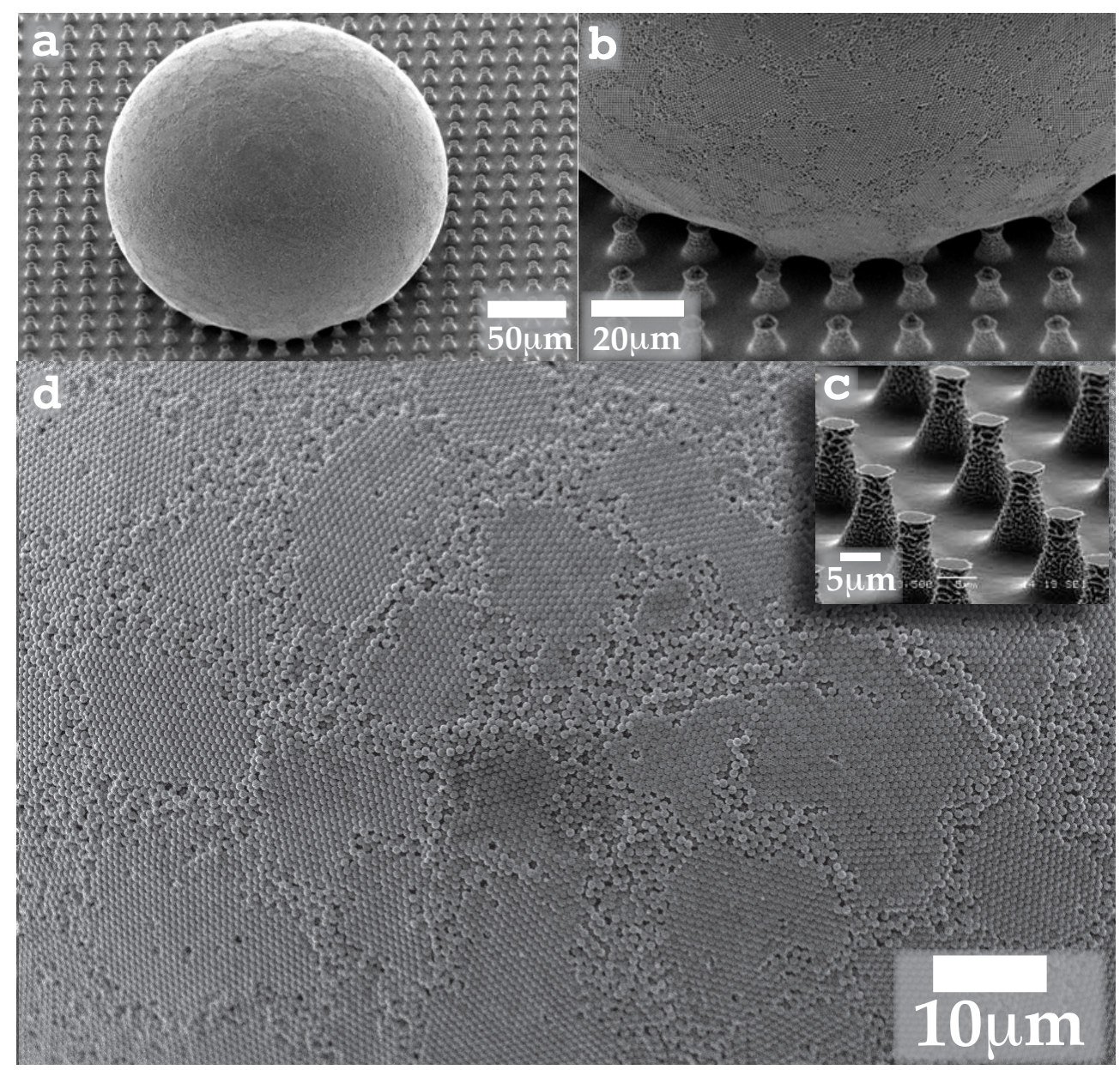

Figure 6.3: (a) Tilted view of the supraball in contact with the microstructure. (b) Detail of the contact area. (c) Magnified view of the micropillars forming the microstructure. (d) Close-up of the supraball surface. The distribution of crystalline patches resemble the pentagons in a soccer ball. 
For diffusion-limited evaporation, the rate of volume change of the drop is given by (see Chapter 1)

$$
\frac{d V}{d t} \sim D^{\prime} R
$$

where $R$ is the drop radius, and $D^{\prime}=D_{v a} \Delta c / \rho_{\ell}$, with $D_{v a}$ the diffusion constant for vapour in air, $\Delta c$ the vapour concentration difference between drop surface and the surroundings and $\rho_{\ell}$ the liquid density [5]. One might have expected the evaporation rate from the drop surface to be proportional to the drop surface area $\sim R^{2}$. However, the vapour concentration gradient is proportional to $1 / R$, and therefore the total evaporation rate is proportional to $R$ [23]. If the drop evaporates with a constant contact angle, we find that, since $V \sim R^{3}$,

$$
R(t) \sim\left[D^{\prime}\left(t_{f}-t\right)\right]^{1 / 2}
$$

Here $t_{f}$ is the total drop lifetime in case no particles are present, for which the drop radius reaches zero. In the present case the drop radius saturates at a finite radius, $R_{\text {ball }}$, at a time $\hat{t}=t_{f}-R_{b a l l}^{2} / D^{\prime}$, corresponding to the moment where the particles become densely packed. In Fig. 6.4 we plot the colloidal drop radius versus $t_{f}-t$. The drop radius is measured using a spherical/elliptical fitting from side-view images of the shrinking drop during the experiment. Our experimental data for different number of particles are in very good agreement with the 1/2-power law. This result confirms that the particles do not influence the evaporation process, until the final radius $R_{\text {ball }}$ is reached. Hence, we see no indication that the particles form an impermeable shell during the evaporation of the drop. In previous studies, Sen et al. [11, 19] concluded that a shell would only form for fast evaporation or in extremely dilute cases. The absence of a shell also agrees with other well-known colloidal drop evaporation experiments in which particles do not tend to adsorb at the interface [1,5], unless the colloids are specifically modified for that purpose [10,24].

The scaling (6.1) implies that the speed with which the interface is moving inwards, is given by $d R / d t \sim D^{\prime} / R$. Hence, the interface speed increases dramatically as the drop shrinks and the maximum speed reached in the experiment will be determined by the final radius $R_{\text {ball }}$. As we will show further on, this increase in interface speed determines the particle packing inside the supraballs.

\subsection{Packing fraction of the supraballs}

The final size of the ball depends on the number of particles inside the drop. This can be tuned by manipulating either the initial particle concentration or the drop size. In our experiment, the ball size was in the range $100<R_{\text {ball }} / R_{p}<1000$, with $R_{p}$ the 


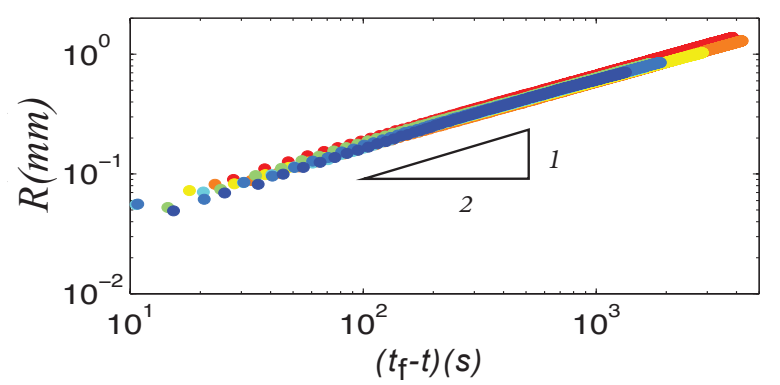

Figure 6.4: The radius of the drop plotted against $t_{f}-t$ with $t_{f}$ the lifetime of the drop and $t$ the actual time. The triangle indicates the 1/2-power law, the dots represent the data sets for 7 different experiments, where the number of particles was varied. For a certain $\hat{t}<t_{f}$ the final ball size $R_{\text {ball }}$ is reached. The final time was extrapolated as $t_{f}=\hat{t}+R_{b a l l}^{2} / D^{\prime}$.

particle radius. Clearly, the exact final size of the ball will not only depend on the amount of particles in the system but also on their packing fraction. We define the global packing fraction as

$$
\Phi \equiv N\left(\frac{R_{p}}{R_{\text {ball }}}\right)^{3}
$$

where $N$ is the total number of particles in the drop. The final supraball radius $R_{\text {ball }}$ is accurately determined from Scanning Electron Microscope (SEM) images. If the packing fractions were identical for all supraballs, one would expect that $R_{\text {ball }} / R_{p} \sim$ $N^{1 / 3}$. In Fig. 6.5(a) however, we show that there is some deviation from the $1 / 3$ scaling, especially for balls with a smaller number of particles ${ }^{\dagger}$. In Fig. 6.5(b) we show that the packing fraction indeed strongly depends on the number of particles in the system.

As the number of particles increases, the packing fraction approaches that of a perfect Hexagonal Close Packing configuration, in which case one would find $\Phi=0.74$ [25], hence, we have an ordered particle packing inside the balls. On the other hand, the supraballs with a smaller amount of particles show remarkably low packing fractions, even below the Random Close Packing $(\mathrm{RCP})$ limit $(\Phi=0.64)$

\footnotetext{
$\dagger$ All quantitative results shown in this chapter have been performed with colloids of $1 \mu \mathrm{m}$ diameter (non-surface-modified fluorescent microspheres supplied by Thermo Scientific), but the same qualitative behavior has been observed for 0.2 and $2 \mu \mathrm{m}$. The colloidal solutions were always prepared with deionized water. According to the manufacturers, the particles have a melting point of $\sim 200^{\circ} \mathrm{C}$.
} 

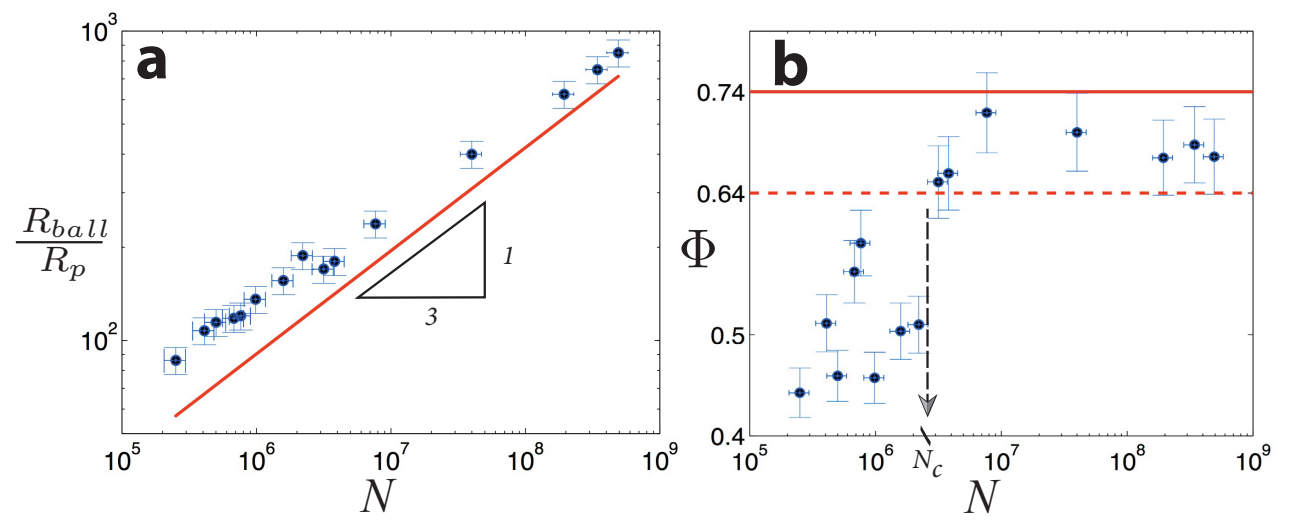

Figure 6.5: (a) Supraball to microparticle diameter $R_{\text {ball }} / R_{p}$ plotted against the total amount of particles $N$ in the system. (b) The packing fraction $\Phi$ strongly depends on $N$. The dots represent experimental measurements and the solid line corresponds the most efficient particle packing $\Phi=0.74$ (hexagonal close packed), the dashed line marks $\Phi=0.64$, random close packed. $N_{c}$ is the critical number of particles, above which we find an ordered ball structure.

[26], corresponding to a disordered particle arrangement. The balls which show packing fractions below the RCP limit contain several empty cavities. Remarkably, the final configuration reached, seems to depend on the number of particles in the system. In Fig. 6.5(b) we indicated the critical number of particles $N_{c} \approx 3 \cdot 10^{6}$ when the packing fraction reaches that of a RCP. For $N<N_{c}$ we get a loose, disordered particle packing in the supraball, whereas for $N>N_{c}$ we get a densely packed, ordered supraball.

\subsection{Order-to-disorder transition}

What causes the transition from ordered to disordered packings, and what determines the critical number of particles? In order to answer these questions, we follow a similar approach as in the previous chapter: we compare the time scale on which particles can arrange by diffusion to the hydrodynamic time scale for the particle transport by convection, given by the inward motion of the liquid-air interface. If the diffusion time is small compared to the hydrodynamic time, particles can arrange into an ordered packing. The diffusive time scale for particles to find the best position in a crystalline phase is given by $t_{d}=R_{p}^{2} / D$, with $R_{p}$ the particle radius and $D$ the 
diffusivity of the particles in the liquid $[3,5,27]$. The hydrodynamic time-scale is $t_{h}=L /\left|\frac{d R}{d t}\right|$. Here $R(t)$ is the drop radius, and $L$ is the typical inter-particle distance, which depends on the particle concentration as $L=N^{-1 / 3} R$, as long as the solution is dilute $\left(L \gg R_{p}\right)$. We define the ratio of both time scales as:

$$
\mathscr{A}(t) \equiv \frac{t_{d}}{t_{h}}=\left|\frac{d R(t)}{d t}\right| \frac{t_{d}}{L}=\frac{D^{\prime}}{D} N^{1 / 3}\left(\frac{R_{p}}{R(t)}\right)^{2},
$$

where in the last step we used (6.1) to replace $d R / d t \sim D^{\prime} / R$. Note that our definition of the interparticle distance $L$ is a global average of the whole system. Locally, some inhomogeneities will be present, in particular close to the interface, but our scaling analysis should not be affected.

From (6.4) we observe that $\mathscr{A}(t)$ increases as the drop radius becomes smaller during the evaporation (see Fig. 6.4), until the limit $R=R_{\text {ball }}$ is reached. A cross-over between the time-scales is reached when the hydrodynamic time becomes equal to the diffusion time, hence when $\mathscr{A}=1$. If the cross-over is reached when $R \gg R_{\text {ball }}$, the amount of crystalline clusters is still very small. From this point in time onwards the interface speed is too high for the particles to further arrange in a crystalline way [5]. Instead, they are pushed together in a random arrangement, with a low packing fraction. If the cross-over is reached when $R \leq R_{\text {ball }}$, the particle packing is already dense and ordered, and we find a high packing fraction. For all drops the evaporative mass loss, and hence the decrease in radius, is the same (see Fig. 6.4), hence, the moment when the particle packing becomes sufficiently dense for particles to arrange depends solely on the number of particles in the drop. If $N$ is high $\left(N>N_{c}\right)$, this moment is reached relatively early, i.e. well before $\mathscr{A}=1$, and we get an ordered particle packing inside the supraballs. Using that $R_{\text {ball }} / R_{p} \sim N^{1 / 3}$ and considering $\mathscr{A}=1$, we find from (6.4) the critical number of particles above which we obtain ordered supraballs

$$
N_{c} \sim\left(\frac{D^{\prime}}{D}\right)^{3}
$$

This result emphasizes that the transition is governed by two diffusion processes: the diffusion of vapour, determining the speed of evaporation, versus the diffusion of particles inside the drop. The ratio of diffusion constants selects the critical number of particles. In our experiment $D^{\prime}=3 \times 10^{-10} \mathrm{~m}^{2} / \mathrm{s}$ and $D=2 \times 10^{-13} \mathrm{~m}^{2} / \mathrm{s}$, from which we find that $N_{c} \sim 10^{9}$. This prediction is two to three orders of magnitudes larger than the experimentally observed $N_{c}$. There are two reasons why this estimation is quantitatively off: first of all, we have neglected all prefactors, and the result is strongly (to the third power) dependent on the experimental parameters included 
in $D^{\prime}$, i.e. humidity, liquid density, diffusivity of vapour, and saturated vapour concentration. Secondly, in the analysis we have not taken into account which packing fraction is actually achieved when $\mathscr{A}=1$.

To verify whether the final packing fraction indeed depends on the spacing between the particles the moment the cross-over time is reached, we go back to our experimental data. We define the time-dependent packing fraction as $N\left(R_{p} / R(t)\right)^{3}$. As the drop evaporates, this packing fraction will increase until it reaches its final value $\Phi$. At the cross-over, defined by $\mathscr{A}=1$, the drops will have a packing fraction $\Phi^{*}$. If this $\Phi^{*}$ is low, the amount of crystalline clusters is still very small. On the other hand, if $\Phi^{*}$ is high, we expect crystalline clusters to have formed already. After the cross-over time, the interface moves too fast to allow for further ordering, and it just presses the ordered particle clusters closer together. In Fig. 6.6 we show that drops with a high $\Phi^{*}$ have a high $\Phi$ : when $\Phi^{*} \gtrsim 0.1$ we obtain a final packing fraction above the RCP limit.

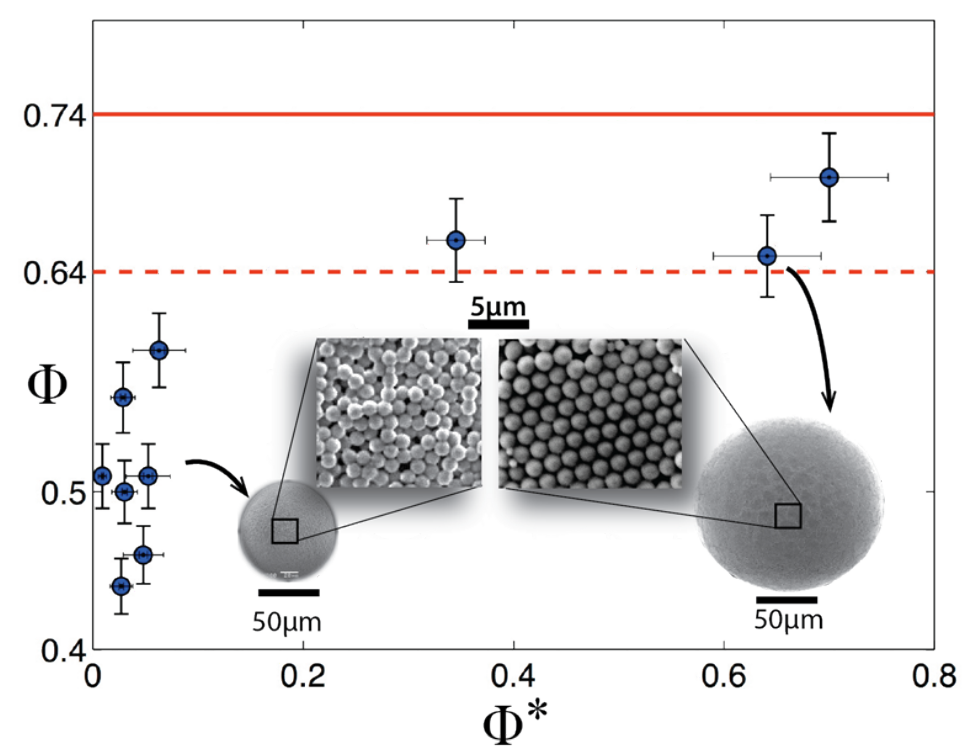

Figure 6.6: Final packing fraction $\Phi_{f}$ versus the packing fraction at the cross-over time $\Phi^{*}$. Drops below a certain $\Phi^{*}$ have a too low packing fraction at the cross-over time to achieve final packing fractions above the RCP limit. The particle packing can not only be obtained from the value of the packing fraction, but it can also directly be observed from the SEM images of the surface of the supraballs. 
We cannot predict the critical $\Phi^{*}$ theoretically. However, we can, retrospectively, use the experimental critical $\Phi^{*}$ to compute $N_{c}$. Using that $R_{\text {ball }} / R_{p} \sim 0.1 N^{1 / 3}$ at the cross-over, we obtain $N_{c} \sim 10^{7}$, which is in the same order of magnitude as our experimental results; see Fig. 6.5(b).

The particle packing in the supraballs can not only be assessed by measuring the packing fraction, but it can also directly be seen in SEM images from the surface of the colloidal supraballs, as shown in Fig. 6.6. The size of the soccer-ball-like crystalline patches on the surface of the ordered supraballs depends on the ball size: bigger balls will show larger patches due to the reduced curvature at their surfaces. To explain the size of the crystalline domains, we hypothesize that a crystalline patch will bend radially no more than a particle size. Then, it follows by simple trigonometry that the size of a patch $S$ will be related to the ball radius $R_{\text {ball }}$ and the particle size $R_{p}$ via: $S / R_{\text {ball }}=\arccos \left(1-R_{p} / R_{\text {ball }}\right)$. This expression predicts a typical patch size of $\sim 7 \mu \mathrm{m}$ for a ball with $R_{\text {ball }}=50 \mu \mathrm{m}$ and $R_{p}=0.5 \mu \mathrm{m}$, which is in the right order of magnitude as one can observe in Fig. 6.3(d).

\subsection{Conclusion}

In this chapter we described a simple technique to create spherical colloidal supraballs relying only on drop evaporation from a robust superhydrophobic surface. The supraballs show a highly ordered structure if the number of particles inside the drop is large enough to trigger early particle clustering. The critical number of particles required to obtain an ordered particle packing inside the balls depends on the parameters driving the drop evaporation (through $D^{\prime}$ ) and the diffusivity of the particles. Hence, by controlling the humidity and ambient temperature the supraball packing fraction and hence size can be controlled. Massive fabrication of micro-compactsupraballs could easily be achieved by simply spraying a colloidal solution over the micro-structure in a controlled atmosphere. By tuning the wetting properties of the particles one could also be able to generate the well-known colloidosomes [10] using the same proposed technique.

\section{References}

[1] R. D. Deegan, O. Bakajin, T. F. Dupont, G. Huber, S. R. Nagel, and T. A. Witten, "Capillary flow as the cause of ring stains from dried liquid drops", Nature 389, 827-828 (1997). 
[2] F. Fan and K. J. Stebe, "Assembly of colloidal particles by evaporation on surfaces with patterned hydrophobicity", Langmuir 20, 3062-3067 (2004).

[3] T. P. Bigioni, X. M. Lin, T. T. Nguyen, E. I. Corwin, T. A. Witten, and H. M. Jaeger, "Kinetically driven self assembly of highly ordered nanoparticle monolayers", Nat. Mater. 5, 265-270 (2006).

[4] D. J. Harris, H. Hu, J. Conrad, and J. Lewis, "Patterning colloidal films via evaporative lithography", Phys. Rev. Lett. 98, 148301 (2007).

[5] A. G. Marín, H. Gelderblom, D. Lohse, and J. H. Snoeijer, "Order-to-disorder transition in ring-shaped colloidal stains", Phys. Rev. Lett. 107, 085502 (2011).

[6] K. P. Velikov, C. G. Christova, R. P. A. Dullens, and A. van Blaaderen, "Layerby-layer growth of binary colloidal crystals", Science 296, 106 -109 (2002).

[7] L. Meng, H. Wei, A. Nagel, J. Benjamin, L. Scriven, and D. Norris, "The role of thickness transitions in convective assembly", Nano Letters 6, 2249-2253 (2006).

[8] O. D. Velev, A. M. Lenhoff, and E. W. Kaler, "A class of microstructured particles through colloidal crystallization”, Science 287 (2000).

[9] V. Manoharan, M. Elsesser, and D. Pine, "Dense packing and symmetry in small clusters of microspheres", Science 301, 483 (2003).

[10] A. Dinsmore, M. Hsu, M. Nikolaides, M. Marquez, A. Bausch, and D. Weitz, "Colloidosomes: selectively permeable capsules composed of colloidal particles”, Science 298, 1006 (2002).

[11] D. Sen, S. Mazumder, J. Melo, A. Khan, S. Bhattyacharya, and S. D'Souza, "Evaporation driven self-assembly of a colloidal dispersion during spray drying: volume fraction dependent morphological transition", Langmuir 25, 6690-6695 (2009).

[12] D. Sen, O. Spalla, O. Taché, P. Haltebourg, and A. Thill, "Slow drying of a spray of nanoparticles dispersion. in situ saxs investigation", Langmuir 23, 42964302 (2007).

[13] N. Tsapis, E. Dufresne, S. Sinha, C. Riera, J. Hutchinson, L. Mahadevan, and D. Weitz, "Onset of buckling in drying droplets of colloidal suspensions", Phys. Rev. Lett. 94 (2005). 
[14] M. Reyssat, J. Yeomans, and D. Quéré, "Impalement of fakir drops", Europhys. Lett. 81, 26006 (2008).

[15] P. Tsai, R. G. H. Lammertink, M. Wessling, and D. Lohse, "Evaporationtriggered wetting transition for water droplets upon hydrophobic microstructures", Phys. Rev. Lett. 104, 116102 (2010).

[16] A. Tuteja, W. Choi, J. Mabry, G. McKinley, and R. Cohen, "Robust omniphobic surfaces”, Proc. Natl. Acad. Sci. 105, 18200 (2008).

[17] R. Dufour, P. Brunet, M. Harnois, R. Boukherroub, V. Thomy, and V. Senez, "Zipping effect on omniphobic surfaces for controlled deposition of minute amounts of fluid or colloids", Small (2012).

[18] A. Susarrey-Arce, A. G. Marín, H. Nair, L. Lefferts, J. Gardeniers, D. Lohse, and A. van Houselt, "Absence of an evaporating-driven wetting transition on omniphobic surfaces”, Soft Matter xx (2012).

[19] D. Sen, J. Melo, J. Bahadur, S. Mazumder, S. Bhattacharya, G. Ghosh, D. Dutta, and S. D'Souza, "Buckling-driven morphological transformation of droplets of a mixed colloidal suspension during evaporation-induced self-assembly by spray drying”, Eur. Phys. J. E 31, 393-402 (2010).

[20] L. Pauchard and Y. Couder, "Invagination during the collapse of an inhomogeneous spheroidal shell”, Europhys. Lett. 66, 667 (2004).

[21] Y. O. Popov, "Evaporative deposition patterns: Spatial dimensions of the deposit”, Phys. Rev. E 71, 036313 (2005).

[22] H. Gelderblom, A. G. Marín, H. Nair, A. van Housselt, L. Lefferts, J. H. Snoeijer, and D. Lohse, "How water droplets evaporate on a superhydrophic substrate”, Phys. Rev. E 83, 026306 (2011).

[23] J. Eggers and L. M. Pismen, "Nonlocal description of evaporating drops", Phys. Fluids 22, 112101 (2010).

[24] P. J. Yunker, T. Still, M. A. Lohr, and A. G. Yodh, "Suppression of the coffeering effect by shape-dependent capillary interactions", Nature 476, 308-311 (2011).

[25] J. Kepler, Strena seu de nive sexangula, 1611 (English translation, Clarendon Press, Oxford) (1966). 
[26] D. A. Weitz, "Packing in the spheres", Science 303, 968-969 (2004).

[27] K. E. Davis, W. Russel, and W. Glantschnig, "Disorder-to-order transition in settling suspensions of colloidal silica- x-ray measurements", Science $\mathbf{2 4 5}$, 507-510 (1989) 


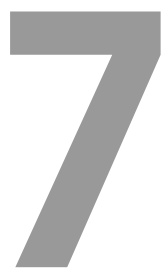

\section{Oscillations of a gas pocket on a liquid-covered solid surface *}

The dynamic response of a gas bubble entrapped in a cavity on the surface of a submerged solid subject to an acoustic field is investigated in the linear approximation. We derive semi-analytical expressions for the resonance frequency, damping and interface shape of the bubble. For the liquid phase, we consider two limit cases: potential flow and unsteady Stokes flow. The oscillation frequency and interface shape are found to depend on two dimensionless parameters: the ratio of the gas stiffness to the surface tension stiffness, and the Ohnesorge number, representing the relative importance of viscous forces. We perform a parametric study and show, among others, that an increase in the gas pressure or a decrease in the surface tension leads to an increase in the resonance frequency until an asymptotic value is reached.

\subsection{Introduction}

The volume pulsations of a gas pocket entrapped on a liquid-covered solid surface constitute a fundamental problem at the root of several applications in biology, microfluidics, cavitation and others. For example, the oscillatory flow induced by the

${ }^{*}$ Published as: Hanneke Gelderblom, Aaldert G. Zijlstra, Leen van Wijngaarden, and Andrea Prosperetti, "Oscillations of a gas pocket on a liquid-covered solid surface", Phys. Fluids 24, 122101 (2012). 
pulsations causes a liquid motion which can be used to study the behaviour of bacteria and cells under the action of shear [see e.g. 1-3]. In these conditions sonoporation of cell walls may occur, which would facilitate the uptake of drugs [see e.g. 4] and gene transfection [see e.g. 5]. The flow induced by the oscillating gas pocket also induces mixing and streaming [see e.g. 6]. Under large-amplitude acoustic excitation, small gas bubbles issue from the gas entrapped in the cavities which greatly enhance sonochemical reactions in a more controlled way than is possible in a conventional sonoreactor [7]. Microfabricated cavities on a silicon surface have been used to study controlled cavitation and bubble growth and collapse [8,9].

Despite this wide range of applications, little is known about the dynamic response of a gas pocket on a submerged solid in an acoustic field. Miller [10] and Neppiras et al. [11] recorded the acoustic response of multiple bubbles entrapped in a membrane. However, their size was not controlled, and no information about the response of the individual bubbles could be obtained. Rathgen et al. [12] studied the dynamics of periodic arrays of gas-filled micropores of controlled size on a solid surface. Using optical diffraction techniques, they were able to resolve in time, with a high accuracy, the nanometre-scale oscillations of the gas-liquid menisci driven by a sound field. However, they were unable to resolve the shape of the menisci in the course of the oscillations.

Theoretical studies mainly focused on spherical bubbles in the bulk liquid [13] whereas for crevice bubbles only approximate results exist. Miller \& Nyborg [14] derived approximate expressions for the lowest resonance frequency and damping of a gas-filled pore on a solid surface under the assumption that the interface shape is parabolic. Their result is that the lowest resonance frequency $f_{0}$ of a cylindrical pore with radius $a$ and depth $h$ is approximately given by

$$
f_{0}=\frac{1}{2 \pi a} \sqrt{\frac{15 \pi \kappa \lambda p_{0} a+120 \pi \sigma}{32 \rho_{\ell} a}},
$$

with $\kappa$ the polytropic index, $\lambda=a / h$ the aspect ratio of the pore, $p_{0}$ the gas pressure when the interface is flat, $\sigma$ the surface tension coefficient, and $\rho_{\ell}$ the liquid density. As an example, upon taking $\lambda=1$, this relation predicts natural frequencies of 176 , 17.6 , and $1.76 \mathrm{kHz}$ for gas pockets of air in water with equivalent spherical radii of 10, 100, and $1000 \mu \mathrm{m}$. To obtain (7.1), an energy argument was used. Rathgen et al. [12] improved somewhat on this estimate by formulating the correct hydrodynamic problem for the liquid phase. However, they only solved the problem in an approximate way, retaining the parabolic approximation for the free-surface shape and also considering only the lowest resonance frequency.

The purpose of the present work is to study the dynamics of the liquid-gas in- 
terface bounding the gas contained in a cavity at the surface of a solid in the linear approximation. We calculate the frequency, damping and surface shape of the linear normal modes of oscillation of the system in the inviscid and viscous cases. In many situations the resonance frequency of the system is mainly determined by the inertia of the liquid, and hence can be calculated with sufficient accuracy from a potential flow model. We estimate the damping in two ways: from the potential flow solution by using a dissipation function method and, more accurately, by solving the time-dependent Stokes equations. The dynamics of the liquid-gas interface is found to depend on two dimensionless parameters: the ratio of the gas stiffness to the surface tension stiffness, and the Ohnesorge number, representing the viscous damping during one period of oscillation.

\subsection{Problem formulation}

Our aim is to describe the resonance frequency and interface shape of a gas bubble entrapped in a crevice. We model the crevice as a cavity with a circular mouth at the surface of an infinite solid submerged in an incompressible liquid (Fig. 7.1). The cavity has an aspect ratio

$$
\lambda=\pi a^{3} / V_{0},
$$

with $a$ the mouth radius and $V_{0}$ the cavity volume when the interface is flat. For a cylindrical cavity, $\lambda=a / h$ with $h$ the depth of the cavity, but the results that follow hold for cavities of arbitrary shape. We introduce a cylindrical coordinate system $(r, z)$, with the origin located on the axis of the cavity mouth at the level of the infinite solid plane. The liquid-gas interface is assumed to remain pinned at the circular edge of the cavity. The elevation of the free surface over the plane $z=0$ is described by $\eta(r, t)$, and is assumed to be small compared to the radius of the cavity mouth, $\eta<<a$.

When the interface is perturbed, the compression of the gas and the surface tension of the liquid-air interface provide restoring forces and the interface will start to oscillate around its equilibrium position, which is assumed to be flat. The oscillating interface causes a velocity field $\boldsymbol{u}$ and pressure field $p$ in the liquid phase (the gas flow is neglected here). To calculate the oscillation frequency, damping and shape of the interface we need to couple the normal stress in the liquid, derived from the velocity field, to the pressure in the gas, which results from the effect of surface tension and the gas compression and expansion.

We use a linear theory, in which the time-dependence is assumed to be proportional to $e^{\zeta t}$, so that $\eta(r, t)=\eta(r) e^{\zeta t}$, with complex eigenfrequency $\zeta=i \omega-\beta$, where $\omega=2 \pi f$ is the angular frequency and $\beta$ the damping coefficient due to viscous 


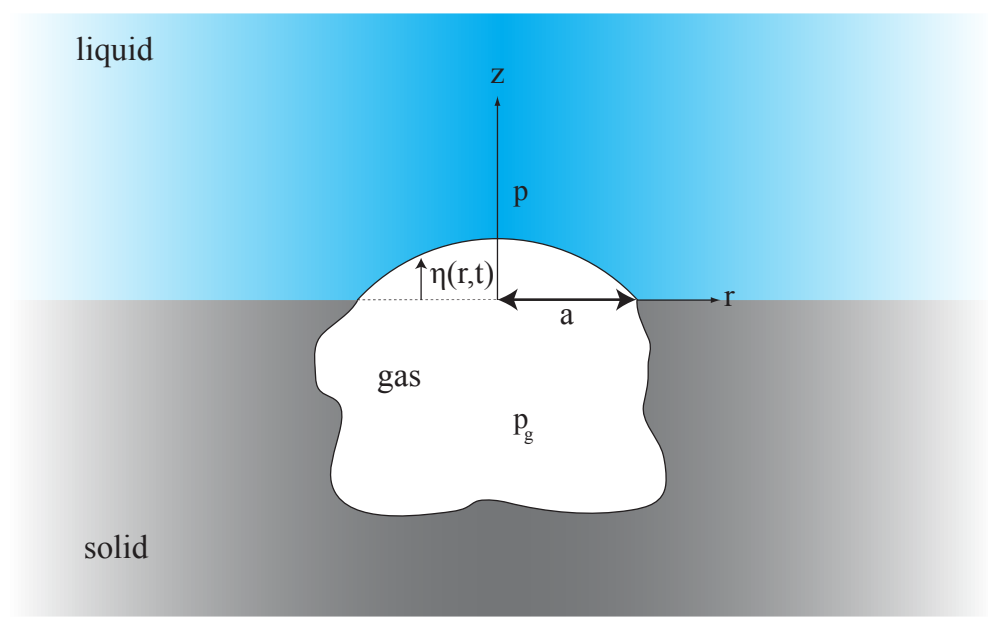

Figure 7.1: A cavity with an entrapped gas bubble. The radius of the circular cavity mouth $a$ is indicated, as well as the cylindrical coordinate system $(r, z)$. The pressures in the gas and in the liquid are $p_{g}$ and $p$, respectively. The elevation of the free surface above the $(z=0)$-plane is denoted by $\eta(r, t)$.

dissipation in the liquid. This time-dependence is left implicit in the expressions that follow. The motion of the liquid-air interface, described by $S(r, t)=z-\eta(r, t)=0$, is coupled to the velocity field in the liquid via the kinematic condition

$$
\frac{\partial S}{\partial t}+\boldsymbol{u} \cdot \nabla S=0 .
$$

Since we consider a small interface deformation $\eta$, we neglect all terms which are of second order and higher, which leads to a kinematic boundary condition in the form

$$
\left.u_{z}\right|_{z=0}=\zeta \eta, \quad 0 \leq r / a<1 .
$$

The solid is impermeable for the liquid, and therefore we impose along the remainder of the $(z=0)$-plane

$$
\left.u_{z}\right|_{z=0}=0, \quad 1<r / a<\infty .
$$

Furthermore, as there is no slip on the solid surface,

$$
\left.u_{r}\right|_{z=0}=0, \quad 1<r / a<\infty .
$$


On a clean liquid-gas interface, a no-shear-stress boundary condition applies, since the gas viscosity is much smaller than the liquid viscosity:

$$
\tau_{r z}=\mu\left(\frac{\partial u_{r}}{\partial z}+\frac{\partial u_{z}}{\partial r}\right)=0, \quad 0 \leq r / a<1,
$$

with $\mu$ the dynamic viscosity. The presence of impurities modifies the interfacial behaviour and may be modelled by a no-slip condition [15]

$$
\left.u_{r}\right|_{z=0}=0, \quad 0 \leq r / a<1 .
$$

Since the mixed boundary value problem (7.6), (7.7) leads to a mathematical problem which does not appear to be solvable, we impose the no-slip conditions (7.6), (7.8) on the entire surface in the following analysis.

The coupling of the pressures in the liquid and the gas occurs via the dynamic boundary condition at $z=0$

$$
p_{g}=p+\sigma \mathscr{C}-\left.2 \mu \frac{\partial u_{z}}{\partial z}\right|_{z=0},
$$

with $p_{g}$ the pressure in the gas bubble and $\mathscr{C}=-\left(\partial_{r}^{2} \eta+\partial_{r} \eta / r\right)$ the curvature of the free surface; the last term on the right-hand side is the viscous normal stress on the interface. A general relation between the gas volume $V$ and the gas pressure is given by the polytropic expression

$$
\frac{p_{g}}{p_{0}}=\left(\frac{V_{0}}{V}\right)^{\kappa}
$$

with $\kappa$ the polytropic index; $\kappa=1$ is applicable to isothermal conditions. When the interface is flat $(\eta=0), V=V_{0}$ and $p_{g}=p_{0}$, the ambient pressure in the liquid. By expanding (7.10) for small interface deformations, we find

$$
p_{g} \approx p_{0}\left[1-\kappa\left(\frac{V}{V_{0}}-1\right)\right] .
$$

The gas volume can be found from the interface shape by integration:

$$
V=V_{0}+2 \pi \int_{0}^{a} \eta r \mathrm{~d} r=V_{0}\left[1+\lambda \frac{H}{a^{3}}\right]
$$

with

$$
H=2 \int_{0}^{a} r \eta(r) \mathrm{d} r
$$


proportional to the volume change of the gas due to the interface deformation. Combining (7.11) and (7.12), we obtain

$$
p_{g}=p_{0}\left(1-\kappa \lambda \frac{H}{a^{3}}\right)
$$

and the dynamic boundary condition (7.9) becomes

$$
p_{0}\left(1-\kappa \lambda \frac{H}{a^{3}}\right)=p-\sigma\left(\partial_{r}^{2} \eta+\frac{1}{r} \partial_{r} \eta\right)-\left.2 \mu \frac{\partial u_{z}}{\partial z}\right|_{z=0} .
$$

In the following sections, the pressure in the liquid will be calculated in two limit cases: potential flow on one hand, as described in Section 7.3, and unsteady Stokes flow on the other, in Section 7.5. In addition, an estimate of the viscous damping is obtained using a modified potential flow model, where we calculate the dissipation in the bulk from the potential-flow solution (Section 7.4). Before we proceed, we introduce the following dimensionless quantities

$$
\hat{r}=\frac{r}{a}, \hat{z}=\frac{z}{a}, \hat{\eta}=\frac{\eta}{a}, \hat{u}=\sqrt{\frac{a \rho_{\ell}}{\sigma}} u, \hat{\zeta}=\sqrt{\frac{a^{3} \rho_{\ell}}{\sigma}} \zeta, \hat{p}=\frac{a}{\sigma} p, \hat{H}=\frac{H}{a^{3}}
$$

which we will use from now on, thereby dropping the carets.

\subsection{Potential flow}

To calculate the liquid pressure used in the dynamic boundary condition (7.15) we first neglect the influence of viscosity completely. Then, the flow is irrotational. As the resonance frequency of the system is mainly determined by the inertia in the liquid, it can be obtained from an inviscid flow model with a fair accuracy, as will be seen later. In the framework of potential flow theory, the no-slip condition (7.6) at the solid substrate in combination with either (7.7) or (7.8) cannot be enforced.

\subsubsection{Governing equations}

For irrotational flow, the velocity field can be written in terms of the potential $\phi$ as $\boldsymbol{u}=\nabla \phi$, which satisfies the Laplace equation $\nabla^{2} \phi=0$. At the liquid-gas interface $\phi$ has to satisfy the kinematic boundary condition (7.4)

$$
\left.u_{z}\right|_{z=0}=\left.\partial_{z} \phi\right|_{z=0}=\zeta \eta, \quad 0 \leq r<1,
$$


and at the solid substrate the impermeability condition (7.5)

$$
\left.u_{z}\right|_{z=0}=\left.\partial_{z} \phi\right|_{z=0}=0, \quad 1<r<\infty .
$$

We express the (dimensionless) pressure in the liquid using the linearised Bernoulli integral

$$
p=\frac{a p_{0}}{\sigma}-\zeta \phi
$$

and, neglecting the viscous stress, find for the dynamic boundary condition at the gas-liquid interface (7.15)

$$
P H-\partial_{r}^{2} \eta-\frac{1}{r} \partial_{r} \eta=\left.\zeta \phi\right|_{z=0}, \quad 0 \leq r<1,
$$

with

$$
P=\frac{a \kappa \lambda p_{0}}{\sigma}
$$

the ratio of the gas stiffness to the surface tension stiffness.

\subsubsection{Reduction to an eigenvalue problem}

As shown in Appendix A.1, the Hankel transform can be used to reduce (7.17)-(7.20) to a set of dual integral equations in terms of $\phi$. The solution of this system results in the following expression for the dynamic boundary condition (7.20)

$$
P H-\partial_{r}^{2} \eta-\frac{1}{r} \partial_{r} \eta=-\zeta^{2} \int_{0}^{\infty} v(k) J_{0}(k r) \mathrm{d} k
$$

with

$$
v(k)=\int_{0}^{1} \eta(s) s J_{0}(k s) \mathrm{d} s .
$$

Integration of (7.22) leads to the following eigenvalue problem to be solved for $\zeta$ and $\eta$

$$
\eta(r)=\frac{1}{4} P H\left(r^{2}-1\right)+\zeta^{2} \int_{0}^{\infty} \frac{v(k)}{k^{2}}\left[J_{0}(k)-J_{0}(k r)\right] \mathrm{d} k .
$$

To find the solution of this integral equation we expand the interface deformation $\eta$ into a Fourier-Bessel series

$$
\eta(r, t)=\sum_{k=1}^{\infty} c_{k}(t) J_{0}\left(j_{k} r\right)
$$


with $j_{k}$ denoting the $k$ th zero of the Bessel function $J_{0}$. Substituting (7.25) into (7.24) and taking the inner product with $r J_{0}\left(j_{n} r\right)$, we obtain the following generalized eigenvalue problem for the eigenfrequency $\zeta$ (see Appendix A.1 for details)

$$
2 P \sum_{k=1}^{\infty} \frac{J_{1}\left(j_{k}\right)}{j_{k}} \frac{J_{1}\left(j_{n}\right)}{j_{n}} c_{k}+\frac{1}{2} c_{n} j_{n}^{2} J_{1}^{2}\left(j_{n}\right)=-\zeta^{2} \sum_{k=1}^{\infty} c_{k} J_{1}\left(j_{k}\right) J_{1}\left(j_{n}\right) j_{k} j_{n} f\left(j_{k}, j_{n}\right),
$$

with $f\left(j_{k}, j_{n}\right)$ given by (7.56).

\subsection{Weak viscous effects}

In a real flow, viscous dissipation in both the bulk of the liquid and the boundary layer on the solid surface dampen the bubble oscillations. The ratio of dissipation in the boundary layer to dissipation in the bulk is given by $a / \delta$ [16], where $\delta \sim \sqrt{v / \omega}$ is the viscous boundary layer thickness. If we scale the angular frequency on the basis of the free bubble Minnaert frequency, $\omega \sim\left(\kappa p_{0} / \rho_{\ell}\right)^{1 / 2} / a$, we find that the ratio of damping in the boundary layer to damping in the bulk is given by

$$
\frac{\kappa p_{0} a^{2}}{\rho_{\ell} v^{2}}=\frac{P}{\lambda \mathrm{Oh}^{2}},
$$

with Oh the Ohnesorge number, defined by

$$
\mathrm{Oh}=\sqrt{\frac{\rho_{\ell} v^{2}}{\sigma a}} .
$$

This dimensionless parameter is a measure of the damping during one period of oscillation. In case the bulk dissipation dominates, i.e. for smaller pits, we can use the potential flow solution to estimate the damping coefficient [16].

To describe the oscillations of the damped system, we use a Lagrangian formulation complemented by the Rayleigh dissipation function. We again express the interface shape in terms of the Fourier-Bessel series (7.25). The motion of the system is now given by

$$
\frac{\partial}{\partial t}\left(\frac{\partial \mathscr{L}}{\partial \dot{c}_{k}}\right)-\frac{\partial \mathscr{L}}{\partial c_{k}}=-\mathrm{Oh} \frac{\partial \mathscr{R}}{\partial \dot{c}_{k}}
$$

with $\dot{c}_{k}=\zeta c_{k}, \mathscr{L}=\mathscr{E}_{k}-\mathscr{E}_{p}$ the Lagrangian, $\mathscr{R}$ the Rayleigh dissipation function, defined as $\mathscr{R}=\mathscr{D} / 2$ with $\mathscr{D}$ the rate of viscous dissipation in the liquid [17]. To find an expression for $\mathscr{L}$ in terms of $c_{k}$, we calculate the kinetic energy $\mathscr{E}_{k}$ and potential energy $\mathscr{E}_{p}$ of the system. The dimensionless kinetic energy of the liquid can be expressed as [18]

$$
\mathscr{E}_{k}=\frac{1}{2} \int_{V} u_{i}^{2} \mathrm{~d} V=\frac{1}{2} \int_{A} \phi \frac{\partial \phi}{\partial x_{i}} n_{i} \mathrm{~d} A,
$$


where $\boldsymbol{n}$ represents the unit surface normal directed out of the liquid. Due to the impermeability condition (7.18), the integral (7.30) reduces to an integral over the bubble interface:

$$
\mathscr{E}_{k}=-\pi \int_{0}^{1} \phi(r, 0) \partial_{t} \eta r \mathrm{~d} r
$$

The potential energy of the system can increase by an increase in area through the effect of surface tension or by a decrease in volume through compression of the gas

$$
\mathscr{E}_{p}=\int_{A_{0}}^{A} \mathrm{~d} A-\int_{V_{0}}^{V}\left(p_{g}-p_{0}\right) \mathrm{d} V=\pi \int_{0}^{1}\left(\partial_{r} \eta\right)^{2} r \mathrm{~d} r+\frac{1}{2} \pi P H^{2},
$$

with $P$ given by (7.21). The rate of viscous dissipation in potential flow reads [18]

$$
\mathscr{D}=\frac{1}{2} \mathrm{Oh} \int_{V}\left(\frac{\partial u_{i}}{\partial x_{k}}+\frac{\partial u_{k}}{\partial x_{i}}\right)^{2} \mathrm{~d} V=2 \mathrm{Oh} \int_{A} u_{i} \frac{\partial u_{i}}{\partial x_{k}} n_{k} \mathrm{~d} A .
$$

By integrating (7.33) over the surface we arrive at

$$
\mathscr{D}=-\left.8 \pi \mathrm{Oh} \int_{0}^{1} \frac{\partial \phi}{\partial r}\right|_{z=0} \partial_{r} \eta r \mathrm{~d} r .
$$

Substituting (7.25) into (7.31), (7.32), and (7.34), we can express the kinetic energy, potential energy, and dissipation in terms of the degrees of freedom $c_{k}$ and $\dot{c}_{k}$ as

$$
\begin{aligned}
& \mathscr{E}_{k}=\pi \sum_{k=1}^{\infty} \sum_{l=1}^{\infty} \dot{c}_{k} \dot{c}_{n} j_{k} j_{n} J_{1}\left(j_{k}\right) J_{1}\left(j_{n}\right) f\left(j_{k}, j_{n}\right), \\
& \mathscr{E}_{p}=\frac{1}{2} \pi \sum_{k=1}^{\infty} j_{k}^{2} c_{k}^{2} J_{k}^{2}\left(j_{k}\right)+2 \pi P \sum_{k=1}^{\infty} \sum_{l=1}^{\infty} \frac{c_{k}}{j_{k}} \frac{c_{l}}{j_{l}} J_{1}\left(j_{k}\right) J_{1}\left(j_{l}\right), \\
& \mathscr{D}=8 \pi \mathrm{Oh} \sum_{k=1}^{\infty} \sum_{l=1}^{\infty} \dot{c}_{k} \dot{c}_{n} j_{k} j_{n} J_{1}\left(j_{k}\right) J_{1}\left(j_{n}\right) g\left(j_{k}, j_{n}\right) .
\end{aligned}
$$

with $f\left(j_{k}, j_{n}\right)$ given by (7.56), and $g\left(j_{k}, j_{n}\right)$ given by (7.71). Substituting (7.35)(7.37) into (7.29) and replacing $\dot{c}_{k}$ by $\zeta c_{k}$, we obtain

$$
\begin{aligned}
2 P \sum_{k=1}^{\infty} \frac{J_{1}\left(j_{k}\right)}{j_{k}} \frac{J_{1}\left(j_{n}\right)}{j_{n}} c_{k}+\frac{1}{2} c_{n} j_{n}^{2} J_{1}^{2}\left(j_{n}\right) & =-\zeta^{2} \sum_{k=1}^{\infty} c_{k} J_{1}\left(j_{k}\right) J_{1}\left(j_{n}\right) j_{k} j_{n} f\left(j_{k}, j_{n}\right) \\
& -4 \zeta \mathrm{Oh} \sum_{k=1}^{\infty} c_{k} J_{1}\left(j_{n}\right) J_{1}\left(j_{k}\right) j_{n} j_{k} g\left(j_{n}, j_{k}\right)
\end{aligned}
$$

Note that putting Oh to zero in (7.38), i.e. neglecting the viscous dissipation, leads to exactly the same equation as derived before for potential flow, which reconfirms (7.26). 


\subsection{Unsteady Stokes flow}

In the previous sections potential flow was used to calculate the pressure and velocities in the liquid. On the solid substrate, however, the no-slip boundary condition (7.6) applies. Hence, a viscous boundary layer develops on the substrate, which cannot be accounted for in a potential flow model. Therefore, we repeat the calculation of the liquid pressure using an unsteady Stokes flow model. For small interface deformations $\eta \ll a$, as is the case here, the nonlinear term of the Navier Stokes equations can be neglected with respect to the unsteady inertia term, and the unsteady Stokes equations describe the flow in the entire domain [16].

\subsubsection{Governing equations}

The unsteady Stokes equations in dimensionless form, with the dimensionless quantities as defined in (7.16), read

$$
\zeta \boldsymbol{u}=-\nabla p+\mathrm{Oh} \nabla^{2} \boldsymbol{u}
$$

with Oh the Ohnesorge number defined in (7.28). We express the velocity in terms of a stream function $\Psi$ as

$$
\boldsymbol{u}=\nabla \times\left(\frac{1}{r} \Psi \boldsymbol{e}_{\theta}\right)=-\frac{\partial}{\partial z}\left(\frac{1}{r} \Psi\right) \boldsymbol{e}_{r}+\frac{1}{r} \frac{\partial \Psi}{\partial r} \boldsymbol{e}_{z} .
$$

Taking the curl of (7.39), we obtain

$$
\zeta \mathbf{\Omega}=\mathrm{Oh} \nabla^{2} \boldsymbol{\Omega}
$$

with vorticity $\boldsymbol{\Omega}=\nabla \times \boldsymbol{u}=-\nabla^{2}\left(\Psi / r \boldsymbol{e}_{\theta}\right)$. As boundary conditions we have again the kinematic condition (7.4) and impermeability of the substrate (7.5). As mentioned before, we impose a no-slip condition on the solid (7.6) as well as on the bubble surface (7.8), to render the mathematical problem tractable. The dynamic boundary condition now reads

$$
P H-\partial_{r}^{2} \eta-\frac{1}{r} \partial_{r} \eta=\left.p\right|_{z=0}, \quad 0 \leq r<1,
$$

with the pressure to be calculated from (7.39). Note that the normal viscous stress drops out from (7.42) as a consequence of (7.8) which, from the equation of continuity, implies that $\partial u_{z} / \partial z=0$ on $z=0$. 


\subsubsection{Reduction to an eigenvalue problem}

The solution to (7.41) can again be expressed in terms of the Hankel transform. Then, the eigenvalue problem to be solved for $\zeta, \eta$ is given by (details of the calculation can be found in Appendix A.2)

$$
\begin{aligned}
\eta(r) & =\frac{1}{4} P H\left(r^{2}-1\right) \\
& -\int_{0}^{\infty} \frac{v(k)}{k^{2}}\left[J_{0}(k)-J_{0}(k r)\right]\left[\zeta^{2}+\zeta \mathrm{Oh}\left(k^{2}+k \sqrt{k^{2}+\zeta / \mathrm{Oh}}\right)\right] \mathrm{d} k, \quad 0 \leq r<1 .
\end{aligned}
$$

Again, we expand the interface deformation $\eta$ into the Fourier-Bessel series (7.25), and obtain (see Appendix A.1 for details)

$$
\begin{aligned}
& 2 P \sum_{k=1}^{\infty} \frac{c_{k}}{j_{k} j_{n}} J_{1}\left(j_{k}\right) J_{1}\left(j_{n}\right)+\frac{1}{2} c_{n} j_{n}^{2} J_{1}^{2}\left(j_{n}\right)= \\
& \sum_{k=1}^{\infty} c_{k} j_{k} j_{n} J_{1}\left(j_{k}\right) J_{1}\left(j_{n}\right) \int_{0}^{\infty} \frac{J_{0}^{2}(s)}{\left(j_{k}^{2}-s^{2}\right)\left(j_{n}^{2}-s^{2}\right)}\left(\zeta^{2}+\zeta \mathrm{Oh}\left[s^{2}+s \sqrt{s^{2}+\zeta / \mathrm{Oh}}\right]\right) \mathrm{d} s .
\end{aligned}
$$

\subsection{Numerical solution method}

To find the resonance frequency and interface shape in the potential flow model, the generalized eigenvalue problem (7.26) is truncated to $K$ terms and solved numerically with Mathematica 8 (Wolfram Research) for $\zeta$ and $c_{k}$; see Appendix A.1. We studied the convergence of the sum (7.25) for the first three modes by taking up to $K=100$ terms into account for $P=0$ to $P=500$. We found that the system converges rapidly: for the lowest mode $K=3$ was already sufficient for accurate reconstruction of the interface shape. For higher modes, the matrix size increases because more Bessel functions are required to describe the interface shape: for mode 3, we used $K=6$. The larger the matrix, the more eigenfrequencies can be calculated.

The generalized eigenvalue problem (7.44) for the Stokes flow model has to be solved iteratively, due to the complexity of the integral. To this end, we split the integral into three parts, so that the equation to be solved becomes

$$
\begin{aligned}
& 2 P \sum_{k=1}^{\infty} \frac{c_{k}}{j_{k} j_{n}} J_{1}\left(j_{k}\right) J_{1}\left(j_{n}\right)+\frac{1}{2} c_{n} j_{n}^{2} J_{1}^{2}\left(j_{n}\right)= \\
& \sum_{k=1}^{\infty} c_{k} j_{k} j_{n} J_{1}\left(j_{k}\right) J_{1}\left(j_{n}\right)\left[\zeta^{2} f\left(j_{k}, j_{n}\right)+\zeta \mathrm{Oh}\left\{g\left(j_{k}, j_{n}\right)+h\left(j_{k}, j_{n}, \zeta, \mathrm{Oh}\right)\right\}\right],
\end{aligned}
$$


with $f\left(j_{k}, j_{n}\right)$ given by (7.56), $g\left(j_{k}, j_{n}\right)$ by (7.71), and $h\left(j_{k}, j_{n}\right)$ by (7.72). To reduce (7.73) to a generalized eigenvalue problem that can be solved with Mathematica, we write $\mathbf{d}=\zeta \mathbf{c}$, so that the resulting system becomes

$\left(\begin{array}{cc}\emptyset & \mathrm{I} \\ 2 P \mathrm{~B}+\frac{1}{2} \mathrm{C} & \mathrm{Oh}\left[\mathrm{G}+\mathrm{H}\left(\zeta^{(\mathrm{i})}, \mathrm{Oh}\right)\right]\end{array}\right)\left(\begin{array}{l}\mathbf{c}^{(i+1)} \\ \mathbf{d}^{(i+1)}\end{array}\right)=\zeta^{(i+1)}\left(\begin{array}{cc}\mathrm{I} & \emptyset \\ \emptyset & -\mathrm{A}\end{array}\right)\left(\begin{array}{c}\mathbf{c}^{(i+1)} \\ \mathbf{d}^{(i+1)}\end{array}\right)$,

with $i$ the iteration number, $\mathbf{c}$ a vector with elements $c_{k}, \emptyset$ the zero matrix , I the unit matrix, $A$ given by (7.58), $B$ by (7.59), $C$ by (7.60), $G$ by (7.74), and $H$ by (7.75). The matrices $A, G$, and $\mathrm{H}$ correspond to the three terms in which the integral in (7.44) is decomposed. The generalized eigenvalue problem (7.46) is solved iteratively with the modified potential-flow solution used as initial guess $\zeta^{(1)}$ in matrix $\mathrm{H}$. With this initial guess, we evaluate the integral (7.72) numerically, which we then use to solve (7.46). This step permits an improved estimate $\zeta^{(2)}$ of the eigenvalue, which is substituted again into the matrix $\mathrm{H}$. This procedure is repeated until convergence is reached, i.e. until the difference in both frequency and damping between the current and the previous iteration is less than $0.01 \%$ of the current result. Again, we investigated the influence of the matrix size on the calculation of the eigenfrequency and interface shape for the lowest three modes by taking a system size up to $K=16$. For the lowest two modes, $K=3$ was sufficiently accurate to calculate the eigenfrequency, whereas for mode $3, K=6$ was used. Convergence of (7.46) was achieved after 4 iterations for the three lowest modes; see Appendix A.2 for details. To further check the convergence of the solutions obtained, we used the potential flow solution as initial guess, and slowly increased the Ohnesorge number from 0 to $\mathrm{Oh}_{s}=0.0303$. Using this method, we obtained the same results for the resonance frequency and the damping as by starting directly at $\mathrm{Oh}_{s}$ with the modified potential flow solution as initial guess.

\subsection{Results}

In the generalized eigenvalue problem for $\zeta, \eta$, only two dimensionless parameters appear: the ratio of the gas stiffness to the surface tension stiffness, $P(7.21)$, and the Ohnesorge number Oh (7.28). For a gas pocket with $\lambda=1$ in a $15-\mu \mathrm{m}$ cylindrical micropit submerged in water under standard conditions, the corresponding values of the dimensionless groups are $P=P_{s}=20.5$ and $\mathrm{Oh}=\mathrm{Oh}_{s}=0.0303$. In this example, we find the dimensional resonance frequency for the first three modes in potential flow to be 121,274 , and $556 \mathrm{kHz}$, respectively. The first result is not very different from the frequency estimate (7.1) by Miller \& Nyborg [14] which is $151 \mathrm{kHz}$ for the lowest mode of a $15-\mu \mathrm{m}$ pit. The interface shape for the first three modes is depicted 
(a)

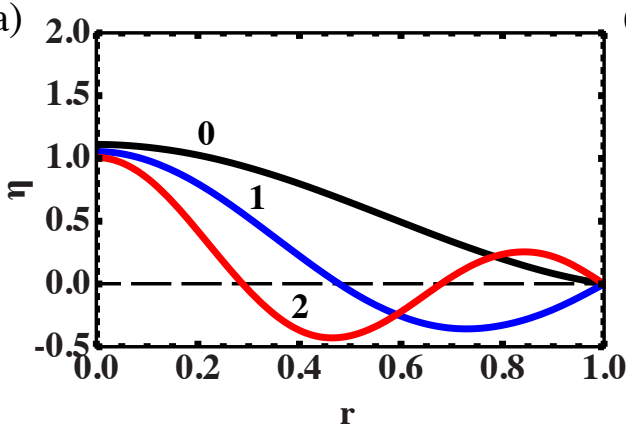

(b)

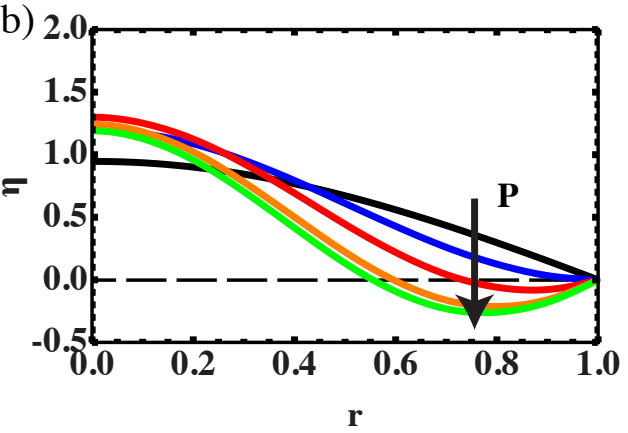

Figure 7.2: The interface shape as calculated from the potential flow model for (a) $P=20.5$, mode 0 (black), mode 1 (blue), and mode 2 (red). (b) The interface shape for mode 0 with $P=0$ (black), 40 (blue), 80 (red), 160 (orange), 320 (green). Note that for $P \geq 80$, mode 0 has an extra node in addition to the one at the rim of the pit.

in Fig. 7.2(a). One can see that with these parameter values the largest contribution to the interface shape of mode 0 comes from the first term in the Fourier-Bessel series (7.25), whereas for mode 1 the largest contribution comes from the second term, etc.

Figures 7.3, 7.4, and 7.5 show how the resonance frequency and damping coefficient depend on the two dimensionless parameters $P$ and $\mathrm{Oh}$. As expected, both the damping and frequency increase with the mode number with the result that, after a generic initial perturbation, the bubble will oscillate the longest at its fundamental resonance frequency whereas higher frequencies dampen out earlier. The difference in resonance frequency between the potential flow (PF), modified potential flow $(\mathrm{mPF})$, and Stokes flow (SF) models is very small which means that, in the parameter range of interest, the resonance frequency is mainly determined by inertia and can be obtained from the potential flow model with sufficient accuracy.

In Fig. 7.3(a), the graph of the frequency $f=\omega / 2 \pi$ versus $P$ for mode 0 shows that the resonance frequency first increases with $P$, until it levels off to a dimensionless value $f=1.72$ for $P>200$, approximately. In the approximate solution (7.1) by Miller [14] such a plateau is not observed. Figure 7.4(a) shows graphs of $f$ versus $P$ for modes 1 and 2. Here, a similar increase in $f$ with $P$ is observed, but the plateau is reached at larger values of $P$. Initially, the resonance frequency increases with $P$ because at larger $P$ it becomes more difficult to change the volume of the gas, and hence the system becomes stiffer, which leads to a higher resonance frequency. The reason for the occurrence of a plateau in the frequency lies in the increasing stiffness 
(a)

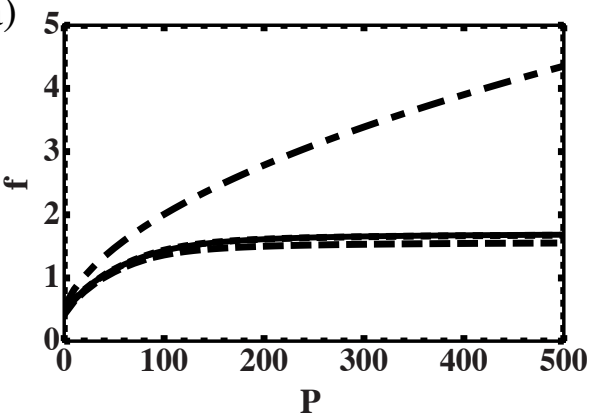

(b)

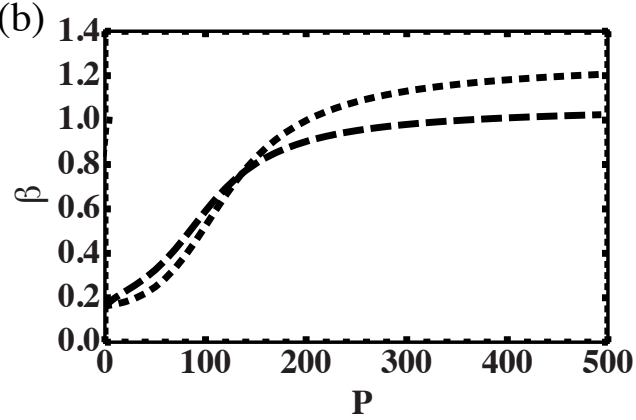

Figure 7.3: (a) The dimensionless frequency $f=\omega / 2 \pi$ of mode 0 versus the dimensionless parameter $P$, defined in (7.21), for potential flow (black, solid), modified potential flow (black, dotted) and Stokes flow (black, dashed) with $\mathrm{Oh}=0.0303$. The results for potential flow and modified potential flow nearly overlap. For comparison, the result of Miller \& Nyborg [14] (7.1) is also shown (dash-dotted). (b) Dimensionless damping $\beta$ versus $P$ for the modified potential flow and Stokes flow models.

(a)

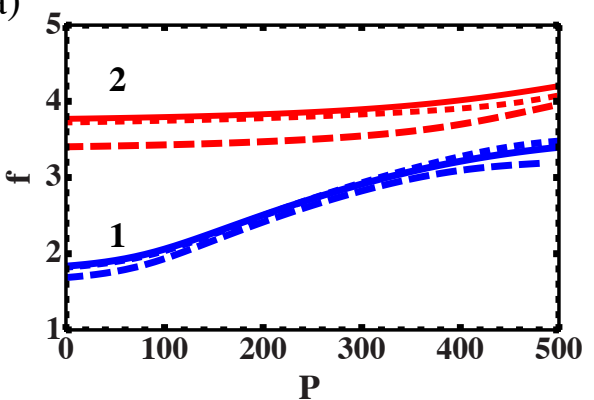

(b)

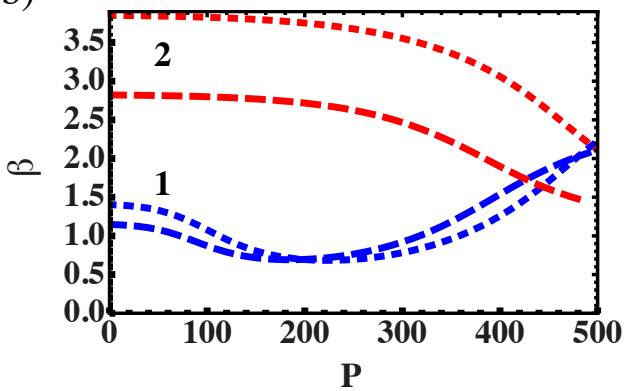

Figure 7.4: (a) The dimensionless frequency $f$ of mode 1 (blue), and mode 2 (red), versus the dimensionless parameter $P$, defined in (7.21), for potential flow (solid), modified potential flow (dotted) and Stokes flow (dashed) with $\mathrm{Oh}=0.0303$. A plateau in the frequencies similar to that for mode 0 is observed, but it occurs at larger $P$ (not shown in the figure for mode 2). (b) Dimensionless damping $\beta$ versus $P$ for the different cases. 
(a)

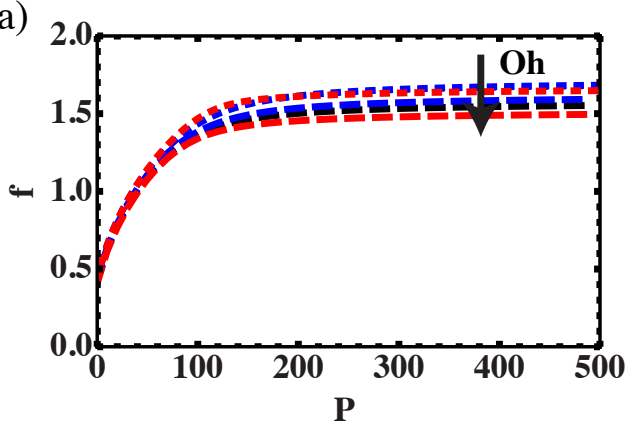

(b)

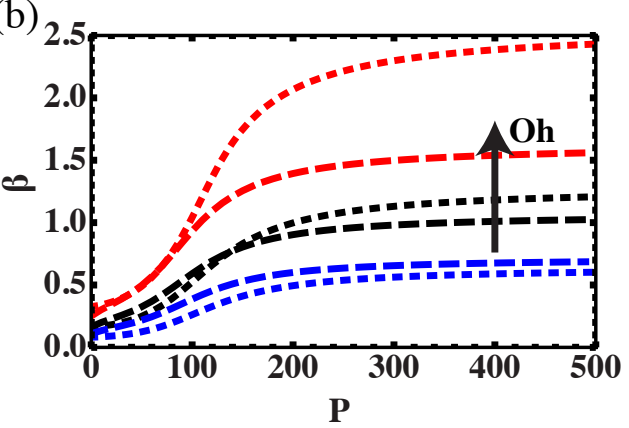

Figure 7.5: (a) The dimensionless frequency $f$ of mode 0 versus the dimensionless parameter $P$, defined in (7.21), for modified potential flow (dotted), and Stokes flow (dashed) with $\mathrm{Oh}=0.01515$ (blue), $\mathrm{Oh}=0.0303$ (black), and $\mathrm{Oh}=0.0606$ (red). (b) Dimensionless damping $\beta$ versus $P$ for the different cases.

of the gas. As $P$ increases and the system becomes stiffer, it becomes more difficult to decrease the gas volume change $H$, as defined in (7.13), and the system responds by increasing the area of the interface instead (see Fig. 7.2(a)). To further decrease the volume change, at some point an extra node has to appear in the interface shape, as can be seen in Fig. 7.2(a) for $P \geq 80$. This node is pushed towards the axis of the pit as $P$ is increased further. In this way, the interface area increases more and more, and the net volume change due to the surface elevation eventually tends to zero, which means that $V \rightarrow V_{0}$. Figure 7.6 shows that this decrease in the amplitude of the volume oscillations occurs in such a way that the product $P H$ tends to a constant value. Hence, the resonance frequency levels off, and the system eventually oscillates with a fixed interface shape. The interface shapes corresponding to modes 1 and 2 are depicted in Fig. 7.7. For mode 1, the extra node appears around $P \simeq 500$, whereas for mode 2, it will occur at a larger $P$.

The parameter $P$ also has an effect on the damping coefficient as shown in Figs. 7.3(b) and 7.4(b). For mode 0 the damping coefficient increases with $P$, until a final plateau is reached. For mode 1, however, a minimum is observed and the plateau is reached for larger $P$. For mode 2, the damping decreases and reaches a minimum beyond the maximum value of $P$ shown in the graph. The presence of a minimum could be explained as follows: as the stiffness of the gas increases with $P$, the relative volume change decreases, which leads to a smaller liquid displacement and viscous energy dissipation. However, as the frequency increases, the damping increases as well. These two effects compete, and give rise to a minimum in the damping coefficient. The plateau is reached at larger $P$, when the product $P H$ tends to a finite value 


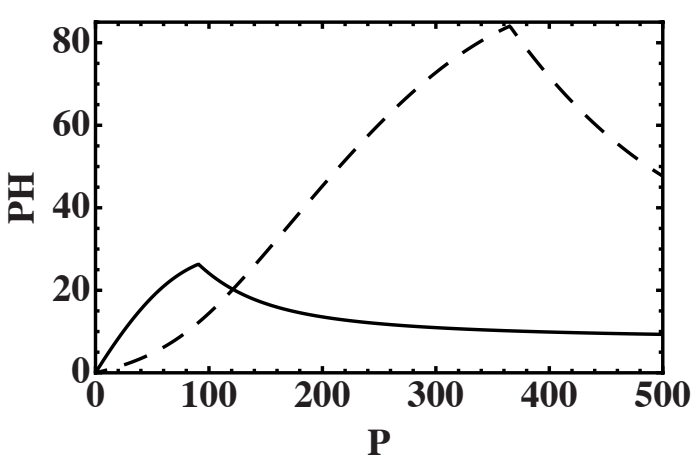

Figure 7.6: The relative stiffness of the gas pocket times its volume change, $P H$, versus $P$, for mode 0 (solid) and mode 1 (dashed). Initially $P H$ increases, until a maximum is reached, then it tends to a finite value.

(see also Fig. 7.6). The difference in damping coefficients between the mPF and SF models is larger for the higher modes. One also observes that, for some values of $P$, the mPF damping is even larger than the SF damping. This behaviour is due to the difference in velocity profiles between the $\mathrm{mPF}$ and SF, and is also known to occur for shape oscillations of drops and bubbles [19].

Whereas $P$ has a large influence on the resonance frequency of the pit, the influence of $\mathrm{Oh}$ is only very small as shown in Fig. 7.5. The influence of the Ohnesorge number on the damping coefficient is of course large.

\subsection{Conclusion}

The resonance frequency, damping and interface shape of a gas pocket entrapped on the surface of an submerged solid have been calculated. To describe the hydrodynamic problem in the liquid domain, both a potential and an unsteady Stokes flow model have been used. The potential flow model gives a reliable prediction of the resonance frequency of the gas pocket, which is mainly determined by inertia in the liquid. To derive an estimate for the damping of the oscillations, the bulk dissipation was calculated from the potential flow model. A more accurate prediction of the damping was derived based on the unsteady Stokes flow model, which is valid throughout entire domain and therefore includes the contributions of both the boundary layer and the bulk. However, the Stokes flow results will overestimate the real damping somewhat in the case that the liquid-gas interface is clean: in the method 

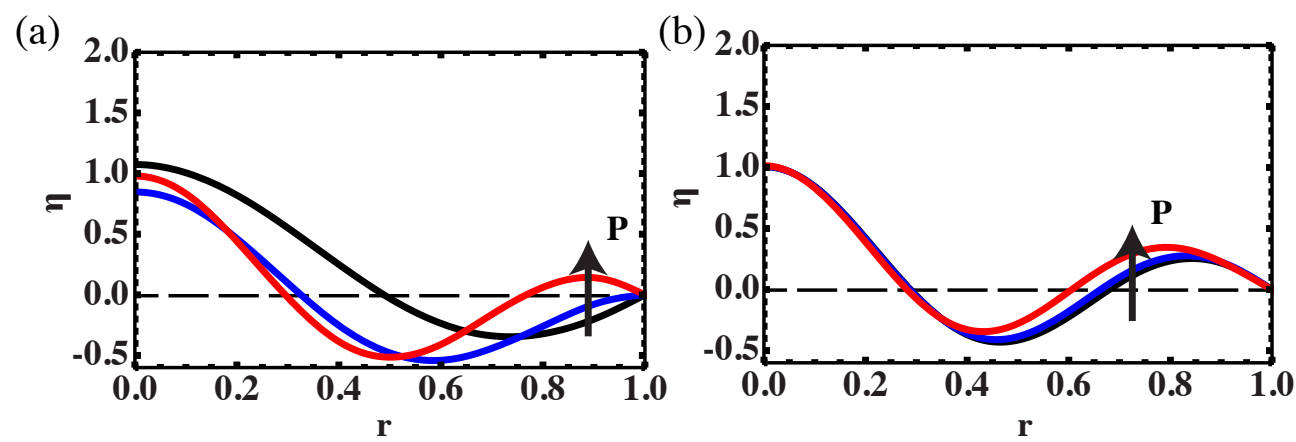

Figure 7.7: The interface shape as calculated from the potential flow model with $\mathrm{Oh}=0.0303$ for $P=0$ (black), $P=250$ (blue), and $P=500$ (red) for (a) mode 1 and (b) mode 2. Note that, for $P=500$, mode 1 has developed an extra node.

described here, a no-slip condition on the free surface was used, which leads to some additional dissipation.

The resonance frequency, damping and interface shape of an entrapped gas pocket depend on two dimensionless numbers: the ratio $P$ of the gas stiffness to the surface tension stiffness, defined in (7.21), and the Ohnesorge number (7.28), which represents the relative importance of viscous forces. In general, the resonance frequency increases with increasing gas stiffness. However, an unexpected feature of our results is that when the volume stiffness of the gas pocket greatly exceeds the surface stiffness, the normal modes develop an extra node and the resonance frequency tends to an asymptotic value.

\section{Appendix}

\section{A.1 Potential flow solution}

To obtain a solution for the velocity potential $\phi$ we set

$$
\partial_{z} \phi=\int_{0}^{\infty} k \Phi(k) J_{0}(k r) e^{-k z} \mathrm{~d} k
$$

and, upon integration, find that

$$
\phi=-\int_{0}^{\infty} \Phi(k) J_{0}(k r) e^{-k z} \mathrm{~d} k .
$$


Using the boundary conditions (7.17) and (7.18) and the orthogonality relation for Bessel functions we obtain

$$
\int_{0}^{\infty} \int_{0}^{\infty} k r \Phi(k) J_{0}(k r) J_{0}(h r) \mathrm{d} r \mathrm{~d} k=\int_{0}^{\infty} \Phi(k) \delta(h-k) \mathrm{d} k=\zeta \int_{0}^{1} \eta(r) r J_{0}(h r) \mathrm{d} r,
$$

and, therefore,

$$
\Phi(h)=\zeta \int_{0}^{1} \eta(r) r J_{0}(h r) \mathrm{d} r=\zeta v(h)
$$

Thus

$$
\phi(r, 0)=-\zeta \int_{0}^{\infty} v(k) J_{0}(k r) \mathrm{d} k .
$$

Substituting this result into (7.20) we find (7.22).

The next step is to express the interface deformation $\eta$ in terms of the FourierBessel series (7.25), to obtain

$$
\begin{gathered}
v(s)=\sum_{k=1}^{\infty} c_{k} \int_{0}^{1} r J_{0}(s r) J_{0}\left(j_{k} r\right) \mathrm{d} r=\sum_{k=1}^{\infty} c_{k} \frac{j_{k} J_{0}(s) J_{1}\left(j_{k}\right)}{j_{k}^{2}-s^{2}}, \\
H=2 \sum_{k=1}^{\infty} c_{k} \int_{0}^{1} r J_{0}\left(j_{k} r\right) \mathrm{d} r=2 \sum_{k=1}^{\infty} \frac{c_{k}}{j_{k}} J_{1}\left(j_{k}\right) .
\end{gathered}
$$

Substitution into (7.24) leads to

$$
\sum_{k=1}^{\infty} c_{k} J_{0}\left(j_{k} r\right)=\frac{1}{2} \mathrm{P} \sum_{k=1}^{\infty} \frac{c_{k}}{j_{k}} J_{1}\left(j_{k}\right)\left(r^{2}-1\right)+\zeta^{2} \sum_{k=1}^{\infty} c_{k} j_{k} J_{1}\left(j_{k}\right) \int_{0}^{\infty} \frac{\left[J_{0}(s)-J_{0}(s r)\right] J_{0}(s)}{\left(j_{k}^{2}-s^{2}\right) s^{2}} \mathrm{~d} s .
$$

To obtain an equation for each of the unknowns, we multiply (7.54) by $r J_{0}\left(j_{n} r\right)$ and integrate between 0 and 1 , to find

$$
\begin{aligned}
& 2 P \sum_{k=1}^{\infty} \frac{c_{k}}{j_{k} j_{n}} J_{1}\left(j_{k}\right) J_{1}\left(j_{n}\right)+\frac{1}{2} c_{n} j_{n}^{2} J_{1}^{2}\left(j_{n}\right) \\
& =-\zeta^{2} \sum_{k=1}^{\infty} c_{k} j_{k} J_{1}\left(j_{k}\right) \int_{0}^{\infty} \frac{J_{0}(s)}{j_{k}^{2}-s^{2}} \mathrm{~d} s \int_{0}^{1} r J_{0}(s r) J_{0}\left(j_{n} r\right) \mathrm{d} r \\
& =-\zeta^{2} \sum_{k=1}^{\infty} c_{k} J_{1}\left(j_{k}\right) j_{k} j_{n} J_{1}\left(j_{n}\right) f\left(j_{k}, j_{n}\right),
\end{aligned}
$$

with

$$
\begin{aligned}
f\left(j_{k}, j_{n}\right) & =\int_{0}^{\infty} \frac{J_{0}^{2}(s)}{\left(s^{2}-j_{k}^{2}\right)\left(s^{2}-j_{n}^{2}\right)} d s \\
& =\left\{\begin{array}{cc}
\frac{4}{\pi} \frac{1}{j_{k}^{2}-j_{n}^{2}}\left[{ }_{2} F_{3}\left(1,1 ; \frac{3}{2}, \frac{3}{2}, \frac{3}{2} ;-j_{n}^{2}\right)-{ }_{2} F_{3}\left(1,1 ; \frac{3}{2}, \frac{3}{2}, \frac{3}{2} ;-j_{k}^{2}\right)\right], & \text { if } k \neq n, \\
\frac{32}{27 \pi} 2 F_{3}\left(2,2 ; \frac{5}{2}, \frac{5}{2}, \frac{5}{2} ;-j_{k}^{2}\right), & \text { if } k=n,
\end{array}\right.
\end{aligned}
$$


where ${ }_{2} F_{3}$ is the hypergeometric function [20]. After truncation of the Fourier-Bessel series (7.25) to $K$ terms, we can express the eigenvalue problem in matrix form as

$$
\left(\zeta^{2} \mathrm{~A}+2 P \mathrm{~B}+\frac{1}{2} \mathrm{C}\right) \mathbf{c}=\mathbf{0},
$$

with A a $K \times K$-matrix with coefficients

$$
A_{k n}=J_{1}\left(j_{k}\right) J_{1}\left(j_{n}\right) j_{k} j_{n} f\left(j_{k}, j_{n}\right),
$$

B a $K \times K$-matrix with coefficients

$$
B_{k n}=\frac{J_{1}\left(j_{k}\right) J_{1}\left(j_{n}\right)}{j_{k} j_{n}},
$$

and $\mathrm{C}$ a $K \times K$ diagonal matrix with coefficients

$$
C_{k n}=j_{k}^{2} J_{1}^{2}\left(j_{k}\right) \delta_{k n},
$$

and c a $K$-array with coefficients $c_{k}$. The generalized eigenvalue problem (7.57) is the solved with Mathematica 8 (Wolfram Research).

\section{A.2 Stokes flow solution}

The general solution to (7.41) in terms of the Hankel transform reads

$$
\Omega(r, z)=\int_{0}^{\infty} G(k) J_{1}(k r) e^{-z \sqrt{k^{2}+\zeta / \mathrm{Oh}}} \mathrm{d} k,
$$

with $G$ to be determined from the boundary conditions (7.4), (7.5), (7.6), and (7.8). From (7.61) we can determine an expression for stream function $\Psi$ using

$$
\boldsymbol{\Omega}=-\nabla^{2}\left(\frac{\Psi}{r} \boldsymbol{e}_{\theta}\right) .
$$

The homogeneous solution of (7.62) reads

$$
\Psi_{h}(r, z)=r \int_{0}^{\infty} F(k) J_{1}(k r) e^{-k z} \mathrm{~d} k,
$$

with $F$ to be determined from the boundary conditions. The particular solution can be found from (7.41):

$$
\boldsymbol{\Omega}=-\nabla^{2}\left(\frac{\Psi_{p}}{r} \boldsymbol{e}_{\theta}\right)=\frac{\mathrm{Oh}}{\zeta} \nabla^{2} \boldsymbol{\Omega},
$$


and hence

$$
\Psi_{p}(r, z)=-r \frac{\mathrm{Oh}}{\zeta} \int_{0}^{\infty} G(k) J_{1}(k r) e^{-z \sqrt{k^{2}+\zeta / \mathrm{Oh}}} \mathrm{d} k .
$$

Once we know $\Psi=\Psi_{h}+\Psi_{p}$, we can find expressions for the velocity field

$$
\begin{aligned}
& u_{r}(r, z)=\int_{0}^{\infty} k F(k) J_{1}(k r) e^{-k z} \mathrm{~d} k-\frac{\mathrm{Oh}}{\zeta} \int_{0}^{\infty} \sqrt{k^{2}+\zeta / \mathrm{Oh}} G(k) J_{1}(k r) e^{-z \sqrt{k^{2}+\zeta / \mathrm{Oh}}} \mathrm{d} k, \\
& u_{z}(r, z)=\int_{0}^{\infty} k F(k) J_{0}(k r) e^{-k z} \mathrm{~d} k-\frac{\mathrm{Oh}}{\zeta} \int_{0}^{\infty} k G(k) J_{0}(k r) e^{-z \sqrt{k^{2}+\zeta / \mathrm{Oh}}} \mathrm{d} k
\end{aligned}
$$

Expressions for $F$ and $G$ can now be obtained from (7.4), (7.5), (7.6), and (7.8)

$$
\begin{aligned}
& F(k)-\frac{\mathrm{Oh}}{\zeta} G(k)=\zeta v(k), \\
& k F(k)-\frac{\mathrm{Oh}}{\zeta} G(k) \sqrt{k^{2}+\zeta / \mathrm{Oh}}=0,
\end{aligned}
$$

with $v(k)$ given by (7.23), which give

$$
\begin{aligned}
G(k) & =v(k)\left(k^{2}+k \sqrt{k^{2}+\zeta / \mathrm{Oh}}\right) \\
F(k) & =v(k)\left[\zeta+\frac{\mathrm{Oh}}{\zeta}\left(k^{2}+k \sqrt{k^{2}+\zeta / \mathrm{Oh}}\right)\right] .
\end{aligned}
$$

The pressure in the liquid can now be obtained from (7.39). Using (7.40) and (7.41) one finds that

$$
\nabla p=-\zeta\left(\nabla \times \frac{\Psi_{h}}{r} \boldsymbol{e}_{\theta}\right)
$$

and hence the liquid pressure reads

$$
p(r, z)=\zeta \int_{0}^{\infty} F(k) J_{0}(k r) e^{-k z} \mathrm{~d} k .
$$

This results evaluated at $z=0$ is then substituted into (7.42) to find (7.43) by integration. Substitution of the Fourier-Bessel series (7.25) results in (7.44).

Due to the complexity of the integral in (7.44), the system has to be solved iteratively. To this end, we split the integral into parts. The resulting equation is given by (7.46) with $f$ given by (7.56), $g$ by

$$
\begin{aligned}
g\left(j_{k}, j_{n}\right) & =\int_{0}^{\infty} \frac{s^{2} J_{0}^{2}(s)}{\left(s^{2}-j_{k}^{2}\right)\left(s^{2}-j_{n}^{2}\right)} \mathrm{d} s \\
& =\left\{\begin{array}{cc}
\frac{4}{\pi} \frac{1}{j_{k}^{2}-j_{n}^{2}}\left[j_{n 2}^{2} F_{3}\left(1,1 ; \frac{3}{2}, \frac{3}{2}, \frac{3}{2} ;-j_{n}^{2}\right)-j_{k}^{2} F_{3}\left(1,1 ; \frac{3}{2}, \frac{3}{2}, \frac{3}{2} ;-j_{k}^{2}\right)\right], & \text { if } k \neq n, \\
-\frac{4}{\pi} 2 F_{3}\left(1,2 ; \frac{3}{2}, \frac{3}{2}, \frac{3}{2} ;-j_{k}^{2}\right), & \text { if } k=n,
\end{array}\right.
\end{aligned}
$$



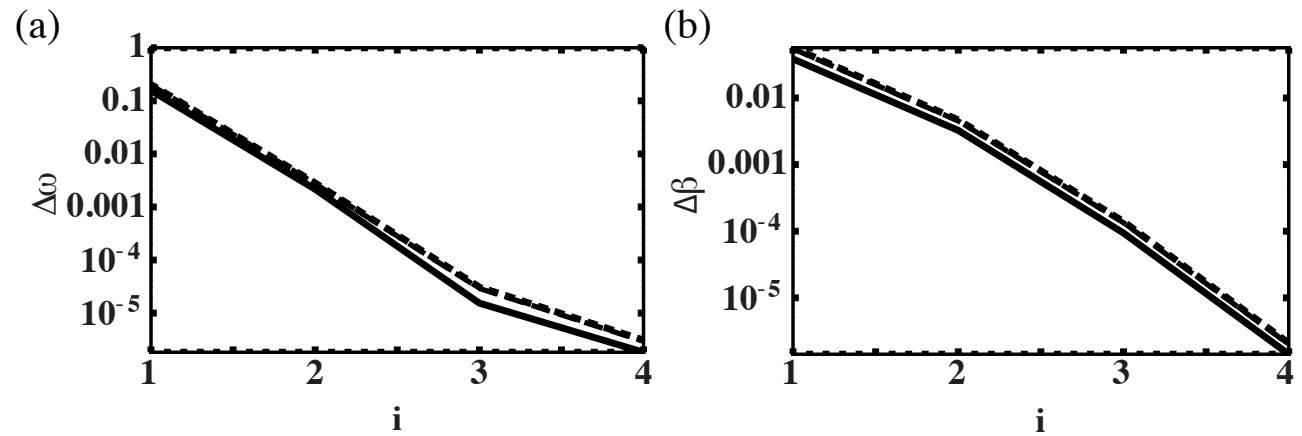

Figure 7.8: (a) The increment in angular frequency $\omega$ of mode 0 versus iteration number $i$ illustrating convergence of the solution process. (b) Increment of damping $\beta$ versus iteration number $i$. The solution is shown for different matrix sizes: $1 \times 1$ (solid) $2 \times 2$ (dashed) $3 \times 3$ (dotted).

and $h$ by

$$
h\left(j_{k}, j_{n}, \zeta, \mathrm{Oh}\right)=\int_{0}^{\infty} \frac{s J_{0}^{2}(s)}{\left(j_{k}^{2}-s^{2}\right)\left(j_{n}^{2}-s^{2}\right)} \sqrt{s^{2}+\zeta / \mathrm{Oh}} \mathrm{d} s .
$$

After truncation of the Fourier-Bessel series to $K$ terms, the resulting equation (7.46) in matrix form becomes

$$
\left\{\left(\zeta^{(i+1)}\right)^{2} \mathrm{~A}+2 \mathrm{~PB}+\frac{1}{2} \mathrm{C}+\zeta^{(i+1)} \mathrm{Oh}\left[\mathrm{G}+\mathrm{H}\left(\zeta^{(i)}, \mathrm{Oh}\right)\right]\right\} \mathbf{c}^{(i+1)}=\mathbf{0},
$$

with $i$ the iteration number, A given by (7.58), B given by (7.59), and $\mathrm{C}$ given by (7.60); $\mathrm{G}$ is a $K \times K$-matrix with coefficients

$$
G_{k n}=J_{1}\left(j_{k}\right) J_{1}\left(j_{n}\right) j_{k} j_{n} g\left(j_{k}, j_{n}\right),
$$

and $\mathrm{H}$ is a $K \times K$-matrix with coefficients

$$
H_{k n}=J_{1}\left(j_{k}\right) J_{1}\left(j_{n}\right) j_{k} j_{n} h\left(j_{k}, j_{n}, \zeta^{(i)}, \mathrm{Oh}\right) .
$$

Figure 7.8 shows that for mode 0 , convergence is reached within 4 iterations, irrespective of the matrix size.

\section{References}

[1] D. L. Miller, "Frequency relationships for ultrasonic activation of free microbubbles, encapsulated microbubbles, and gas-filled micropores", J. Acoust. Soc. Am. 104, 2498-2505 (1998). 
[2] L. A. Kuznetsova, S. Khanna, N. N. Amso, W. T. Coakley, and A. A. Doinikov, "Cavitation bubble-driven cell and particle behavior in an ultrasound standing wave", J. Acoust. Soc. Am. 117, 104-112 (2005).

[3] P. V. Zinin and J. S. Allen, "Deformation of biological cells in the acoustic field of an oscillating bubble", Phys. Rev. E 79, 021910 (2009).

[4] M. Postema and O. H. Gilja, "Ultrasound-directed drug delivery", Curr. Pharm. Biotechnol. 8, 335-361 (2007).

[5] R. J. Browning, H. Mulvana, M.-X. Tang, J. V. Hajnal, D. J. Wells, and R. J. Eckersley, "Effect of albumin and dextrose concentration on ultrasound and microbubble mediated gene transfection in vivo", Ultrasound Med. Biol. 38, 1067-1077 (2012).

[6] R. H. Liu, J. N. Yang, M. Z. Pindera, M. Athavale, and P. Grodzinski, "Bubbleinduced acoustic micromixing", Lab on a Chip 2, 151-157 (2002).

[7] D. F. Rivas, A. Prosperetti, A. G. Zijlstra, D. Lohse, and H. J. G. E. Gardeniers, "Efficient sonochemistry through microbubbles generated with micromachined surfaces", Angew. Chem. Int. Edit. 49, 9699-9701 (2010).

[8] N. Bremond, M. Arora, C.-D. Ohl, and D. Lohse, "Controlled multibubble surface cavitation”, Phys. Rev. Lett. 96, 224501 (2006).

[9] B. M. Borkent, S. Gekle, A. Prosperetti, and D. Lohse, "Nucleation threshold and deactivation mechanisms of nanoscopic cavitation nuclei", Phys. Fluids 21, 102003 (2009).

[10] D. L. Miller, "Experimental investigation of the response of gas-filled micropores to ultrasound", J. Acoust. Soc. Am. 71, 471-476 (1982).

[11] E. A. Neppiras, W. L. Nyborg, and D. L. Miller, "Non-linear behavior and stability of trapped micron-sized cylindrical gas bubbles in an ultrasonic field", Ultrasonics 21, 109-115 (1983).

[12] H. Rathgen, K. Sugiyama, C.-D. Ohl, D. Lohse, and F. Mugele, "Nanometerresolved collective micromeniscus oscillations through optical diffraction", Phys. Rev. Lett. 99, 214501 (2007).

[13] M. S. Plesset and A. Prosperetti, "Bubble dynamics and cavitation", Ann. Rev. Fluid Mech. 9, 145-185 (1977). 
[14] D. L. Miller and W. L. Nyborg, "Theoretical investigation of the response of gas-filled micropores and cavitation nuclei to ultrasound", J. Acoust. Soc. Am. 73, 1537-1544 (1983).

[15] B. Cuenot, J. Magnaudet, and B. Spennato, "The effects of slightly soluble surfactants on the flow around a spherical bubble", J. Fluid Mech. 339, 25-53 (1997).

[16] G. K. Batchelor, An introduction to fluid dynamics (Cambridge university press) (2000).

[17] L. D. Landau and E. M. Lifshitz, Fluid mechanics, 2nd edition (Elsevier) (2004).

[18] H. Lamb, Hydrodynamics, 6 edition (Dover publications) (1945).

[19] A. Prosperetti, "Normal-mode analysis for the oscillations of a viscous liquid drop in an immiscible liquid", J. Mec. 19, 149-181 (1980).

[20] M. Abramowitz and I. A. Stegun, Handbook of mathematical functions (Dover, New York) (1968). 


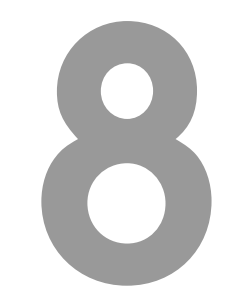

\section{Summary and Outlook}

When a suspension drop evaporates, it leaves behind a drying stain. In order to either prevent ring-stain formation or to be able to control and use the patterns formed by drying drops, one needs to understand the basic physics of evaporating drops and their internal fluid flow. In this thesis we focused on the fundamentals of drop evaporation, evaporation-driven flow in drying drops, and the subsequent particle transport and deposition. We used a simple model system: a macroscopic sessile water drop that evaporates under atmospheric conditions and contains spherical polystyrene particles.

In Chapter 2 we studied the evaporation of water drops on superhydrophobic carbon nanofibre substrates. On these substrates, the contact line of a drop remains pinned, and hence the evaporation occurs in the constant contact-area mode. We found that the evolution of the rate of mass loss and contact angle of the drops is accurately described by a diffusion-based model for the entire range of contact angles $\left(0-150^{\circ}\right)$. Hence, the evaporation of macroscopic drops from these substrates under atmospheric conditions is quasi-static, diffusion-driven and thermal effects play no role. Furthermore, we have shown that evaporation occurs in a universal way for all drop sizes and initial contact angles.

The evaporative flux from the drop surface drives a flow inside the drying drop. The nature of this flow close to the contact line of the drop has been addressed in Chapter 3. We derived self-similar solutions to the Stokes flow near the contact line, by approximating the local drop shape by a wedge geometry. We found that there are three mechanisms that can drive the flow in the wedge: the evaporative flux from the 
drop surface, the downward motion of the liquid-air interface when the contact line is pinned, and an eigenmode contribution that satisfies the homogeneous boundary conditions and can give rise to viscous corner eddies. Which of the three mechanisms is asymptotically dominant depends on the contact angle: below a critical angle of $133.4^{\circ}$ the evaporative-flux driven solution dominates, for larger angles the eigenmode flow is dominant. We found that for small contact angles $\left(\theta \ll 90^{\circ}\right)$ the velocity field in the entire drop is accurately described in the lubrication approximation, all the way down to the contact line where the evaporative flux diverges. For larger contact angles, the flow structure becomes more complicated and the lubrication model can no longer be applied. For contact angles larger than $126^{\circ}$ we even observed sequences of wedge-like flow structures in the drop. In order to know the size of these convection rolls and to determine the strength of the eigenmode flow, a numerical simulation of the flow in the entire drop would have to be performed.

In Chapter 4 we consider an evaporating drop on a liquid subphase, on which the contact line is moving. For pinned contact lines, the downward motion of the liquidair interface gives rise to a flow. On a liquid subphase however, there is a contribution from the receding motion of the contact line. We found that this moving contactline contribution dominates both the evaporative-flux driven flow and the eigenmode flow when the contact angle between the drop and the horizontal surface of the liquid subphase is smaller than $90^{\circ}$. In contrast to a drop on a solid substrate, the eigenmode contribution to the flow on a liquid subphase is found to be always subdominant. The flow in the evaporating drop also generates a flow in the liquid subphase. The ratio of viscosities of the two liquids determines the coupling between the two flows. We observed sequences of wedge-like flow structures similar to those found in a drying drop on a solid. However, these flow structures are not only present in the drop itself but also in the liquid subphase.

A fourth contribution to the flow in a drying drop can be generated by Marangoni stresses at the liquid-air interface. These stresses arise from temperature -and hence surface tension-gradients on the liquid-air interface, due to the non-uniform evaporative flux from the drop surface. The resulting Marangoni flow adds to the evaporationdriven flow. We analysed the importance of the Marangoni flow in the asymptotic region close to the contact line, and found that sufficiently close to the contact line the Marangoni effect is subdominant and the evaporation-driven flow always wins. However, the cross-over length scale is of the order of $10 \mathrm{~nm}$ for a millimetre-sized drop with a contact angle of order unity. This means that the Marangoni-effect should significantly alter the velocity field inside the drying drop. One can add the Marangoni flow to the other flow solutions in the wedge following the method of Ristenpart et al. [1]. For water drops, however, the Marangoni effect has not been observed ex- 
perimentally $[2,3]$. This absence of Marangoni flow in drying water drops is still not entirely understood, but could be due to the presence of surfactants [2].

The nature of the flow inside a drying colloidal suspension drop determines the structure of the drying stains. When the contact line of the drying drop is pinned, ring-shaped stains form during drop evaporation. In Chapter 5 we studied the dynamics of this ring-stain formation, and showed that the structure of the drying stain originates from a competition between particle diffusion and convection. We used the lubrication approximation to model the flow inside the drying drop, and found that the enormous increase in particle speed during the last moments of the drop life is caused by the decreasing drop height. Particles that arrive at the contact line during this rush-hour phase form a disordered pattern, whereas particles that arrive earlier, at low speed, form highly ordered crystalline patterns. Within this crystalline phase, we observed transitions from hexagonal to square particle packings, which we attributed to the confinement of the particles by the glass slide and the liquid-air interface. By controlling not only the particle size but also the evaporation rate and contact angle of the drop, and hence the internal flow, one can use evaporation to generate large-scale colloidal crystals.

Three-dimensional ordered structures of colloidal particles were obtained in Chapter 6 , where we studied drying drops with moving contact lines. We used a special type of superhydrophobic substrate that consists of pillars with some overhang, so that the drops remain in the Cassie-Baxter state during the entire evaporation process. The evaporation of drops from these substrates resulted in soccer-ball like spherical structures that consist of highly-ordered colloidal particles. Similar to the coffee stains studied in Chapter 5, we found that the particle ordering in these structures is determined by the competition between particle diffusion and convection. The convection in this case is caused by the inward motion of the liquid-air interface. We have shown that the ordering of the particles in the soccer balls depends on the number of particles in the system, and the diffusion constants for the particles in the liquid and for the vapour in the surrounding air. The final structures that form can hence be controlled by tuning the evaporation rate of the drops.

The three-dimensional arrangement of the particles inside the microscopic soccer balls is not entirely understood. In this system, the liquid-air interface "collects" the colloidal particles while moving inwards. Hence, one expects a shell of particles to form on the surface of the drop. When the drop looses volume due to the evaporation, this shell could buckle, which would lead to hollow structures [4]. The soccer balls we find are entirely solid and show a very high packing fraction, which indicates there are no cavities inside. At present, it is not understood what happens to the particles once they collect at the liquid-air interface, and under what conditions shell 
formation occurs. In addition, it is not clear from where the crystal nucleation in the soccer balls starts: in the bulk liquid, at the liquid-air interface or at the micropillars. The size of the crystalline domains is not known, since we have only inspected the surface of the balls and the global packing fraction. It would be interesting to analyse the internal structure of the balls in more detail, e.g. by making straight cuts through the bulk of the balls or by using X-ray techniques.

Finally, in Chapter 7 we investigated the inverse of a liquid drop in air: an air bubble in liquid. We studied the dynamic response of gas bubble entrapped in a cavity on a submerged solid, and calculated the interface shape and resonance frequency of such a bubble when it is perturbed by an acoustic field. We found that the resonance frequency and interface shape depend on two dimensionless parameters: the ratio of the gas stiffness to the surface tension stiffness, and the Ohnesorge number, representing the relative importance of viscous forces. The resonance frequency of such a micropit bubble can accurately be predicted by a potential flow model. To obtain an estimate for the damping of the oscillations, a Stokes flow model is required.

We conclude this thesis by indicating possible directions for future work. We have demonstrated that for macroscopic drops under atmospheric conditions the evaporation rate is governed by the diffusion of vapour in the surrounding air. However, under slightly different conditions, other mechanisms could influence the evaporation rate of a sessile drop, such as the ambient pressure [5], the thermal properties of the liquid and the substrate [6,7], or the transfer rate of molecules across the liquid-air interface $[8,9]$. During the evaporation of a sessile drop all these processes play a role, but the evaporation rate will be determined by the slowest one. Despite the fact that in literature all these different mechanisms have been described, the exact regimes where each mechanism dominates have never been clearly established. Hence, what is still lacking is a description of the complete phase space of an evaporating drop.

An additional complication is that the relative importance of the different evaporation mechanisms will depend on the distance to the contact line. Close to the contact line, the diffusive evaporative flux from the drop surface diverges for contact angles below $90^{\circ}$ [10]. It remains an open question what mechanism regularizes this singularity in the evaporative flux. Several regularizing mechanisms could play a role, such as the Kelvin effect [11], the mean free path of the vapour molecules (see the discussion in Chapter 3), or the presence of a precursor film on complete wetting substrates [12].

In this thesis, we have mainly been concerned with drops with pinned contact lines. An additional complication arises when the contact line recedes during evaporation. For moving contact lines, one has to distinguish between two different cases: partial wetting and complete wetting. For partially wetting substrates, the moving 
contact-line solution by Huh \& Scriven [13] can be superimposed on the evaporationdriven solution. During evaporation of the drop, the contact line will recede with a contact angle close to its equilibrium value, because the flow inside the drying drop does not generate a large enough stress to substantially deform the interface. For complete wetting the situation is totally different: the equilibrium angle is equal to zero and will never be reached. In this case, the tendency of the drop to spread is in competition with the evaporation and the contact angle remains finite. This poses a challenging problem: in the description of a drying drop on a complete wetting substrate the drop shape, internal fluid flow and evaporative flux are all coupled, and the local solutions close to the contact line depend on the global evaporation characteristics. Wetting in the presence of drying is therefore still subject of ongoing work [12, 14-17], and no consensus on the evolution of the drop radius, the apparent contact angle and the width of the contact line region has been reached. We are currently working on a local similarity solution for the contact line region that captures the behaviour of the drying drop in complete wetting on small scales.

Our analytical model of the flow in a drying drop predicts interesting flow structures near the contact line for large contact angles. We hope that our work will stimulate new experiments to measure, e.g. by $\mu$ PIV, the velocity field in an evaporating drop with a contact angle on the scale where the convection rolls occur. It will also be interesting to study how these different flow structures affect the particle deposition. It has been demonstrated that convection rolls in an evaporating drop due to the Marangoni effect may significantly alter the deposition pattern and that this additional circulation could even prevent the coffee-stain effect [2].

In the present work, we have shown that there are various ways to influence the deposition patterns formed by evaporating drops, by controlling the internal fluid flow, the evaporation rate, the contact angle of the drops, the contact-line motion, the structure of the substrate that is used, the particle size and number of particles in the system. However, we have not yet revealed the details of the dynamical process of particle deposition within the drying stains or soccer balls. A crucial factor in the particle deposition is the interaction of the particles with the liquid-air interface and with each other. These interactions can for example be modified by changing the particle shape [18], the salt concentration in the liquid to modify the electrostatic interactions [19], or wetting properties of the particles [20]. Recently, it has been shown that the deposit growth process strongly depends on the particle shape [21]. An interesting direction for future research is to study this final stage of the deposition dynamics in more detail: how does the dynamical process of particle ordering occur in three dimensions? 


\section{References}

[1] W. D. Ristenpart, P. G. Kim, C. Domingues, J. Wan, and H. A. Stone, "Influence of substrate conductivity on circulation reversal in evaporating drops", Phys. Rev. Lett. 99, 234502 (2007).

[2] H. Hu and R. Larson, "Marangoni effect reverses coffee-ring depositions", J. Phys. Chem. B 110, 7090-7094 (2006).

[3] A. G. Marín, H. Gelderblom, D. Lohse, and J. H. Snoeijer, "Order-to-disorder transition in ring-shaped colloidal stains", Phys. Rev. Lett. 107, 085502 (2011).

[4] N. Tsapis, E. Dufresne, S. Sinha, C. Riera, J. Hutchinson, L. Mahadevan, and D. Weitz, "Onset of buckling in drying droplets of colloidal suspensions", Phys. Rev. Lett. 94 (2005).

[5] K. Sefiane, S. K. Wilson, S. David, G. J. Dunn, and B. R. Duffy, "On the effect of the atmosphere on the evaporation of sessile droplets of water", Phys. Fluids 21, 062101 (2009).

[6] S. David, K. Sefiane, and L. Tadrist, "Experimental investigation of the effect of thermal properties of the substrate in the wetting and evaporation of sessile drops", Colloids Surf. A 298, 108-114 (2007).

[7] G. J. Dunn, S. K. Wilson, B. R. Duffy, S. David, and K. Sefiane, "The strong influence of substrate conductivity on droplet evaporation", J. Fluid Mech. 623, 329-351 (2009).

[8] J. P. Burelbach, S. G. Bankoff, and S. H. Davis, "Nonlinear stability of evaporating condensing liquid films", J. Fluid Mech. 195, 463-494 (1988).

[9] N. Murisic and L. Kondic, "On evaporation of sessile drops with moving contact lines”, J. Fluid Mech. 679, 219-246 (2011).

[10] R. D. Deegan, O. Bakajin, T. F. Dupont, G. Huber, S. R. Nagel, and T. A. Witten, "Capillary flow as the cause of ring stains from dried liquid drops", Nature 389, 827-828 (1997).

[11] V. Janecek, B. Andreotti, D. Prazak, T. Barta, and V. S. Nikolayev, "Moving contact line of a volatile fluid", ArXiv:1212.3717 (2013).

[12] J. Eggers and L. M. Pismen, "Nonlocal description of evaporating drops", Phys. Fluids 22, 112101 (2010). 
[13] C. Huh and L. E. Scriven, "Hydrodynamic model of steady movement of a solid/liquid/fluid contact line", J. Colloid Interface Sci. 35, 85-101 (1971).

[14] C. Poulard, O. Benichou, and A. M. Cazabat, "Freely receding evaporating droplets", Langmuir 19, 8828-8834 (2003).

[15] C. Poulard, G. Guéna, A. M. Cazabat, A. Boudaoud, and M. Ben Amar, "Rescaling the dynamics of evaporating drops", Langmuir 21, 8226-8233 (2005).

[16] G. Guéna, C. Poulard, and A. M. Cazabat, "The leading edge of evaporating droplets”, J. Colloid Interface Sci. 312, 164-171 (2007).

[17] G. Berteloot, C. T. Pham, A. Daerr, F. Lequeux, and L. Limat, "Evaporationinduced flow near a contact line: consequences on coating and contact angle", Europhys. Lett. 83, 14003 (2008).

[18] P. J. Yunker, T. Still, M. A. Lohr, and A. G. Yodh, "Suppression of the coffeering effect by shape-dependent capillary interactions", Nature 476, 308-311 (2011).

[19] A. Merlin, J.-B. Salmon, and J. Leng, "Microfluidic-assisted growth of colloidal crystals", Soft Matter 8, 3526-3537 (2012).

[20] T. P. Bigioni, X. M. Lin, T. T. Nguyen, E. I. Corwin, T. A. Witten, and H. M. Jaeger, "Kinetically driven self assembly of highly ordered nanoparticle monolayers", Nat. Mater. 5, 265-270 (2006).

[21] P. J. Yunker, M. A. Lohr, T. Still, A. Borodin, D. Durian, and A. G. Yodh, "Effects of particle shape on growth dynamics at edges of evaporating drops of colloidal suspensions", Phys. Rev. Lett. 110, 035501 (2013). 


\section{Samenvatting}

Als een vloeistofdruppel waarin deeltjes zijn opgelost opdroogt, ontstaat er een karakteristiek patroon. Iedereen kent bijvoorbeeld de donkere kringen die achterblijven op het tafelblad nadat je koffie of thee hebt gemorst. Dit alledaagse verschijnsel is eigenlijk heel opmerkelijk: de koffiedeeltjes, die oorspronkelijk gelijkmatig over de druppel verdeeld waren, hopen zich uiteindelijk allemaal op in een ringvormig patroon. Dit coffee-stain effect komt niet alleen thuis voor, maar ook in allerlei industriële processen. In de coating- en printingindustrie vormen niet-uniform opdrogende druppels van een suspensie een groot probleem. De industrie kan echter ook handig gebruik maken van het coffee-stain effect. De verdamping van druppels met daarin opgeloste colloïdale deeltjes biedt de mogelijkheid kleine structuren te maken op een schaal waarop je de deeltjesdepositie niet meer rechtstreeks kan manipuleren. Om gebruik te kunnen maken van het coffee-stain effect of het juist te voorkomen, is het van belang om te begrijpen hoe deeltjestransport in opdrogende druppels plaatsvindt. Hiertoe is fundamentele kennis nodig over druppelverdamping, de vloeistofstroming in verdampende druppels en het transport van deeltjes.

In dit proefschrift hebben we de fysica van verdampende druppels bestudeerd aan de hand van een eenvoudig modelsysteem: een macroscopische waterdruppel die onder atmosferische conditities verdampt vanaf een oppervlak. In deze druppel losten we kleine colloïdale deeltjes op en bestudeerden de gevormde patronen.

In hoofdstuk 2 hebben we de verdamping van druppels vanaf een superhydrofoob oppervlak bestaande uit koolstofnanovezels bestudeerd. Op dit type oppervlak verplaatst de contactlijn van de druppels zich niet tijdens de verdamping, terwijl de contacthoek van de druppel steeds kleiner wordt. Het bleek dat deze afname van contacthoek en druppelmassa nauwkeurig beschreven kon worden met een diffusiemodel. Hieruit maakten we op dat de verdamping van waterdruppels op dit type oppervlakken quasistatisch en diffusiegedreven is en dat thermische effecten een verwaarloosbare invloed hebben op de verdampingssnelheid.

De verdampingsflux vanaf het oppervlak van een opdrogende druppel leidt tot een vloeistofstroming binnenin de druppel. Hoe deze stroming er precies uitziet hebben we bestudeerd in hoofdstuk 3. We hebben laten zien dat er gelijkvormigheidsoplos- 
singen bestaan voor de vloeistofstroming dichtbij de contactlijn van de druppel. Er zijn drie mechanismes die stroming kunnen genereren. Allereerst natuurlijk de verdampingsflux van het druppeloppervlak zelf. De verdamping zorgt er echter ook voor dat het grensvlak tussen de druppel en de omringende lucht naar beneden beweegt. Deze grensvlakbeweging werkt als een dichtklappend scharnier: het scharnier duwt de vloeistof van de contactlijn weg. Ten derde is er een zogenaamde "eigenmodus"bijdrage aan de vloeistofstroming bij de contactlijn. Deze eigenmodusoplossing voldoet aan de homogene randvoorwaarden en kan resulteren in viskeuze wervelingen in de stroming. Het is afhankelijk van de contacthoek van de druppel welke van deze drie stromingsmechanismen het sterkst is. Voor contacthoeken lager dan een kritische waarde van $133.4^{\circ}$ is de verdampingsgedreven oplossing het sterkst, terwijl voor grotere hoeken de eigenmodusoplossing de stroming domineert.

In hoofdstuk 4 hebben we gekeken naar wat er gebeurt als een druppel niet vanaf een vast, maar vanaf een vloeibaar oppervlak verdampt. In dat geval is het oppervlak niet alleen vervormbaar, maar genereert de verdamping van de druppel ook een stroming in het vloeibare oppervlak. De contactlijn van de druppel zit niet langer vast aan het substraat, maar beweegt naar binnen toe. Deze terugtrekkende contactlijn geeft een bijdrage aan de stroming in de druppel, evenals de verdampingsflux van het oppervlak en de eigenmodusoplossing. De eigenmodusoplossing blijkt echter voor alle contacthoeken subdominant te zijn. Voor contacthoeken tussen de druppel en het horizontale vloeibare oppervlak die kleiner zijn dan $90^{\circ}$ domineert de bijdrage van de terugtrekkende contactlijn de stroming; voor grotere contacthoeken is de verdampingsflux dominant. Hoeveel stroming de verdampende druppel in het vloeibare oppervlak genereert is afhankelijk van de verhouding in de viscositeit tussen de twee vloeistoffen. Afhankelijk van deze viscositeitsverhouding vindt men dezelfde soort terugstromingsstructuren als beschreven in hoofdstuk 3, maar dan in beide vloeistoffases.

Hoe de vloeistofstroming in een verdampende druppel er precies uitziet bepaalt ook hoe het transport van in de druppel opgeloste deeltjes plaatsvindt. Als de contactlijn van de druppel vastzit aan het oppervlak ontstaan de kringvormige patronen die we kennen van opdrogende koffie- en theedruppels. In hoofdstuk 5 hebben we de dynamica van deze kringvorming bestudeerd. We hebben aangetoond dat de structuur van de gevormde kringen afhankelijk is van de competitie tussen deeltjesdiffusie en -convectie tijdens het opdrogen van de druppel. Tijdens de laatste fase van de druppelverdamping neemt de snelheid waarmee deeltjes naar de contactlijn bewegen enorm toe. Wanneer deeltjes met een hoge snelheid bij de contactlijn aankomen hebben ze geen tijd om zich te rangschikken in een geordend patroon en ontstaat een wanordelijke structuur. Deeltjes die vroeg in het verdampingsproces aankomen, met 
lage snelheid, hebben daarentegen voldoende tijd om zich door middel van Brownse beweging te ordenen. In dat geval ontstaat een geordende, kristallijne structuur. De buitenste, bij lage snelheid gevormde delen van de deeltjeskring zijn dus geordend, terwijl verder naar binnen een wanordelijk patroon te zien is. Het gevormde patroon kan worden beïnvloed door de verdampingssnelheid van de druppel en de deeltjesgrootte, en daarmee de tijd die deeltjes hebben om zich te ordenen, te controlen.

Wanneer de contactlijn van een opdrogende druppel niet vastgepind zit maar vrij kan bewegen, kunnen zich heel andere patronen van deeltjes vormen. In hoofdstuk 6 lieten we een druppel verdampen vanaf een speciaal soort superhydrofoob substraat, bestaande uit kleine pilaartjes. Tijdens de verdamping bleef de druppel bovenop deze pilaartjes zitten, zoals een fakir op een spijkerbed, terwijl de contactlijn zich naar binnen bewoog. Aan het eind van het verdampingsproces bleken alle colloïdale deeltjes die in de druppel zaten zich te hebben gegroepeerd in een balletje met een zeer grote dichtheid. De stapeling van deeltjes in deze balletjes was dan ook bijna perfect. Opnieuw bleek de rangschikking van de deeltjes het resultaat te zijn van een competitie tussen deeltjesdiffusie en -convectie. De convectieve beweging is in dit geval afkomstig van de inwaartse beweging van het druppeloppervlak, dat de deeltjes naar binnen duwt. Door de verdampingssnelheid van de druppel en het aantal deeltjes in de druppel te controleren kan men de rangschikking van deeltjes in het balletje beïnvloeden. De in hoofdstuk 6 beschreven methode kan dus gebruikt worden om minuscule driedimensionale geordende structuren te maken met verdampende druppels.

In hoofdstuk 7 hebben we gekeken naar het omgekeerde van een druppel in lucht: een bel in vloeistof. We bestudeerden bellen die zijn ingevangen in een holte op een oppervlak dat is ondergedompeld in een vloeistof en berekenden de reactie van zo'n bel op een drukgolf. We hebben een model gemaakt om de vorm van het beloppervlak en de resonantiefrequentie van de bel te bepalen. Deze resonantiefrequentie en de vorm van het oppervlak bleken te worden bepaald door twee dimensieloze parameters: de verhouding tussen de stijfheid van het gas in de bel en de stijfheid van het beloppervlak en het Ohnesorgegetal, dat een maat is voor de invloed van viskeuze krachten. De resonantiefrequentie van de bel kon nauwkeurig bepaald worden met een potentiaalstromingsmodel. Voor het berekenen van de demping van de beltrillingen moest een Stokesstromingsmodel gebruikt worden. 


\title{
Acknowledgements
}

\author{
"Supposing a tree fell down \\ Pooh, when we were underneath \\ it?" said Piglet. \\ "Supposing it didn't," said Pooh \\ after careful thought.
}

A. A. Milne, The house at the

Pooh corner.

After four years of work, there are many people I would like to thank for their contributions to this thesis, their support, advice and encouragement. First of all, I was very lucky to have the best PhD-advisors one could wish for: Detlef Lohse and Jacco Snoeijer. Detlef, thanks for all the confidence and freedom you gave me right from the start of my PhD project. Initially, the fact that I had no clearly defined project made me feel a little nervous, but looking back I am glad I always had the opportunity to dive into an interesting topic and change directions whenever I wanted to. I have learned a lot from the way you practise science and from the great working environment you have created within the PoF group. It was great that you gave me the opportunity to meet with scientists around the world and visit many conferences and summer schools. I am amazed and very grateful that, even though you are extremely busy, you always find time to discuss, give feedback on papers and talks and provide support. I am looking forward to continue our collaboration in the future.

When I arrived at PoF there were many things I had to learn and get used to. I cannot imagine a better guide into the world of science than Jacco Snoeijer. Jacco, you have taught me everything I need to know to survive (at least scientifically); from the very basic physical principles, the use of scaling arguments and self-similarity, to how to give talks and write papers, and how to deal with scientists (which is not always trivial). I have good memories of all the nice things we have done together, such as the trip to Bruxelles, the "Huis- tuin- en keukenfysica" Science café, dealing with journalists from de NRC and de Telegraaf, the APS-DFD in Baltimore, the 
speakers contest, writing papers and throwing dictionaries, and of course the nice dinners and drinks with Daniëlle, Leon and Lucie. Apart from that, you were always there to help me or give me confidence whenever I needed it. Many thanks for all of that and I am very happy that we can continue our joint work!

Besides Detlef and Jacco, there are many other people with whom I had the pleasure to collaborate and discuss, and who contributed considerably to this thesis. Leen van Wijngaarden, thank your very much for all your advice and help. It was great that you were always available to discuss about any fluid-mechanics problem I encountered. I have very good memories of my visit to you and Willy in Les Contamines Montjoie in July 2012. Álvaro Gomez Marín, your contribution to my thesis has been tremendous. From the moment we started to collaborate my $\mathrm{PhD}$ work really came into the right "flow". It is fair to say that without your great experimental skills there would have been no Chapter 2, 5 and 6 in this thesis. Howard Stone, I am glad that our discussions in Les Houches led to a nice joint paper, which forms the basis for Chapter 4 of this thesis. Andrea Prosperetti, it was a great pleasure to work with you. Thank you very much for all your ideas and suggestions, advice and help, which resulted in Chapter 7 of this thesis. Hrudya Nair, Arturo Susarray-Arce and Aaldert Zijlstra, thank you for your contributions to Chapter 2, 6, and 7, respectively. Oscar Bloemen, I enjoyed assisting you with your Master's project. Thank you for your contributions to Chapter 3. I would like to acknowledge Dirk Aarts, Christophe Clanet, Pierre Colinet, Jens Eggers, Arie van Houselt, David Quéré, Paul Steen, Fons van de Ven and Harold Zandvliet for valuable discussions about the work presented in this thesis. Jens, thank you for inviting me for a stay in Bristol! Many thanks to Ramin Badie, Jos Benschop, Hans Hilgenkamp, Frans van de Vosse and Stephen Wilson for their interest in my work and for being a member of my $\mathrm{PhD}$ committee. Frans, I am happy that we are still in contact. It is also thanks to you that I ended up in this nice group for my $\mathrm{PhD}$. Ramin and Jos, I am very much looking forward to our joint future work at ASML.

I am sure that the PoF group would not be as efficient and productive as it is now without Bas Benschop, Gert-Wim Bruggert, Martin Bos and Joanita Leferink. Bas, thanks to you I never had to worry about computer issues, which saved me from lots of trouble and frustration. Joanita, thanks for all your help with organizing the group seminars, the primary school day, the ASML workshop, etc.; I would not have managed without you! Devaraj van der Meer, Ivo Peters and Laura Stricker, I enjoyed teaching the course Heat \& Mass Transfer together; I have learned a lot from all of you. Pascal Sleutel, it was fun to organize the ASML workshop with you, and I am glad we will continue collaborating in the future.

The PoF group is a very nice and social group, which helped me to feel at home in 
Enschede. I want to thank everyone for the nice dinners, evenings, outings, summer schools and conferences we have had together. In particular, I would like to mention Joost, Tess, Oscar, Rianne, Claas Willem, Laura, Álvaro, Antonin, Henri, Koen, Sander and Arjan. A special thanks goes to my office mates from the Left-Handed Vegetarian Office. Oscar and Joost, it was always great fun to share an office with you! A special thanks also to the Les Houches team -Claas Willem, Sander, Wilco and Tak Shing- for the best summer school ever (I am still amazed how much stuff fits into a Kia Picanto)!

I am very fortunate to have wonderful friends, on whom I could always count. Tess, Joost, Rianne and Claas Willem, thanks for always being there for a chat, gezelligheid and support. I will surely miss living close to you! A special thanks goes to Koen; we have had a nice time together, for which I am very grateful. I thank the ladies from the G.U.P.P.I.E.S. (Sarah, I enjoyed our muddy mountain bike rides very much!) and all the people from Z.V.P. Piranha for four nice years of waterpolo. Rik, it is a comforting feeling to have your "dad" looking after you, thanks for always being there! I would like to thank De Tackûhlaars, and in particular Jasper, Petra and of course Bram, for their friendship and all the fun we have had together. Bram, I am very grateful for all the support you have given me during the first year of my $\mathrm{PhD}$. Xanthe, we have been friends for 17 years now, and I hope to add at least another 17! Thank you very much for designing the cover of this thesis. Sjoerd, Anne, Miriam, Sten, Emmy, Jarno, Elise, Maarten and Arjan, alias The Movers, the (holi)days we spend together moving, hiking, canoeing, mountain biking, cycling through not-sosunny Andalusia, camping, loosing games against Sten, cooking and enjoying a drink on a sunny terrace are always fantastic.

Miriam and Anne, it is great to have you next to me as my paranimfen during the defence. You have given me so much support during my entire $\mathrm{PhD}$ period that I can only hope I can do the same for you. Opa, I feel honoured that you are present at my defence. Jaap, thanks for being such a great brother! Jaap, Onno, Grietina, Opa, Pap and Mam, your sense of humour and the way you can put things into perspective really helps me to remember the first sentences of these Acknowledgements. Pap en Mam, ik kan nu in allerlei clichés vervallen, maar het volgende dekt de lading toch het best: bedankt voor alles!!

Hanneke Gelderblom

Enschede, February 2013 


\section{About the author}

Hanneke Gelderblom was born on September 30th 1985 in Eindhoven. After one year, she moved with her parents to the village of Nuenen, where she spent the next 17 years of her life. She obtained her Gymnasium diploma at "het Strabrecht College" in Geldrop in 2003. Then, she moved to Eindhoven where she studied Biomedical Engineering at Eindhoven University of Technology. She participated in many student activities, including the organization of a study tour to China in March 2006. In 2007 she spent four months in New Zealand for an internship at the Auckland Bioengineering Institute with Dr. Martyn Nash and Prof. Peter Hunter, where she developed a finite element model to study the mechanics of the human left ventricle. For her Master's thesis, Hanneke worked on the analytical and experimental characterization of a thermal sensor for coronary flow assessment, in the group of Prof. Frans van de Vosse. She obtained her Master's diploma in 2009 (cum laude). In May 2009 she moved to Enschede to start her PhD research in the Physics of Fluids group with Prof. Detlef Lohse and Dr. Jacco Snoeijer at the University of Twente.

Apart from doing research, Hanneke likes to spend time with her friends, on a bike, in the mountains, on holiday (or any arbitrary combination of these four), in the kitchen, behind the easel and in the swimming pool. 
